\title{
Syndromic surveillance : made in Europe
}

Citation for published version (APA):

Ziemann, A. (2015). Syndromic surveillance : made in Europe. [Doctoral Thesis, Maastricht University]. Maastricht University. https://doi.org/10.26481/dis.20151207az

Document status and date:

Published: 01/01/2015

DOI:

10.26481/dis.20151207az

Document Version:

Publisher's PDF, also known as Version of record

\section{Please check the document version of this publication:}

- A submitted manuscript is the version of the article upon submission and before peer-review. There can be important differences between the submitted version and the official published version of record.

People interested in the research are advised to contact the author for the final version of the publication, or visit the DOI to the publisher's website.

- The final author version and the galley proof are versions of the publication after peer review.

- The final published version features the final layout of the paper including the volume, issue and page numbers.

Link to publication

\footnotetext{
General rights rights.

- You may freely distribute the URL identifying the publication in the public portal. please follow below link for the End User Agreement:

www.umlib.nl/taverne-license

Take down policy

If you believe that this document breaches copyright please contact us at:

repository@maastrichtuniversity.nl

providing details and we will investigate your claim.
}

Copyright and moral rights for the publications made accessible in the public portal are retained by the authors and/or other copyright owners and it is a condition of accessing publications that users recognise and abide by the legal requirements associated with these

- Users may download and print one copy of any publication from the public portal for the purpose of private study or research.

- You may not further distribute the material or use it for any profit-making activity or commercial gain

If the publication is distributed under the terms of Article $25 \mathrm{fa}$ of the Dutch Copyright Act, indicated by the "Taverne" license above, 


\section{SYNDROMIC SURVEILLANCE}

\section{MAde in Europe}

Alexandra Ziemann 
Syndromic Surveillance - Made in Europe

(C) Alexandra Ziemann, 2015

All rights reserved. No part of this publication may be reproduced without permission of the copyright holder.

ISBN 978-3-00-051210-0

Printed by: Druckerei Wellmann (Bremen, Germany)

Cover design by: Alexandra Ziemann, Christoph Ziemann \& Mieke Ziemann 


\section{SYNDROMIC SURVEILLANCE}

\section{MADE IN EUROPE}

\section{Dissertation}

to obtain the degree of Doctor at the Maastricht University, on the authority of the Rector Magnificus,

Prof. dr. L. L. G. Soete,

in accordance with the decision of the Board of Deans,

to be defended in public

on Monday, 7 December 2015, at 14:00 hours

by

Alexandra Ziemann 


\section{Supervisors}

Prof. dr. Helmut Brand

Prof. dr. Thomas Krafft

\section{Assessment Committee}

Prof. dr. Jouke van der Zee (chairman)

Assoc. prof. dr. Katarzyna Czabanowska

Dr. Anette Hulth (Public Health Agency of Sweden, Sweden)

Prof. dr. Thomas Kistemann (University of Bonn, Germany)

Prof. dr. David Townend

The research for this dissertation was performed at the Department of International Health, School for Public Health and Primary Care (CAPHRI), Faculty of Health, Medicine and Life Sciences, Maastricht University, which participates in the Netherlands School for Primary Care Research (CaRe), acknowledged by the Royal Dutch Academy of Science (KNAW).

Financial support for the actions "European Emergency Data-based System for Information on, Detection and Analysis of Risks and Threats to Health" (SIDARTHa, Grant Agreement No. 2007208) and "Syndromic Surveillance Survey, Assessment towards Guidelines for Europe" (Triple S-AGE, Grant Agreement No. 20091112) were provided by the European Commission in the framework of the Health Programme. 


\section{Contents}

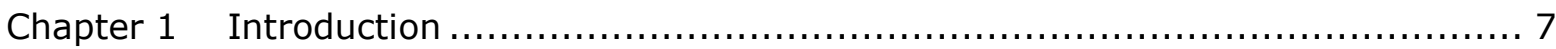

Chapter 2 A new (European) Syndromic Surveillance Definition .......................19

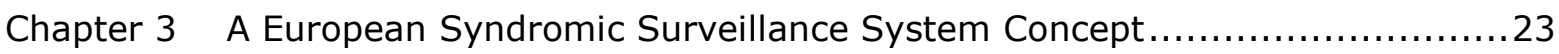

Chapter 4 Syndromic Surveillance Supporting Local Influenza Surveillance ............43

Chapter 5 The Added Value of Syndromic Surveillance for Implementing the International Health Regulations (2005) .................................. 57

Chapter 6 Success Factors of European Syndromic Surveillance Systems............. 81

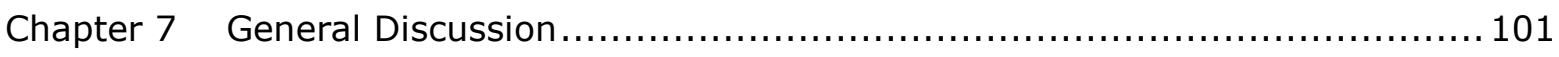

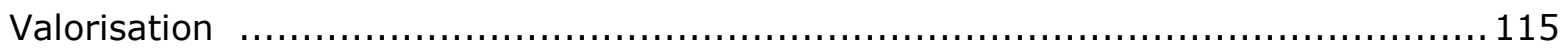

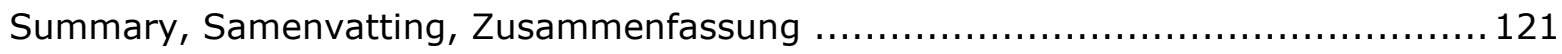

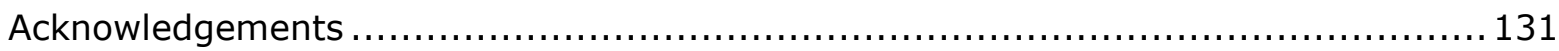

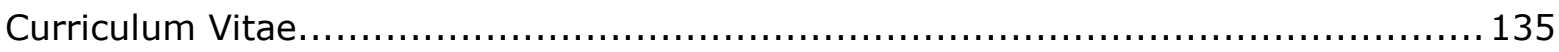



CHAPTER 1

Introduction 


\section{Syndromic surveillance}

Our globalised world faces new, unprecedented and diverse challenges for public health. These challenges are resulting from new emerging diseases, extreme environmental events, natural disasters, or man-made threats. They call for quick decisions and actions, also across borders. Such decisions can only be taken based on rapid information about the public health effects of events. Until now, traditional public health surveillance was focusing on specific pre-defined diseases or agents causing certain diseases. This approach cannot cope with diverse, unknown, or unexpected public health threats. In response, the World Health Organization (WHO) revised their International Health Regulations in 2005 (IHR (2005)) from focusing on specific diseases to an all-hazard approach (1). The revised regulations also request the WHO member states to adjust their surveillance capacities. Member states now need to monitor different health threats and to immediately report the results. The approach of syndromic surveillance can help countries to meet the new public health challenges and the new surveillance requirements.

Syndromic surveillance aims at augmenting traditional public health surveillance systems with (near) real-time information on the public health impact of events (2). In order to gain a head start, syndromic surveillance analyses existing, possibly electronic data that usually were not collected for surveillance purposes. These data come from non-clinical sources such as calls to telephone helplines or web searches (3). Or they come from clinical sources such as patient records from primary and emergency care or veterinary records.

Syndromic surveillance was primarily developed in the United States of America (USA) as one answer to the threat of bioterrorism after the 2001 terrorist attacks (4). Several syndromic surveillance systems were developed for the rapid detection and follow up of the impact of bioterrorist attacks on public health. Fortunately, they had not to be used for that purpose often (5). Instead, syndromic surveillance evolved to be applied for other purposes, as Paterson and colleagues showed (6). First, the focus changed to rapid information about the onset and development of seasonal infectious diseases, mainly influenza. Later, syndromic surveillance was used for situational awareness during different events associated with public health risks. Such events are as diverse as the 2012 Olympic Games in London, hurricane Katrina in the USA in 2005, the 2010 volcanic ash plume in Europe, the influx of refugees to Italy since 2011 or the recent financial crisis in Greece (7-11). In Europe, the first systems were established in England in 2001 for augmenting seasonal influenza surveillance and in France in the aftermath of the 2003 heatwave $(12,13)$. In 2012, there were about 60 active, pilot, planned or terminated syndromic surveillance systems in more than 15 European countries $(3,14)$.

Syndromic surveillance is still a relatively new approach and there is still much doubt about its added value (15-17). Timeliness in terms of earlier case detection and/or reporting results is considered the main strength of the approach $(18,19)$. The provision of information at times when no information is available from other (traditional) surveillance systems can be considered another advantage $(8,20,21)$. Also the flexible application for different health threats is a strength of syndromic surveillance $(6,22)$. The major weakness is seen in the lack of specificity of the output of syndromic surveillance systems. They analyse existing data sources and signs, symptoms and proxy measures that are not indicating one specific health effect or disease. This non-specificity can lead to false alerts or not detecting events (15). By assessing the performance of the various 
syndromic surveillance applications, we can now compile more conclusive evidence on the usefulness of the approach.

\section{Made in Europe}

\section{A unique context...}

Europe, in this thesis usually referring to the European Union (EU), is a unique structure. It is not like a state although it has many state characteristics such as legislative, executive and judicative bodies. It is a sphere of common history and values, close relations but also great diversity. EU interventions are following certain principles to balance European integration and European diversity (23). These principles were originally established to define the competences of the EU. The basic principle of European integration or harmonisation refers to creating European standards which replace differing national standards. The principle of subsidiarity is the opposite of European harmonisation efforts. It entails that tasks which are better performed by member states should also be performed by member states instead of by the EU. The principle of mutual recognition refers to member states respecting regulations, goods, services or people of other member states. This has the advantage that the EU does not have to regulate every detail. Although these principles are usually applied to legal questions, they might also be applicable to assess the European fit of any strategy or action that has to perform well in the European context.

\section{...for fighting health threats}

Greer and colleagues have summarised the role of the European Union regarding health issues as "somewhat paradoxical" (23, p.xi). While having agreed on health being a common core value (24), member states keep a strong own position regarding health issues (23). The EU has a limited direct mandate for health through Article 168 of the Treaty on the Functioning of the European Union. But, the impact of the Union is largely indirect via the coordination of social security, environmental protection and occupational safety but also via regulations on the internal market or free movement, for example. Often, crises had an impact on health policy change at the European level. One examples is the 2003 SARS outbreak which led to the establishment of the European Centre for Disease Prevention and Control (23). As health threats do not stop at borders, the EU focuses more on harmonisation in the area of health security. A recent example is the decision on cross-border health threats (25). The decision aims at implementing the IHR (2005) in Europe and aims at improving coordination and exchange between member states and with the European Commission. It especially also focuses on monitoring and early warning of health threats.

Member states frequently join forces to conquer similar health problems or they learn from states who have already experiences in tackling them. The European Commission supports such activities by co-funding actions in the framework of the Health Programme with the aim: "[...]to complement national policies of the Member States with a European added-value [...], [produce] results [that] should be able to be applied in other countries and regions across Europe [...], [and to] support developments at European level in the field of health." (26, p. 3, 17). The EU principles of harmonisation, mutual recognition and subsidiarity are also reflected in Health Programme actions. In this context, Brand 
discussed a dichotomy of actions characterised as 'public health in Europe' and those characterised as 'European public health' (27). 'Public health in Europe' refers to more reactive or retrospective actions regarding health issues in several European countries. The more proactive 'European' approach covers actions such as defining generic European frameworks, adopting common policies across Europe, or centralising actions at European level. Health Programme actions are often characterised by the setup of a network and knowledge exchange between member states, going more in the direction of 'public health in Europe'. Further, actions also focus on harmonisation efforts, defining common standards or strategies, which rather fall under 'European public health'.

There are various Health Programme actions on health security and surveillance $(28,29)$. Between 2007 and 2013, there were two actions focussing on syndromic surveillance. The first action "European Emergency Data-based System for Information on, Detection and Analysis of Risks and Threats to Health" (SIDARTHa, Grant Agreement No. 2007208) intended to develop a European concept for syndromic surveillance based on emergency care data at the subnational level (30). The second action "Syndromic Surveillance Survey, Assessment towards Guidelines for Europe" (Triple S-AGE, Grant Agreement No. 20091112) aimed at harmonising syndromic surveillance across Europe and providing European guidelines to foster implementation of syndromic surveillance (31). These two Health Programme actions provided a unique framework for studying the interactions between the European context and the new concept of syndromic surveillance. They offered the chance to assess if and how the results of the two actions went with the European principles of harmonisation, mutual recognition and subsidiarity.

\section{...for generating new knowledge}

Europe has repeatedly been coined a "natural laboratory" for learning about health (3234). The diversity in Europe somewhat limits the integration efforts, but on the other hand it provides a unique context to gain new knowledge by analysing the differences between countries (33). But, how does this cross-country learning process in Europe actually look like? In general, two different rationales for cross-country learning can be differentiated.

Marmor and colleagues have described the process of cross-country learning from the perspective of countries learning from others (35). Here, new knowledge is mainly generated in and for the individual countries. According to Marmor et al., the learning process takes three different forms: learning about, learning why and learning from. 'Learning about' refers to an often descriptive understanding of similarities and differences. 'Learning why' aims at explaining the way a certain aspect has taken the form it did. 'Learning from' refers to the transfer of a certain aspect from one country or a group of countries to another.

Cross-country learning can also be described from a broader perspective of gaining new knowledge from comparing differences between several countries. A good example for this form of cross-country learning in the European context is the work of the WHO European Observatory on Health Systems and Policies $(36,37)$. Here, new knowledge is generated for one or several other countries, based on benchmarking and the identification of good practices. Such cross-country comparisons have helped reforming health policies, regarding cancer services in England, for example (38). However, there are obstacles for good quality country comparisons. They lie in the limited comparability 
of the complex contexts in the different countries, limited data availability and diverse multidisciplinary comparative approaches and methods $(38,39)$. Despite various initiatives, the field of international comparisons between countries in the area of health is still in the development phase (38).

Based on the rationales of cross-country learning and the characteristics of the European context described above, a generic framework for European cross-country learning can be derived (figure 1). The framework is intended to explain how the European context adds value for generating new knowledge on a certain topic. Three dimensions of learning can be differentiated with the European context playing an increasing role from the first to the third dimension. In the first dimension, Europe provides a context in which countries can learn from each other to gain new knowledge for their own situation, referred to as 'Learning in Europe'. In the second dimension, knowledge for Europe is compiled by harmonisation, standardisation, generalisation or identification of a common denominator of a topic. This can be referred to as 'Learning for Europe'. Here, the EU principles as described above should to be taken into account in order for the knowledge to fit into the European context. The third dimension is focusing on 'learning from Europe'. New knowledge on a topic is generated for common use in Europe (and beyond), based on a structured analysis of the differences in Europe. This could be based on cross-country comparisons, for example. The dimensions of 'Learning in Europe' and 'Learning for Europe' can also be linked to Brand's argumentation of 'Public Health in Europe' and 'European Public Health' respectively. In this thesis, the framework was applied to analyse the added value of the European context to learn about syndromic surveillance.

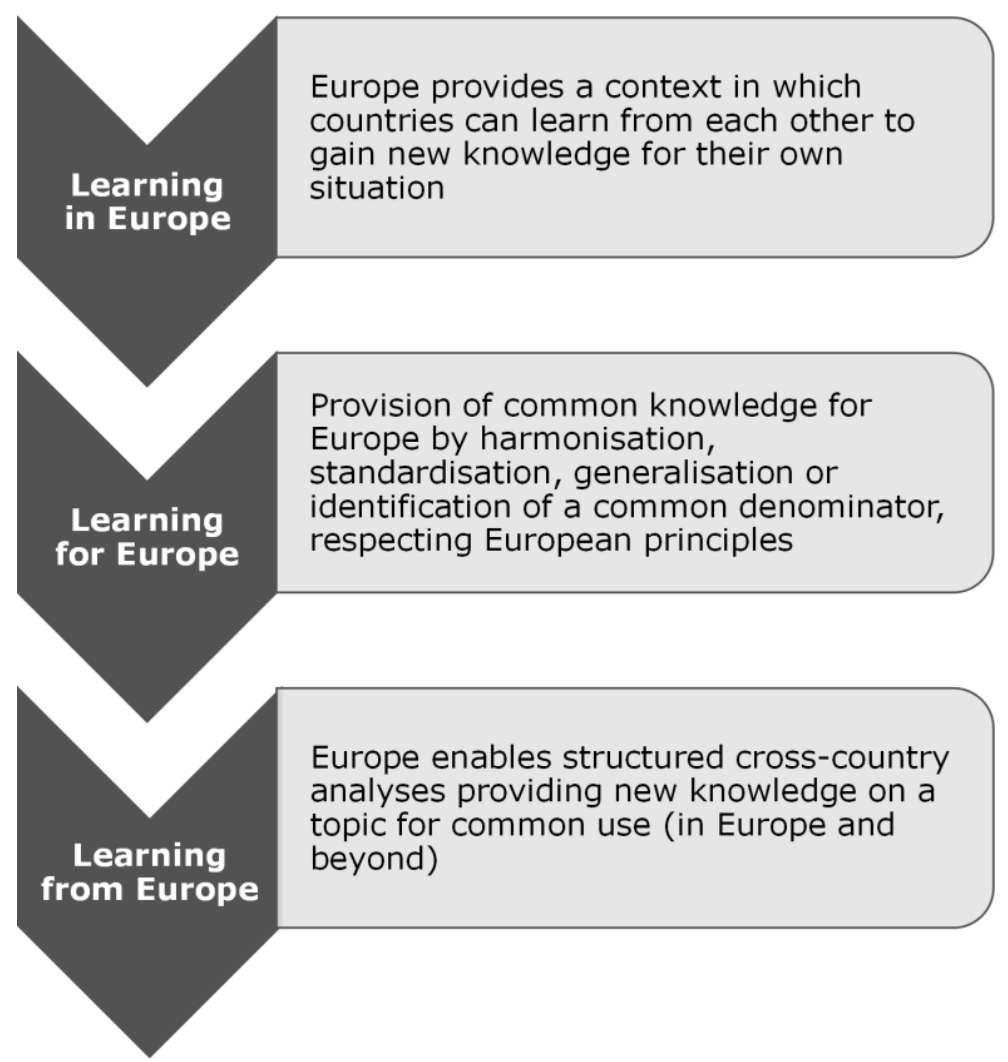

Figure 1: Conceptual framework for cross-country learning in Europe (own concept) 


\section{Aims and outline of the thesis}

The thesis aimed at analysing

1) the added value of syndromic surveillance for improving Europe's surveillance capacity, and

2) the added value of the European context to gain new knowledge about syndromic surveillance.

For the first aim, the thesis explored how a specific European syndromic surveillance system should look like by respecting the European principles of harmonisation, mutual recognition and subsidiarity. Secondly, the usefulness of syndromic surveillance for Europe was explored by assessing the major strengths and weakness of syndromic surveillance: timeliness, flexibility, additional information provision, and non-specificity. Finally, it was assessed how the application of syndromic surveillance can foster Europe's capacity to meet the new IHR (2005) surveillance core capacity requirements (figure 2). For the second aim, the thesis analysed how the European context adds value to learning about syndromic surveillance according to the framework presented in figure 1 . The focus of this analysis was on a cross-country comparison for identifying success factors of syndromic surveillance systems.

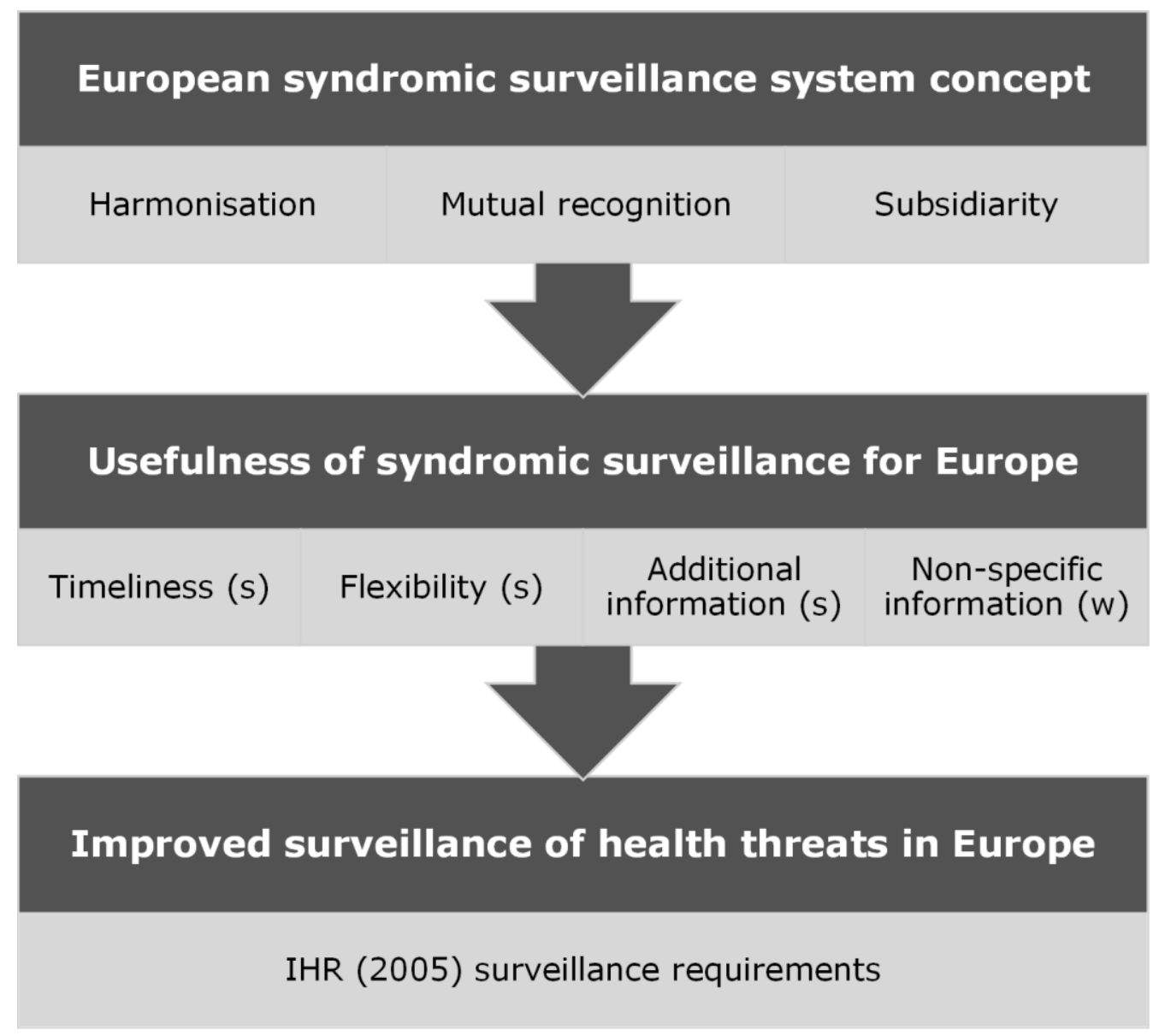

Figure 2: Framework for analysing the added value of syndromic surveillance for Europe (own concept) $\operatorname{IHR}(2005)=$ International Health Regulations (2005) s = strength, $w=$ weakness 


\section{Research questions}

Specifically, the thesis aimed at answering the following research questions (with reference to chapters in this thesis):

1. How can a specific European syndromic surveillance concept be defined?

1.1. How can syndromic surveillance in Europe be defined (chapter 2)?

1.2. How can a European syndromic surveillance system be defined (chapter 3, 5)?

2. How useful is syndromic surveillance for Europe, in terms of monitoring

2.1. gastrointestinal outbreaks (chapter 3 ),

2.2. seasonal influenza (chapters 4,6 ),

2.3. other potentially public health threatening events (chapters 5,6$)$ ?

3. How can syndromic surveillance support the implementation of the IHR (2005) core surveillance capacity requirements in Europe (chapter 5$)$ ?

4. What is the added value of a European cross-country comparison for identifying success factors of syndromic surveillance systems (chapter 6$)$ ?

\section{Research design}

The research questions were answered in several studies which can best be described as following a pragmatic mixed methods research design. Question 1 was answered applying consensus methods. Both questions 1.1 and 1.2 were answered using an expert-panel. The expert panel for question 1.2 was informed by the results of a semi-structured survey and a literature review. Questions 2.1 and 2.2 were answered by applying quantitative analysis methods in a case study design using secondary data from emergency care. For questions 2.3 and 3, a narrative review was applied based on mixed data collected from the literature. Question 4 was answered applying the mixed methods approach Qualitative Comparative Analysis. The study analysed mixed data collected from the literature and from country visits.

The two Health Programme actions SIDARTHa and Triple S-AGE provided a unique framework for studying the research questions. The author had a leading role in both actions and the research presented in this thesis was embedded in the actions. Her research was only possible through the access to data, cases and the network of the partners of these two actions.

\section{Thesis outline}

The thesis has seven chapters. This chapter 1 introduces the basic concepts of syndromic surveillance, the unique European context and cross-country learning. It presents the aims and conceptual frameworks of the thesis, the research questions and the research design. Chapter 2 introduces a new European definition for syndromic surveillance. Chapter 3 describes the development of a concept for a European syndromic surveillance system, including a feasibility study for gastrointestinal outbreak detection. Chapter 4 assesses the application of the European syndromic surveillance concept for supporting local influenza surveillance in one European country. Chapter 5 analyses the potential of syndromic surveillance for situational awareness during different events in order to support the implementation of the IHR (2005) in Europe. It also presents a proposal for the European setup of the syndromic surveillance concept presented in chapter 3 . 
Chapter 6 identifies success factors of syndromic surveillance systems by comparing syndromic surveillance systems from different countries in Europe using Qualitative Comparative Analysis. It also provides further evidence on the usefulness of syndromic surveillance for seasonal influenza surveillance and situational awareness. Chapter 7 synthesises the results in a general discussion regarding the aims of this thesis, discusses limitations and draws conclusions on the practical relevance of the findings and for future research. 


\section{References}

1. World Health Organization. International Health Regulations (2005). Geneva: World Health Organization; 2008.

2. Triple $S$ Project. Assessment of syndromic surveillance in Europe. Lancet. 2011;378(9806):1833-4.

3. Ziemann A, Krafft T. Guidelines for Assessment of Data Sources; 2013 [cited 26 June 2015]. Available from: http://www.syndromicsurveillance.eu/triples_guidelines_datasources.pdf

4. Centers for Disease Control and Prevention. Syndromic surveillance for bioterrorism following the attacks on the World Trade Center--New York City, 2001. MMWR Morb Mortal Wkly Rep. 2002;51 Spec No:13-5.

5. Bravata DM, McDonald KM, Smith WM, Rydzak C, Szeto H, Buckeridge DL, et al. Systematic review: surveillance systems for early detection of bioterrorism-related diseases. Ann Intern Med. 2004;140(11):910-22.

6. Paterson BJ, Durrheim DN. The remarkable adaptability of syndromic surveillance to meet public health needs. J Epidemiol Glob Health. 2013;3(1):41-7.

7. Elliot AJ, Morbey RA, Hughes HE, Harcourt SE, Smith S, Loveridge $P$, et al. Syndromic surveillance - a public health legacy of the London 2012 Olympic and Paralympic Games. Public Health. 2013;127(8):777-81.

8. Murray KO, Kilborn C, DesVignes-Kendrick M, Koers E, Page V, Selwyn BJ, et al. Emerging disease syndromic surveillance for Hurricane Katrina evacuees seeking shelter in Houston's Astrodome and Reliant Park Complex. Public Health Rep. 2009;124(3):364-71.

9. Brand $H$, Krafft $T$. The Icelandic ash cloud and other erupting health threats: what role for syndromic surveillance? Eur J Public Health. 2010;20(4):367-8.

10. Napoli C, Riccardo F, Declich S, Dente MG, Pompa MG, Rizzo C, et al. An early warning system based on syndromic surveillance to detect potential health emergencies among migrants: results of a two-year experience in Italy. Int J Environ Res Public Health. 2014;11(8):8529-41.

11. Economou M, Madianos M, Peppou LE, Patelakis A, Stefanis CN. Major depression in the Era of economic crisis: A replication of a cross-sectional study across Greece. J Affect Disord. 2013;145(3):308-14.

12. Doroshenko A, Cooper D, Smith G, Gerard E, Chinemana F, Verlander N, et al. Evaluation of syndromic surveillance based on National Health Service Direct derived data--England and Wales. MMWR Morb Mortal Wkly Rep. 2005;54 Suppl:117-22.

13. Josseran L, Nicolau J, Caillere N, Astagneau P, Brucker G. Syndromic surveillance based on emergency department activity and crude mortality: two examples. Euro Surveill. 2006;11(12):225-9.

14. Conti S, Kanieff M, Rago G, on behalf of the Triple-S project. Inventory of Syndromic Surveillance Systems in Europe; 2012 [cited 26 June 2015]. Available from: http://www.syndromicsurveillance.eu/images/stories/Final_material/tripleS_country_visits.pdf 
15. Kaydos-Daniels SC, Rojas Smith L, Farris TR. Biosurveillance in outbreak investigations. Biosecur Bioterror. 2013;11(1):20-8.

16. Koopmans M. Surveillance strategy for early detection of unusual infectious disease events. Curr Opin Virol. 2013;3(2):185-91.

17. Morse SS. Public health surveillance and infectious disease detection. Biosecur Bioterror. 2012;10(1):6-16.

18. Buehler JW, Hopkins RS, Overhage JM, Sosin DM, Tong V. Framework for evaluating public health surveillance systems for early detection of outbreaks: recommendations from the CDC Working Group. MMWR Recomm Rep. 2004;53(RR5):1-11.

19. Dailey L, Watkins RE, Plant AJ. Timeliness of data sources used for influenza surveillance. J Am Med Inform Assoc. 2007;14(5):626-31.

20. Elliot AJ, Singh N, Loveridge P, Harcourt S, Smith S, Pnaiser R, et al. Syndromic surveillance to assess the potential public health impact of the Icelandic volcanic ash plume across the United Kingdom, April 2010. Euro Surveill. 2010;15(23). pii: 19583.

21. Rappold AG, Stone SL, Cascio WE, Neas LM, Kilaru VJ, Carraway MS, et al. Peat bog wildfire smoke exposure in rural North Carolina is associated with cardiopulmonary emergency department visits assessed through syndromic surveillance. Environ Health Perspect. 2011;119(10):1415-20.

22. Buehler JW, Whitney EA, Smith D, Prietula MJ, Stanton SH, Isakov AP. Situational uses of syndromic surveillance. Biosecur Bioterror. 2009;7(2):165-77.

23. Greer SL, Fahy N, Elliott HA, Wismar M, Jarman H, Palm W. Everything you always wanted to know about European Union health policies but were afraid to ask. London: World Health Organization European Observatory on Health Systems and Policies; 2014.

24. Council of the European Union. Council conclusions on common values and principles in European health systems. Off J Eur Union. 2006;C146:1-4.

25. European Parliament and Council of the European Union. Decision on serious crossborder threats to health and repealing Decision No 2119/98/EC (1082/2013/EU). Off J Eur Union. 2013; L 293:1-15.

26. European Commission. Health Programme 2008-2013. Together for Health; 2007 [cited 26 June 2015]. Available from: http://ec.europa.eu/health/ ph_programme/documents/prog_booklet_en.pdf.

27. Brand H. From 'public health in Europe' to 'European public health'. Eur J Public Health. 2010;20(2):127-8.

28. European Commission Consumers, Health, Agriculture and Food Executive Agency. Project database [Internet]; 2012 [cited 26 June 2015]. Available from: http://ec.europa.eu/chafea/projects/database.html.

29. European Union. Generic Public Health Preparedness in Europe. Luxembourg: European Union; 2011 [cited 26 June 2015]. Available from: http://ec.europa.eu/chafea/documents/health/leaflet/generic_preparedness.pdf. 
30. SIDARTHa [Internet]; 2015 [cited 26 June 2015]. Available from: http://www.sidartha.eu.

31. Triple S-AGE [Internet]; 2015 [cited 26 June 2015]. Available from: www.syndromicsurveillance.eu.

32. Walshe K, McKee M, McCarthy M, Groenewegen P, Hansen J, Figueras J, et al. Health systems and policy research in Europe: Horizon 2020. Lancet. 2013;382(9893):668-9.

33. Mackenbach J, McKee M. Introduction. In: Mackenbach J, McKee M, editors. Successes and failures of health policy in Europe. Four decades of divergent trends and converging challenges. Maidenhead: Open University Press; 2013. p. 1-22.

34. Groenewegen PP. Analyzing European health systems: Europe as a research laboratory. Eur J Public Health. 2013;23(2):185-6.

35. Marmor T, Freeman R, Okma K. Comparative perspectives and policy learning in the world of health care. J Comp Pol Anal: Res Practice. 2005;7(4):331-48.

36. Mackenbach J, McKee M, editors. Successes and failures of health policy in Europe Four decades of divergent trends and converging challenges. Maidenhead: Open University Press; 2013.

37. World Health Organization Regional Office for Europe. European Observatory on Health Systems and Policies [Internet]; 2015 [cited 12 July 2015]. Available from: http://www.euro.who.int/en/about-us/partners/observatory.

38. Papanicolas I, Smith PC. Introduction. In: Papanicolas I, Smith PC, editors. Health system performance comparison. An agenda for policy, information and research. Maidenhead: Open University Press; 2013. p. 1-30.

39. Cacace M, Ettelt S, Mays N, Nolte E. Assessing quality in cross-country comparisons of health systems and policies: towards a set of generic quality criteria. Health Policy. 2013;112(1-2):156-62. 



\section{CHAPTER 2}

\section{A new (European) Syndromic Surveillance Definition}

published as:

Triple S Project.* Assessment of syndromic surveillance in Europe. Lancet. $2011 ; 378(9806): 1833-4$.

* Sala Soler M, Fouillet A, Viso AC, Josseran L, Smith GE, Elliot AJ, McMenamin J, Ziemann A, Krafft T 


\section{Assessment of syndromic surveillance in Europe}

Potential public health threats are generally detected through specific surveillance systems based on predetermined diseases and rapid biological confirmation. However, such systems are not rapid and they do not detect all health hazards, especially unexpected threats. Syndromic surveillance can support public health professionals in this endeavour.

The idea was first put into practice in the late 1990s with the aim of providing early warning of disease outbreaks and potential bioterrorist actions (1). Progressively, such surveillance systems have taken a wider public health approach, focused not only on provision of early alerts but also on the reassurance of stakeholders and the public about health effects, ranging from infectious diseases to environmental hazards (2).

Syndromic surveillance systems rely on near real-time automated data collection and analysis from various information sources including general practitioners (3), emergency departments (4), pharmacy sales, telephone help lines (5), web queries (6), and veterinary data (7). These systems monitor the spread and impact, or absence of impact, of known or unknown events in the population on the basis of the presentation of signs and symptoms.

Syndromic surveillance is being increasingly used. Several European countries, including France and the UK $(6,7)$, have already integrated such surveillance into national surveillance programmes on a routine basis. The organisation of public health systems across the European Union (EU) drives diverse approaches to syndromic surveillance. This diversity makes comparison of results difficult but offers the opportunity to learn from the variety of experiences and to identify good practice and factors leading to success.

With the aim of increasing the European capacity for real-time or near real-time surveillance and monitoring of the health burden of expected and unexpected healthrelated events, a European project, co-financed by the Public Health Programme through the Executive Agency for Health and Consumers, was launched in 2010. The Triple-S (Syndromic Surveillance Survey, assessment towards guidelines for Europe, grant agreement GA 2009.11.12) project (8), coordinated by the French Institute for Public Health Surveillance, includes 24 organisations from 13 countries. This project addresses both human and animal health, and offers a unique opportunity to initiate knowledge exchange between experts from both disciplines. The project complements previous EU work: the EuroMOMO project, based on public health mortality monitoring systems (9); and SIDARTHa, focused on the development of a European emergency data-based syndromic surveillance system (10).

The project recognises the vast quantity of potential data sources for syndromic surveillance across Europe, and the different public health surveillance approaches; its objective is not to create a single European system but to review and analyse syndromic surveillance activities across member states.

The project consists of an inventory of syndromic surveillance systems to identify reference people in the member states and survey the characteristics of established, pilot, expired, and planned systems. The project relies on a proactive approach, with the organisation of eight country visits to gain an in-depth understanding of selected systems and to stimulate knowledge transfer. The inventory and the country visits aim to develop a network of expert organisations that can support one another or organisations that are 
planning to start or re-establish syndromic surveillance systems in their own countries. The project will thus contribute to the international discussion of the usefulness of the concept, taking into account not only integral factors of such systems but also contextual determinants, such as treatment-seeking behaviour or political environment.

Before this review was undertaken, the consortium decided that an update of the definition of syndromic surveillance generally used (based on the US Centers for Disease Control and Prevention guidelines) was necessary in view of the evolution of syndromic surveillance during the past decade (1). The development of a common definition by experts in the discipline constitutes the first output of the project (panel).

The Health and Consumer Directorate General of the European Commission, the European Centre for Disease Prevention and Control, WHO Regional Office for Europe, and the International Society for Disease Surveillance are members of the advisory board, to encourage exchange of practices and skills at both the European and the global level.

All relevant findings from the different systems identified by the project will be integrated into a proposal for a European syndromic surveillance strategy that will aim to improve public health surveillance in the member states to reinforce public health action across Europe.

\section{Panel: Definition of syndromic surveillance}

A real-time (or near real-time) collection, analysis, interpretation, and dissemination of health-related data to enable the early identification of the impact (or absence of impact) of potential human or veterinary public health threats that require effective public health action.

Syndromic surveillance is based not on the laboratory-confirmed diagnosis of a disease but on nonspecific health indicators including clinical signs, symptoms as well as proxy measures (e.g., absenteeism, drug sales, animal production collapse) that constitute a provisional diagnosis (or "syndrome").

The data are usually collected for purposes other than surveillance and, where possible, are automatically generated so as not to impose an additional burden on the data providers.

This surveillance tends to be non-specific yet sensitive and rapid, and can augment and complement the information provided by traditional test-based surveillance systems. 


\section{References}

1. Buehler JW, Hopkins RS, Overhage JM, Sosin DM, Tong V. Framework for evaluating public health surveillance systems for early detection of outbreaks: recommendations from the CDC Working Group. MMWR Recomm Rep. 2004;53(RR-5):1-11.

2. Elliot AJ, Singh N, Loveridge $P$, Harcourt S, Smith S, Pnaiser R, et al. Syndromic surveillance to assess the potential public health impact of the Icelandic volcanic ash plume across the United Kingdom, April 2010. Euro Surveill. 2010;15(23). pii: 19583.

3. Flamand C, Larrieu S, Couvy F, Jouves B, Josseran L, Filleul L. Validation of a syndromic surveillance system using a general practitioner house calls network, Bordeaux, France. Euro Surveill. 2008;13(25). pii: 18905.

4. Josseran L, Nicolau J, Caillere N, Astagneau P, Brucker G. Syndromic surveillance based on emergency department activity and crude mortality: two examples. Euro Surveill. 2006;11(12):225-9.

5. Smith GE, Cooper DL, Loveridge P, Chinemana F, Gerard E, Verlander N. A national syndromic surveillance system for England and Wales using calls to a telephone helpline. Euro Surveill. 2006;11(12):220-4.

6. Hulth A, Rydevik $G$, Linde A. Web queries as a source for syndromic surveillance. PLoS One. 2009;4(2):e4378. doi: 10.1371/journal.pone.0004378

7. Eidson M, Schmit K, Hagiwara Y, Anand M, Backenson PB, Gotham I, et al. Dead crow density and West Nile virus monitoring, New York. Emerg Infect Dis. 2005;11(9):1370-5.

8. Triple S-AGE [Internet]; 2011. Available from: www.syndromicsurveillance.eu.

9. Mazick A, Workshop on mortality monitoring in Europe. Monitoring excess mortality for public health action: potential for a future European network. Euro Surveill. $2007 ; 12(1)$. pii $=3107$.

10. Krafft T, Garcia-Castrillo Riesgo L, Fischer M, Kraemer A, Lippert F, Vergeiner G, et al. Emergency data-based syndromic surveillance in Europe. Eur J Public Health. 2008;18(Supplement 1):187-8. 


\section{CHAPTER 3}

\section{A European Syndromic Surveillance System Concept}

published as:

Ziemann A, Rosenkötter N, Garcia-Castrillo Riesgo L, Schrell S, Kauhl B, Vergeiner G, Fischer M, Lippert FK, Krämer A, Brand H, Krafft T. A concept for routine emergency-care data-based syndromic surveillance in Europe. Epidemiol Infect. 2014;142:2433-46. 


\section{Abstract}

We developed a syndromic surveillance (SyS) concept using emergency dispatch, ambulance and emergency-department data from different European countries. Based on an inventory of sub-national emergency data availability in 12 countries, we propose framework definitions for specific syndromes and a SyS system design. We tested the concept by retrospectively applying cumulative sum and spatio-temporal cluster analyses for the detection of local gastrointestinal outbreaks in four countries and comparing the results with notifiable disease reporting. Routine emergency data was available daily and electronically in 11 regions, following a common structure. We identified two gastrointestinal outbreaks in two countries; one was confirmed as a norovirus outbreak. We detected 1/147 notified outbreaks. Emergency-care data-based SyS can supplement local surveillance with near real-time information on gastrointestinal patients, especially in special circumstances, e.g. foreign tourists. It most likely cannot detect the majority of local gastrointestinal outbreaks with few, mild or dispersed cases.

Key words: Gastrointestinal infections, public health, surveillance system. 


\section{Introduction}

Syndromic surveillance (SyS) of pre-diagnostic cases based on signs and symptoms or health-related behaviour is a supplementary approach for timely detection of public health threats and for monitoring events with potential public health impact if information from other surveillance systems are not yet or not at all available (1). SyS can provide a flexible and cost- effective way to gain timely information about the health impact of known and unknown, communicable and non-communicable, natural and man-made health threats $(2,3)$.

The European landscape of public health surveillance mainly consists of three parallel schemes. The first scheme comprises the specific communicable disease surveillance systems of European Union member states (MS) that provide information on confirmed cases following a common case definition to the European Surveillance System (TESSy) (4). The second scheme is different reporting systems through which MS give account of communicable or non-communicable events to inform other MS and European institutions, e.g. the Early Warning and Response System (EWRS) (5). The third scheme comprises unspecific information collated by European networks of different countries, e.g. Influenzanet for self-reported influenza symptoms (6), or EuroMOMO for mortality monitoring (7), and by the Medical Information System (MedISys) that automatically screens online news wires concerning health events (8). SyS is accomplished in MS at the local, regional, and national levels (9). A systematic approach towards European SyS could support timely, comparable, cross-border surveillance.

Routinely collected emergency-care data from (i) emergency medical dispatch (EMD) centres, (ii) ambulance or emergency medical services (EMS), and (iii) emergency departments (ED) can be a valuable source for SyS. Across Europe, emergency-care data is available following a common structure (10). The biggest advantage is the opportunity of real-time reporting of electronic emergency data that can offer timelier and more frequent information compared to established traditional surveillance systems, e.g. based on sentinel doctors (2). It provides data based on a form of clinical assessment, e.g. working diagnoses from emergency physicians (EP), which have a higher specificity for SyS compared to non-clinical data from, e.g. over-the-counter drug sales (11).

We aimed at developing the first concept for SyS based on three routine emergency data sources that is applicable across Europe. We describe the development of the SyS concept and present results of a case study testing the SyS concept using the example of local gastrointestinal outbreak detection.

\section{Methods}

\section{SyS system concept}

Inventory of emergency data availability in Europe

We asked regional (sub-national) emergency service representatives in 12 countries (Austria, Belgium, Czech Republic, Denmark, Finland, France, Germany, Hungary, Italy, Norway, Spain, Turkey) to assess availability and content of routine datasets collected in EMD, EMS, and ED. Using a semi-standardized survey we asked for the method of data collection, i.e. manual or electronic, the frequency of data availability, e.g. daily, and the available data fields in the routine datasets. 


\section{Syndrome definition}

Based on the inventory, we defined syndromes of potential public health relevance that could be generated using routine emergency data. Based on a focus group discussion with emergency-care and public-health experts from across Europe and examples from the literature, we developed recommendations for generating syndromes based on the most common diagnostic coding systems used in EMD, EMS, and ED, i.e. Advanced Medical Priority Dispatch System (AMPDS), versions 11.3 and 12.0 (Priority Dispatch Inc., USA), International Classification of Disease (ICD) 9th and 10th revisions, chief complaints based on Canadian Triage and Acuity Scale (CTAS), and the Minimum Dataset for Emergency Physicians (MIND).

\section{SyS system design}

Based on a review of the literature and material published on existing SyS systems and a consultation with European emergency-care, public-health and information technology experts, we developed a design concept for an emergency data-based SyS system. We defined a minimum standard dataset as input for the SyS system that is applicable for EMD, EMS and ED, defined the data flow, selected statistical analytical methods for detecting unusual aberrations, and described ways of reporting the output.

\section{Case study on local gastrointestinal outbreak detection}

We tested our SyS concept for EMD, EMS and ED, and for different syndromes and purposes, based on retrospective analyses of historical data from regional emergency systems in four countries (12). In this paper we present the results of a case study on local gastro-intestinal outbreak detection.

\section{Datasets}

We analysed data from the EMD centre in the state of Tyrol, Austria (EMD-AT dataset), data from EMS staffed by EP in the state of Tyrol, Austria (EP-AT dataset), the county of Goeppingen, Germany (EP-DE dataset) and the country of Belgium (EPBE dataset), and data from an ED in a university hospital in the city of Santander, Spain (ED-ES dataset). Table 1 describes the main characteristics of the datasets.

\section{Gastrointestinal syndrome case definition}

Table 2 details the definition of gastrointestinal syndrome cases for five common emergency-care coding systems as an example for a syndrome that can be generated based on routine emergency-care data. An emergency case which received any code included in Table 2 was included in the case study. 


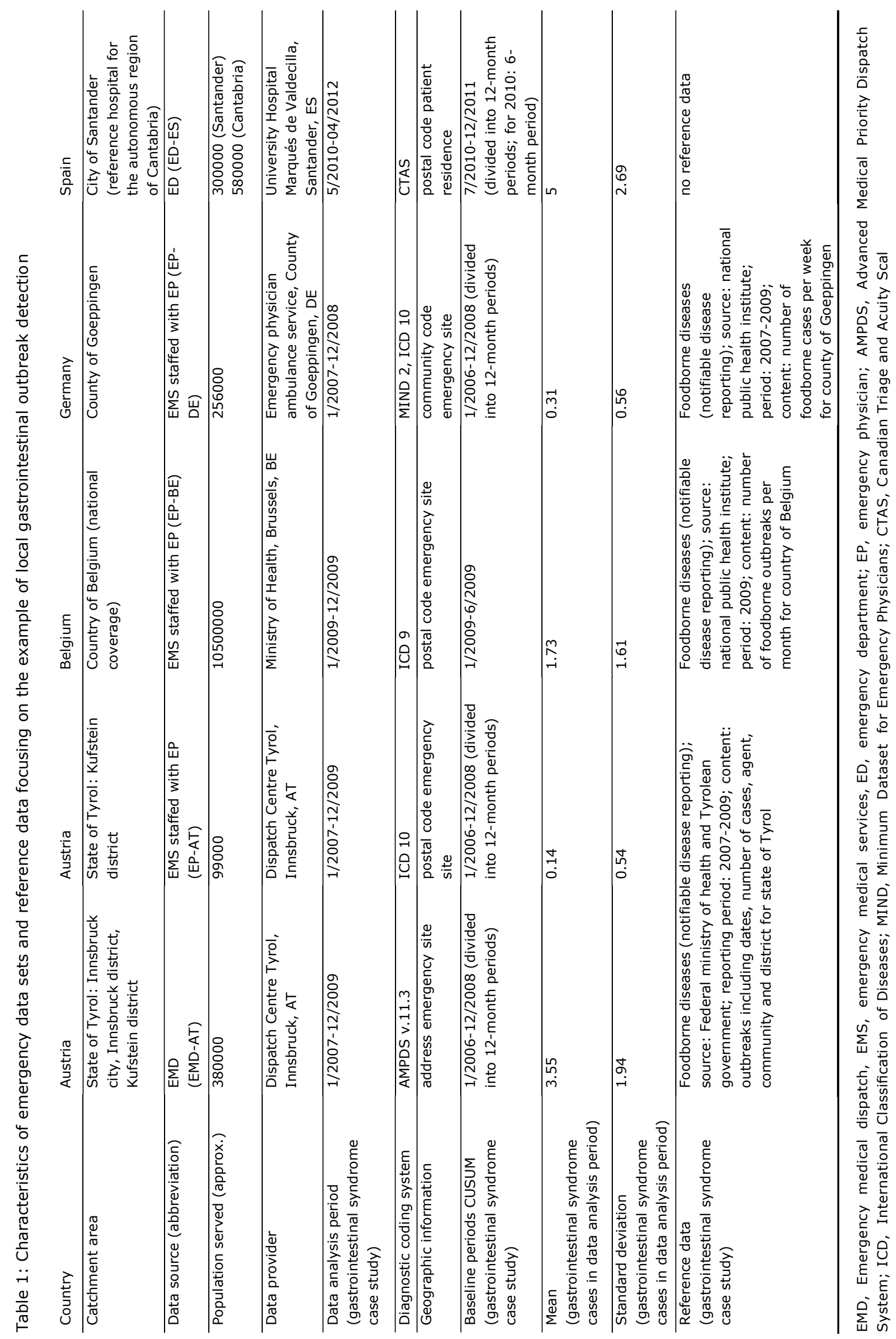


As a first step, three detection algorithms based on cumulative sums were applied for the analysis of aberrations in the time series of gastrointestinal syndrome cases: C1, C2, and C3 based on short-term baselines (13), and two cumulative sum algorithms based on longer baselines, one for normal (CUSUM-N) and one for Poisson-distributed data (CUSUM-P) (14). If the distribution of the datasets for a specific syndrome was neither normal nor Poisson distributed, as was the case for gastrointestinal syndrome cases, we applied all algorithms in parallel. The CUSUM algorithms were enhanced with the fast initial response (FIR) technique which ensures that large chart values do not inflate following values preventing the production of excessive signals (15). In the case study the algorithms were applied retrospectively. We analysed periods of six (EP-BE dataset) or 12 (EMD-AT, ED-ES datasets) months produced a daily CUSUM value. For each analysis period, we calculated baseline means to which the actual values were compared, based on the 6 or 12 months preceding the analysis period (Table 1 ). For the CUSUM-P analysis, the accepted mean was defined close to the actual mean and the threshold value $h$ was defined by look-up procedure in the table of Lucas (16). The temporal aberration detection algorithms have been applied using Microsoft Excel 2003 (Microsoft Corp., USA).

\section{Spatio-temporal cluster detection algorithm}

In a second step, outbreak periods that were identified based on temporal aberration detection analysis (see definition of outbreaks in the next section) were analysed by a prospective spatio-temporal scan statistic (17). The scan statistic process can be explained as a cylindrical scanning window that moves flexibly over the study area. The width of the cylinder base represents the geographical area and the height represents the time period which is scanned. The scan statistic evaluates for all possible cylinder locations and sizes if an observed cluster of cases is caused by chance. The scan statistic can be applied to different levels of spatial aggregation of cases. In the case of spatially aggregated datasets, the cases are concentrated on the centroids of an area. In our case study, a prospective spatio-temporal Bernoulli model-based scan statistic was applied to the exact addresses of the emergency sites in the EMD-AT dataset. A prospective spatio-temporal Poisson model was applied to the EP-AT, EP-BE, EP-DE, and ED-ES datasets, based on the centroids of each administrative area (Table 1) (17). During the scanning process the rates of gastrointestinal cases divided by the total number of emergency cases within the scanning window were compared to the rates outside of the window. The baseline populations were generated using the total number of emergencies in the previous 12 months (EP-AT, EP-DE) and the previous 6 months (EP-BE, ED-ES). The likelihood that a cluster exists by chance was characterized by a $P$ value based on 999 MonteCarlo simulations (17).

For each syndrome, different parameters have to be defined for detecting relevant clusters. For local gastrointestinal outbreak detection, only clusters with the parameters of 1 day temporal length, enclosing a circular area of up to $1 \mathrm{~km}$ radius, and with a significance level of $\mathrm{P}<0.001$ that the cluster exists by chance were defined as relevant. Pre-tests with different parameters showed that for longer and larger cluster sizes the number of cases that formed a cluster was too low and/or the 
cases were scattered over too large an area to reflect a true positive outbreak. The analyses were performed using $\operatorname{SaTScan}^{\mathrm{TM}}$ (v. 9.1.1., M. Kulldorff and Information Management Services Inc., USA). The identified spatio-temporal clusters were visualized using ESRI ArcGIS ${ }^{\circledR}$ v. 10.1. (Environmental Systems Research Institute Inc., USA).

Table 2: Gastrointestinal syndrome definition for five common emergency care coding systems (21)

\begin{tabular}{|c|c|}
\hline Coding System & Codes included for gastrointestinal syndrome (Boolean operator: OR) \\
\hline AMPDS $11.3,12.0$ & A1 Abdominal Pain \\
\hline ICD 9 & $\begin{array}{l}\text { 001 Cholera } \\
002 \text { Typhoid and paratyphoid fevers } \\
003 \text { Other salmonella infections } \\
004 \text { Shigellosis } \\
008.5 \text { Bacterial enteritis unspecified } \\
005 \text { Other food poisoning (bacterial) } \\
\text { 005.9 Food poisoning unspecified } \\
\text { 008.6 Enteritis due to specified virus } \\
\text { 008.69 Enteritis due to other viral enteritis } \\
\text { 009.2 Infectious diarrhoea } \\
\text { 009.3 Diarrhoea of presumed infectious origin } \\
\text { 787.0 Nausea and vomiting } \\
\text { 787.01 Nausea with vomiting } \\
\text { 787.02 Nausea alone } \\
\text { 787.03 Vomiting alone } \\
\text { 787.81 Diarrhoea } \\
\text { 538.9 Other and unspecified non-infectious gastroenteritis and colitis }\end{array}$ \\
\hline ICD 10 & $\begin{array}{l}\text { A00 Cholera } \\
\text { A01 Typhoid and paratyphoid fevers } \\
\text { A02 other salmonella infections } \\
\text { A03 Shigellosis } \\
\text { A04 other bacterial intestinal infections } \\
\text { A05 Other bacterial foodborne intoxications, not elsewhere classified } \\
\text { A08 Viral and other specified intestinal infections } \\
\text { A09 Diarrhoea and gastroenteritis of presumed infectious origin } \\
\text { R11 Nausea and vomiting } \\
\text { K52 Other non-infective gastroenteritis and colitis } \\
\text { K52.9 Non-infective gastroenteritis and colitis, unspecified } \\
\text { T62.9 Noxious substance eaten as food, unspecified }\end{array}$ \\
\hline MIND II & $\begin{array}{l}\text { 4.1 Abdominal disorders, acute abdomen } \\
\text { 4.2 Abdominal disorders, gastrointestinal bleeding } \\
\text { 4.3 Abdominal disorders, colic } \\
\text { 4.4 Abdominal disorders, other disease abdomen } \\
\text { 6.2 Metabolic disease, dehydrated }\end{array}$ \\
\hline CTAS & $\begin{array}{l}\text { Abdominal pain adults } \\
\text { Abdominal pain children } \\
\text { Diarrhoea } \\
\text { Vomiting }\end{array}$ \\
\hline
\end{tabular}

AMPDS, Advanced Medical Priority Dispatch System; ICD, International Classification of Diseases; MIND, Minimum Dataset for Emergency Physicians; CTAS, Canadian Triage and Acuity Scale. 
We developed a decision tree as suggested by Meyer et al. (18) and Ansaldi et al. (19) to define inclusion criteria for outbreaks based on the signals given by, first, the temporal and, second, the spatio-temporal detection algorithm. For the case of local gastrointestinal outbreak detection, these were (i) at least 2 days of consecutive temporal aberration detection signals, or (ii) days with an exceptionally high aberration in case numbers from the mean ( $>3$ standard deviations (S.D.) from the baseline mean of the previous 6 or 12 months), and (iii) outbreaks identified by the temporal aberration analyses with corresponding spatio-temporal clusters.

\section{Validation of outbreaks}

The comparison with reference data from other (traditional) surveillance systems can give additional assurance that a signal could represent a real event. For the case study on local gastrointestinal outbreaks, we compared the detected outbreaks with notifiable surveillance reports of foodborne diseases. This reference data was available for Tyrol (Austria), Belgium, and Goeppingen (Germany) (20) (Table 1).

\section{Results}

\section{SyS system concept}

Availability of emergency data

Routine electronic data was available daily in 11 of 12 regions from EMD, EMS and/or ED (Table 3). Information on the patients' chief complaints was available daily and electronically in ten systems, information on age and sex in nine systems (Table 3 ). Although the datasets comprised common data fields across Europe such as date, age, sex, and diagnostic information, the items were defined differently. In particular, diagnostic information varied. Sometimes international coding systems were used, and sometimes data was collected following regional or national coding systems (Table 1).

\section{SyS system design}

We defined a standard dataset for SyS that can be generated based on routine data collected in the majority of EMD, EMS, or ED across Europe: (1) date, (2) syndrome, (3) geographical reference, (4) modifier I: age, (5) modifier II: sex, (6) modifier III: severity.

Figure 1 shows the generic functions and data flow of the automated SyS system. The system can be implemented by emergency institutions using the institution's already established health information technology infrastructure. The emergency institution is supposed to programme a permanent, daily translation between the emergency database and the surveillance system following the standard SyS dataset, e.g. an extract transform load (ETL) process. Afterwards, the syndromic data should automatically be analysed by applying temporal and spatio-temporal aberration detection algorithms in parallel. The proposed algorithms can be operationalized 
using open source software such as R (21) and SaTScan, or can be programmed directly in other, already applied data analysis software. The parameters of the algorithms have to be calculated once for each monitored syndrome and each emergency dataset, based on historical emergency data. During regular operation of the SyS system, these parameters should be updated regularly and after changes in the data collection procedure. The outputs of the SyS analyses are statistical signals that can be displayed in tables, charts and maps, which can be disseminated within the emergency-care institution and to the local/regional public health authority. Reporting can be accomplished by establishing a regular automatic email message, by incorporating the results in already established reports, or by allowing stakeholders to access a virtual dashboard online that is automatically updated on a regular basis. The public health authority and/or emergency institution decide if the signals could represent a real event following a pre-defined decision tree for each syndrome. The public health authority can incorporate SyS alerts into existing surveillance systems and response procedures. The emergency institution can use the information for resource planning. At the time of writing this paper, two institutions have implemented an automatic SyS system following this concept, the EMD centre of the State of Tyrol, Austria, and the ED of the University Hospital in Santander, Spain.

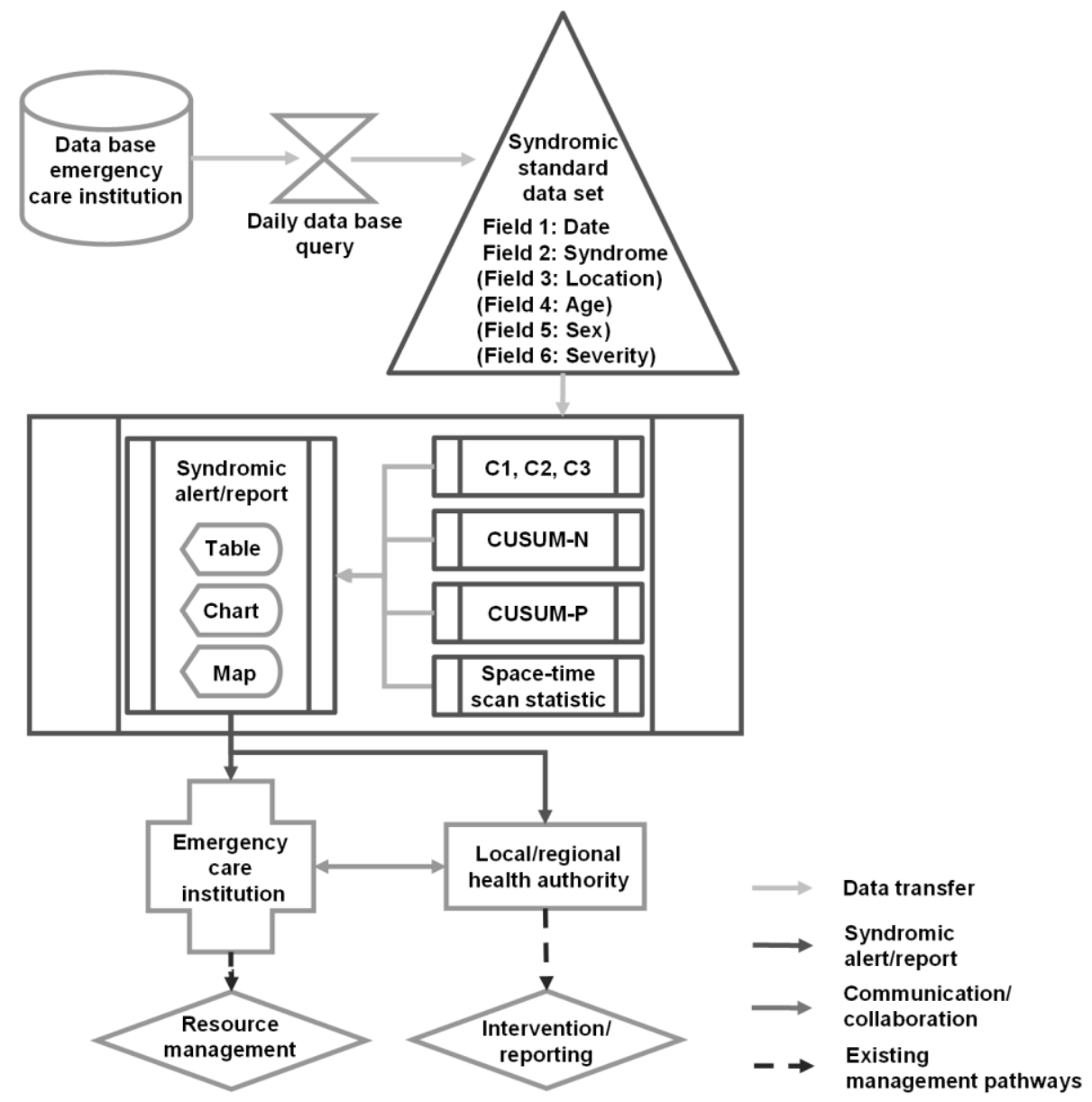

Figure 1: Concept of an automated emergency data-based SyS system. 


\section{Case study on local gastrointestinal outbreak detection}

The case study showed that the case numbers in the datasets based on data from EMS staffed with EP in the Austrian (EP-AT dataset) and the German (EP-DE dataset) regions, with an average of 0.14 and 0.31 cases per day, respectively, were too low for providing valid results based on the temporal aberration detection analysis. Figure 2 shows the time series of the number of gastrointestinal syndrome cases and the signals of the temporal aberration detection analyses for the EMD-AT, EP-BE and EDES datasets.

Table 3: Availability of selected electronic emergency care information from three sources (EMD, EMS, ED) in regional emergency institutions in 12 countries (status: June 2009)

\begin{tabular}{|c|c|c|c|c|c|c|}
\hline \multirow[b]{2}{*}{ Country } & \multirow[b]{2}{*}{ Region } & \multirow[b]{2}{*}{$\begin{array}{l}\text { Data } \\
\text { source }\end{array}$} & \multicolumn{4}{|c|}{ Data availability } \\
\hline & & & date & $\begin{array}{l}\text { chief } \\
\text { complaint/ } \\
\text { working } \\
\text { diagnosis }\end{array}$ & age & sex \\
\hline \multirow[t]{3}{*}{ Austria } & $\begin{array}{l}\text { State of Tyrol (City of Innsbruck, } \\
\text { District of Innsbruck, District of } \\
\text { Kufstein) }\end{array}$ & EMD & daily & daily & daily & daily \\
\hline & District of Kufstein & EMS (EP) & daily & daily & daily & daily \\
\hline & District of Kufstein & $\mathrm{ED}$ & daily & n.a. & daily & daily \\
\hline \multirow[t]{3}{*}{ Belgium } & Province of Flemish-Brabant & EMD & n.a. & n.a. & n.a. & n.a. \\
\hline & City of Leuven & EMS (EP) & daily & weekly & daily & daily \\
\hline & City of Leuven & ED & daily & daily & daily & daily \\
\hline Czech & City of Prague & EMD & daily & daily & weekly & weekly \\
\hline \multirow[t]{2}{*}{ Republic } & City of Prague & EMS & daily & weekly & - & - \\
\hline & City of Prague & ED & n.a. & n.a. & n.a. & n.a. \\
\hline \multirow[t]{3}{*}{ Denmark } & Capital Region of Denmark & EMD & daily & daily & - & - \\
\hline & Capital Region of Denmark & EMS (EP) & daily & daily & daily & daily \\
\hline & Capital Region of Denmark & ED & daily & - & daily & daily \\
\hline \multirow[t]{3}{*}{ Finland } & City of Kuopio & EMD & daily & daily & - & daily \\
\hline & City of Kuopio & EMS (EP) & daily & - & weekly & weekly \\
\hline & City of Kuopio & ED & daily & daily & daily & daily \\
\hline \multirow[t]{3}{*}{ France } & District of Hauts-de-Seine & EMD & n.a. & n.a. & n.a. & n.a. \\
\hline & District of Hauts-de-Seine & EMS (EP) & weekly & - & weekly & weekly \\
\hline & n.a. & ED & n.a. & n.a. & n.a. & n.a. \\
\hline \multirow[t]{3}{*}{ Germany } & County of Goeppingen & EMD & daily & - & - & - \\
\hline & County of Goeppingen & EMS (EP) & daily & daily & daily & daily \\
\hline & County of Goeppingen & ED & daily & - & daily & daily \\
\hline \multirow[t]{3}{*}{ Hungary } & national level & EMD & n.a. & n.a. & n.a. & n.a. \\
\hline & national level & EMS (EP) & monthly & - & monthly & $\begin{array}{l}\text { month } \\
\text { ly }\end{array}$ \\
\hline & City of Budapest & ED & daily & - & daily & daily \\
\hline \multirow[t]{3}{*}{ Italy } & Province of Genoa & EMD & daily & daily & - & - \\
\hline & Province of Genoa & EMS (EP) & monthly & - & monthly & $\begin{array}{l}\text { month } \\
\text { ly }\end{array}$ \\
\hline & Province of Genoa & ED & n.a. & n.a. & n.a. & n.a. \\
\hline \multirow[t]{3}{*}{ Norway } & City of Bergen & EMD & daily & daily & daily & daily \\
\hline & City of Bergen & EMS & daily & - & - & - \\
\hline & City of Bergen & ED & n.a. & n.a. & n.a. & n.a. \\
\hline \multirow[t]{3}{*}{ Spain } & Autonomous Region of Cantabria & EMD & daily & daily & daily & daily \\
\hline & Autonomous Region of Cantabria & EMS (EP) & weekly & weekly & weekly & weekly \\
\hline & Autonomous Region of Cantabria & ED & daily & daily & daily & daily \\
\hline \multirow[t]{3}{*}{ Turkey } & City of Antalya & EMD & monthly & n.a. & n.a. & n.a. \\
\hline & n.a. & EMS & n.a. & n.a. & n.a. & n.a. \\
\hline & n.a. & ED & n.a. & n.a. & n.a. & n.a. \\
\hline
\end{tabular}

EMD, Emergency medical dispatch, EMS, emergency medical services, ED, emergency department; EP, emergency physician; n.a., information not available; -, data not available. 

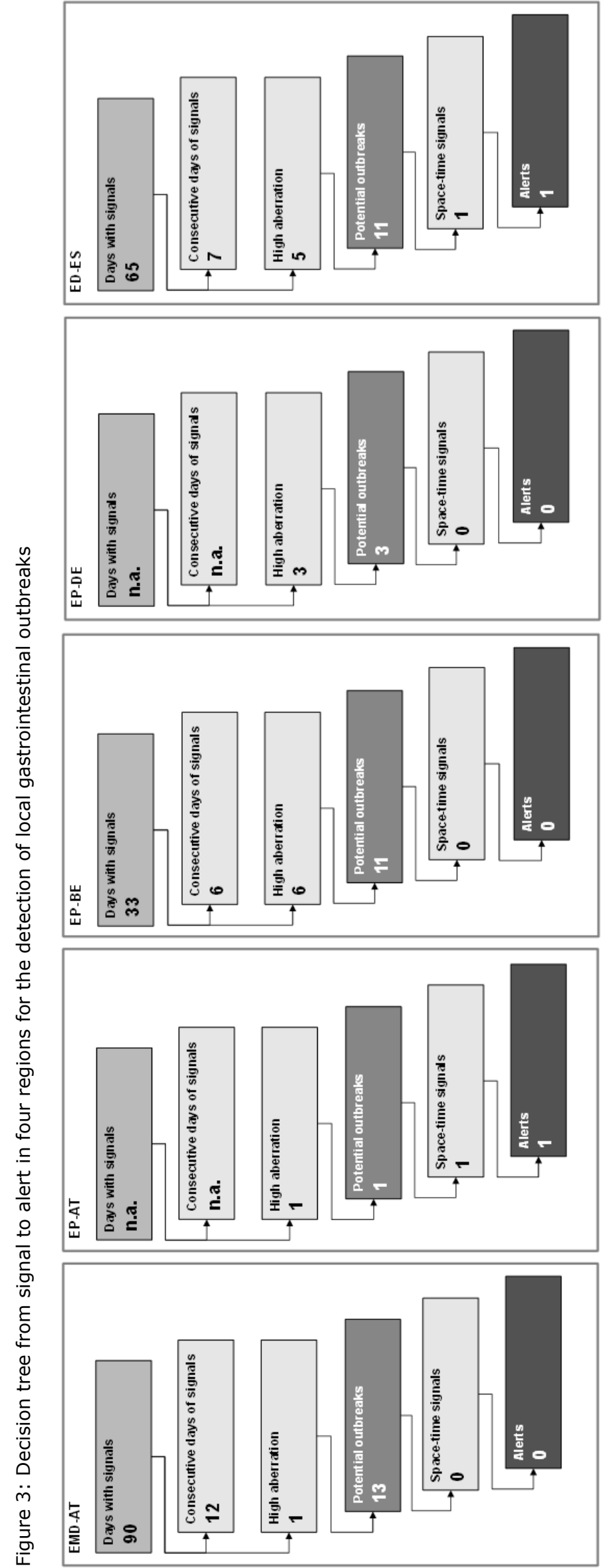
The temporal aberration detection analyses resulted in many signals. When applying the decision tree to identify outbreaks, there were many events with high aberration from the mean and with signals on at least 2 consecutive days. When applying the spatio-temporal analysis during these outbreak periods, we were able to further narrow down the number of relevant outbreaks. Figure 3 provides an overview on the number of signals and the application of the decision tree for each dataset. One outbreak was located in Tyrol, Austria (EP-AT) (14 February 2007, 12 cases within a circle of $0 \mathrm{~km}$ radius, $\mathrm{P}<0.0001$ ), and one in Santander, Spain (3 August 2010, seven cases within a circle of $0.68 \mathrm{~km}$ radius or distribution across postal code areas of $2.2 \mathrm{~km}^{2}, \mathrm{P}<0.0001$ ). Figure 4 exemplifies the cluster in Santander, Spain.

The comparison with notifiable disease reporting data confirmed the alert on 14 February 2007 as a norovirus outbreak in a group of foreign students who stayed in one hotel in the city of Kufstein ( $n=26$ cases). The alert was not confirmed by the EMD-AT dataset which refers to the same region. Two subsequent norovirus outbreaks in the following days in two foreign tourist groups in the same hotel ( $\mathrm{n}=$ 10 and $n=53$ cases) were not identified in the syndromic datasets. No other notified foodborne outbreak in Tyrol, Austria $(n=42)$, and Belgium ( $n=105$ ) could ultimately be linked to signals in the syndromic data-sets. The reference data from Goeppingen, Germany did not provide the number of outbreaks.

\section{Discussion}

\section{SyS system concept}

We developed the first concept for a SyS system based on routinely collected emergency medical care data from EMD, EMS and ED for different countries in Europe.

Routine emergency data was available in many regions in Europe in electronic form and on a daily basis. It provided relevant information for SyS, such as date and geographical information and the patients' chief complaints. We defined recommendations for syndrome coding, based on the most common coding systems in emergency care, and designed a concept for an emergency data-based SyS system able to be implemented at the local/regional level in Europe. Two regional emergency institutions in Austria and Spain have initially implemented an automatic SyS system following our concept.

As the emergency data inventory revealed differences in data coding and availability across Europe, we conceptualized the system to be implemented at single emergency institutions or in one jurisdiction. This allows for raw data to be analysed in the emergency institution, respecting data privacy. This flexibility of the concept supports a relatively rapid set-up of a SyS system as no agreements or technical connections outside of the emergency institution have to be established. The syndrome definitions based on the most common emergency-care coding systems ease the implementation and support the portability of the SyS concept across Europe. Next to the gastrointestinal syndrome, the expert consortium defined syndromes for respiratory and influenza-like illness, for heat-related illness and unspecific syndrome (= volume of medical cases without specification) $(12,22)$. The results of case studies analysing these syndromes are discussed elsewhere $(23,24)$. 
A

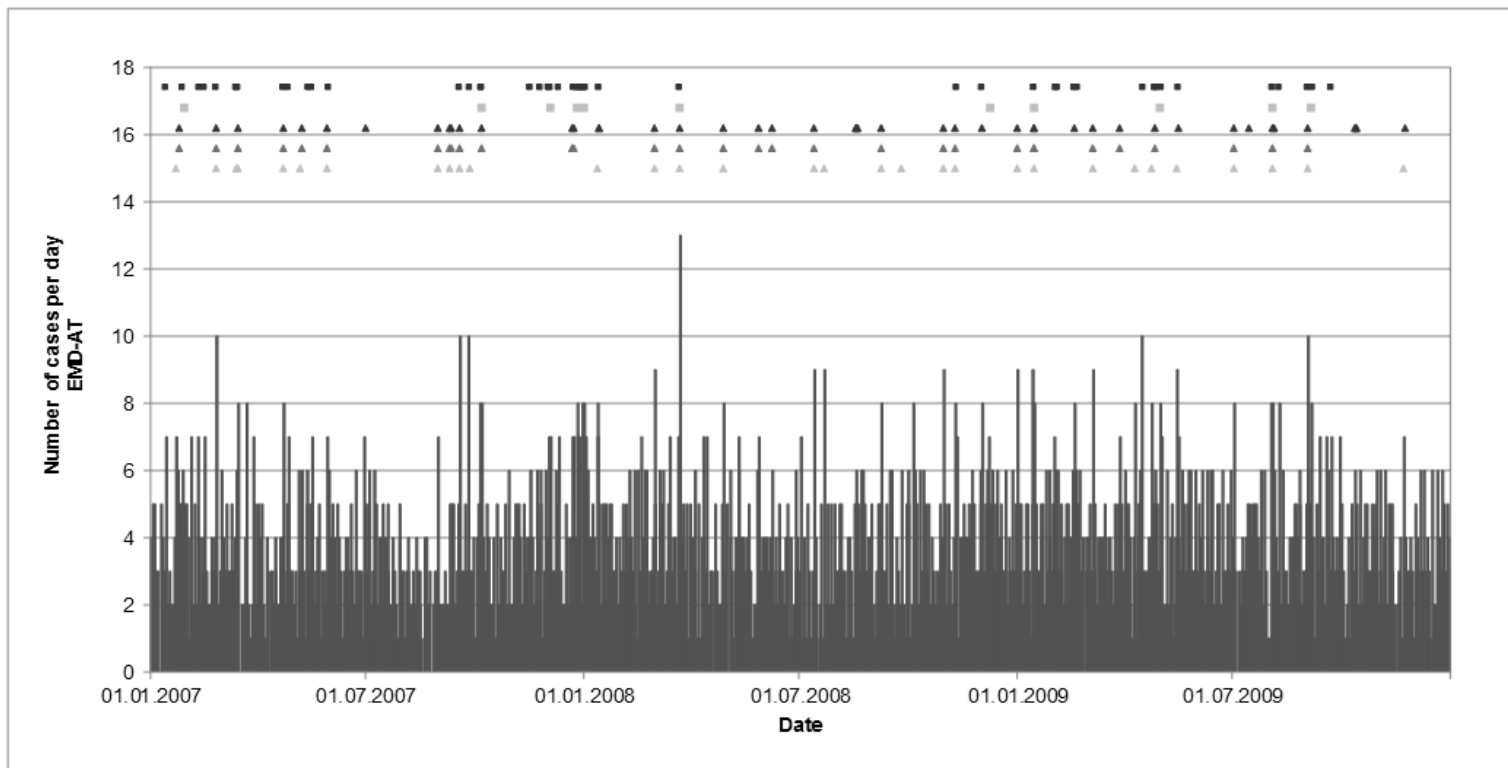

B

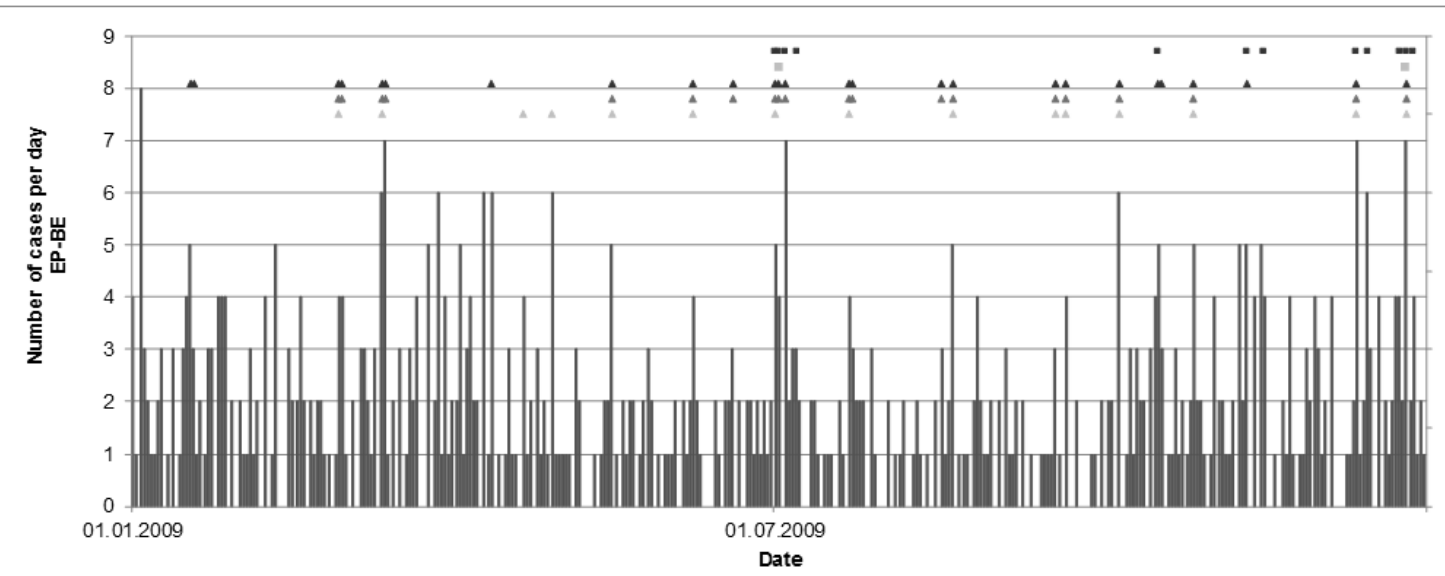

c

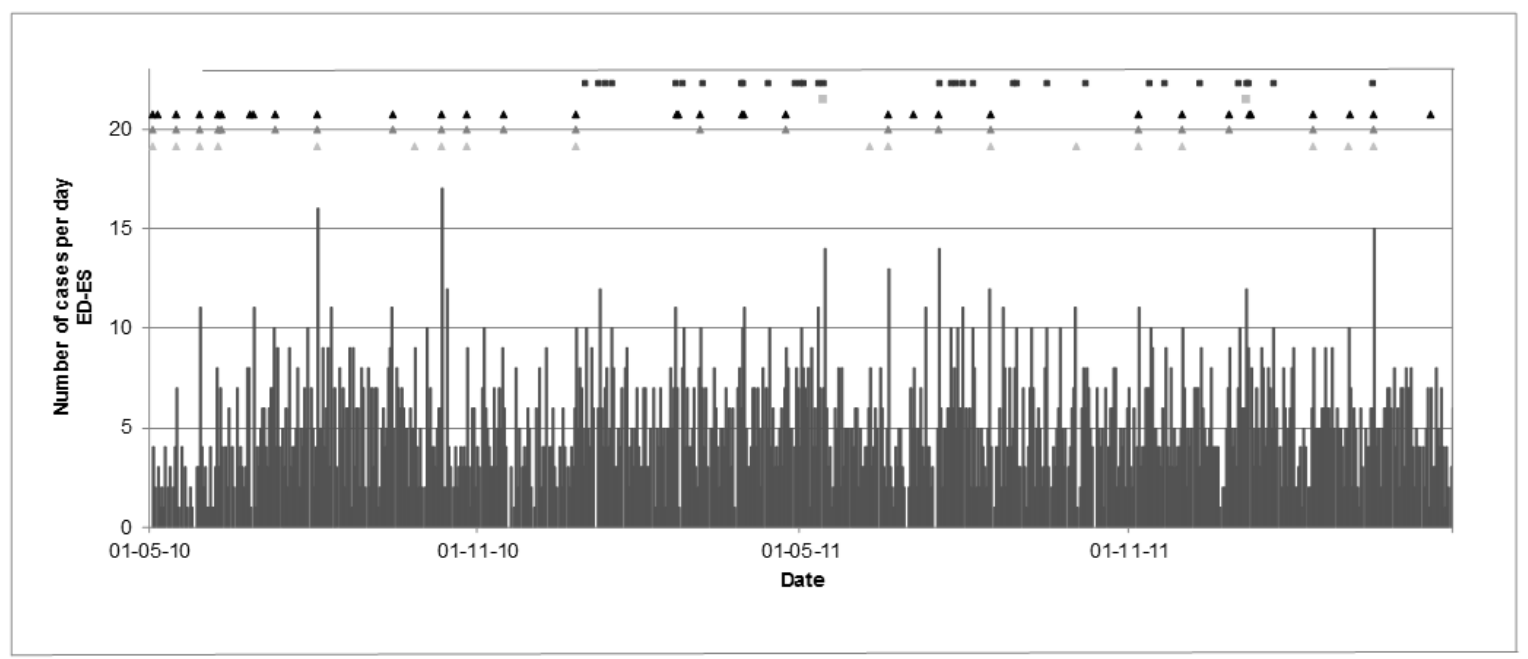

I Gastrointestinal syndrome $\star \mathrm{C} 1$ signal $\triangle \mathrm{C} 2$ signal $\triangle \mathrm{C} 3$ signal $\quad$ CUSUM-N signal $\quad \cdot$ CUSUM-P signal

Figure 2 Time series and temporal aberration detection algorithm signals for local gastrointestinal cases in three regions: (a) state of Tyrol, Austria, based on emergency medical dispatch data (EMD-AT); (b) Belgium (national coverage) based on data from emergency medical services (EMS) staffed with emergency physicians (EP-BE); (c) city of Santander, Spain, based on emergency department data (ED-ES) 

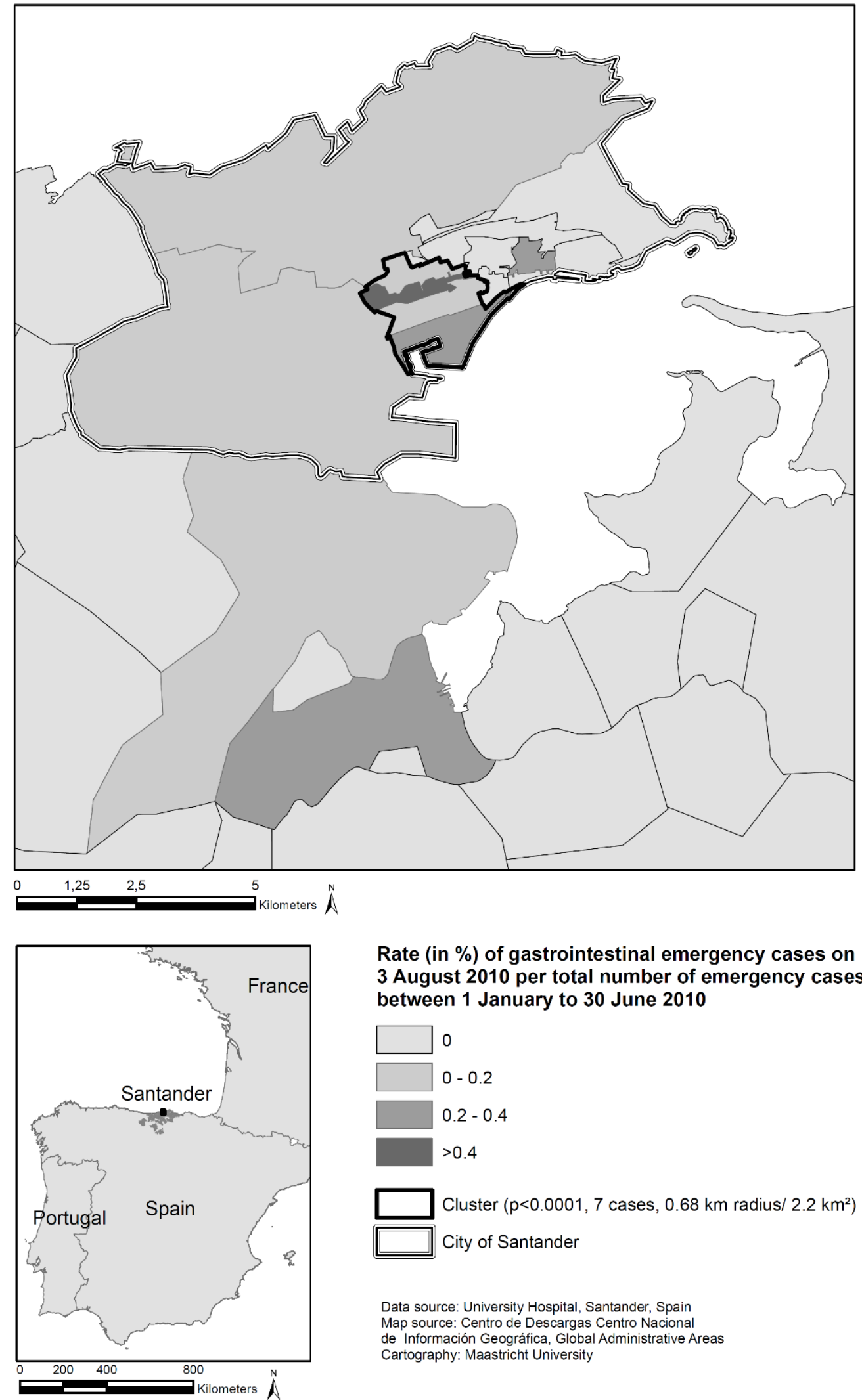

Rate (in \%) of gastrointestinal emergency cases on 3 August 2010 per total number of emergency cases between 1 January to 30 June 2010

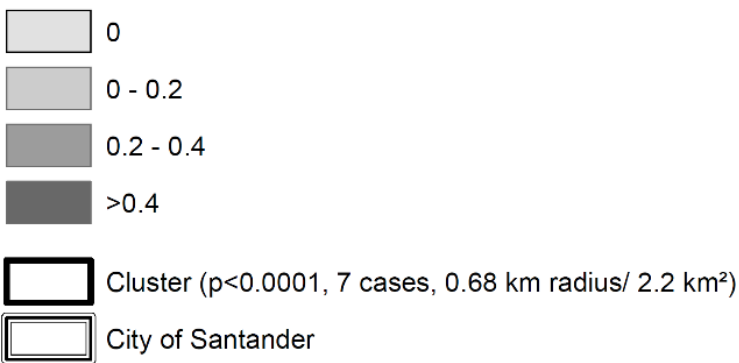

Data source: University Hospital, Santander, Spain

Map source: Centro de Descargas Centro Nacional

de Información Geográfica, Global Administrative Areas

Cartography: Maastricht University

Figure 4: Exemplary spatio-temporal cluster of gastrointestinal syndrome cases in Santander, Spain, on 3 August 2010 and rate of gastrointestinal syndrome cases on 3 August 2010 per total number of ED cases between 1 January and 30 June 2010 per postal code. 


\section{Case study on local gastrointestinal outbreak detection}

Our SyS concept was tested for the detection of local outbreaks of gastrointestinal illness in four regions in Europe. In this case study, we identified two potentially relevant outbreaks. The outbreak identified in Spain could not be confirmed due to missing reference data. The alert in Austria was confirmed as a norovirus outbreak in a group of foreign students. No other notified outbreak was identified by the SyS analyses. This low validity shows that our SyS concept cannot replace traditional surveillance of gastrointestinal diseases.

Gastrointestinal diseases are often the focus of SyS applications (25), pursuing three major purposes: (i) early information on the onset of expected seasonal outbreaks such as winter vomiting disease (26), (ii) situational awareness during potentially health-threatening events such as disasters or mass gatherings (18), and (iii) detection of local gastrointestinal illness clusters (27). Earlier studies suggested that comparatively large outbreaks at the local or regional levels were successfully detected by SyS systems (28). Rather small outbreaks, however, appear to be difficult to detect as Xing et al. (29), Balter et al. (30) and Heffernan et al. (31) found based on ED data. Moreover, in our study most notified outbreaks in the study regions, which mainly consisted of few cases, were not detected by our SyS analyses. Emergency-care data, similar to other health services based data sources for surveillance, are unlikely to reflect outbreaks with few or dispersed cases such as foodborne outbreaks comprised of visitors to a restaurant who later develop symptoms when they are in different areas (30).

Another explanation for the low validity is the fact that emergency-care data sources are not anticipated to catch all gastrointestinal outbreaks as most gastrointestinal illness patients with mild symptoms would self-treat their symptoms or utilize primarycare services. This assumption would suggest additional analysis of other data sources for SyS with a bigger coverage of mild gastrointestinal illness cases. Andersson and colleagues (32) compared three syndromic data sources able to cover people affected by gastrointestinal illness who were not seeking care in Sweden: telephone helplines, web queries and over-the-counter drug sales. This study also confirmed the finding that only larger outbreaks were detected by SyS. From nine point-source outbreaks only the four largest were detected with case numbers between 369 and 27000 . Five smaller outbreaks with case numbers between 100 and 185 were not detected. We could not test our concept on large outbreaks as no outbreaks with more than 53 cases occurred during the study period. The reference data in Belgium and Germany did not provide the number of cases per outbreak.

Emergency care especially comes into contact with gastrointestinal illness in case of severe illness, e.g. during the Shiga toxin-producing Escherichia coli outbreak in Germany in 2011 during which ED reported on bloody diarrhoea cases (33). Further, emergency services are approached by gastrointestinal patients during crisis situations such as the 2003 blackout in the USA (34). In ED in the USA, seasonal increases of gastrointestinal cases are seen during winter suggesting that gastrointestinal patients visit emergency services not only for severe illness but most likely because other health facilities are not accessible, e.g. during Christmas holidays (30). In addition, emergency services cover patients with special characteristics, e.g. as in our case of Austria foreign tourists that might have decided to use emergency care as the easiest point of access to care. Hence, compared to other SyS data 
sources, emergency-care data-based SyS can have an added value for gastrointestinal surveillance if patients with severe symptoms or in special circumstances are using emergency care instead of other health services.

In the case study, we received many temporal signals for aberrations consisting of small case numbers which could not be confirmed by data from notifiable disease surveillance, which was also the case in other studies $(35,36)$. This could be due to the choice or calibration of the statistical methods applied for temporal aberration detection analysis (37). The application of other detection algorithms such as regression analysis or moving averages could yield more valid results. However, we saw the greatest potential to increase validity by additionally applying spatio-temporal detection algorithms which are expected to add information to solely temporal analyses of local gastrointestinal outbreaks as many cases tend to cluster in relatively small areas (38).

Other studies applying spatio-temporal scan statistics detected rather large or severe outbreaks $(36,39)$. In order to enhance the validity of detecting small clusters, adjustment of the analysis parameters was suggested (38). Our case study showed promising results for identifying smaller outbreaks and reducing the number of potential false alerts when applying relatively restrictive parameters to the analysis. This limited our analysis to only detect point-source outbreaks although it increased the probability of receiving alerts for true positive outbreaks. We also tested less restrictive parameters to scan for clusters up to 1 week and up to $5 \mathrm{~km}$ radius but found only insignificant results.

The aggregation of cases to a larger geographical area yields the problem of lower validity of the identified clusters (40). In our case study, the Spanish study area contained both urban and rural areas with very large zip-code areas. If a cluster had been detected comprised of such a large postal code, the risk of it being a false alert is much higher compared to a cluster comprised of only small urban postal code areas. Another limitation in the applied scan statistic is the fixed circular form of the scanning window which cannot identify clusters of another shape. Flexible shapes have been tested but are not commonly used (17). Due to high computing time we applied the prospective spatio-temporal analysis to shorter, previously defined outbreak periods based on the temporal analysis for the whole study area, which might have led to missing outbreaks that cluster in space and time, but are not visible in the purely temporal analysis. This problem would be diminished if the analyses ran automatically.

We are the first to have used run-sheet data from EMS staffed with EP for SyS. Although in two areas the case numbers were too low to perform a valid temporal aberration detection analysis, the data source appears to be promising for SyS. The true positive norovirus outbreak in Tyrol, Austria, was only captured by the data from the EP run sheets and not by the EMD data covering the same area. This indicates a higher specificity of EP-staffed EMS compared to EMD data. It also indicates that SyS based on data sources with such low case numbers tend to detect point-source outbreaks with a high number of cases rather than continuous or propagated source outbreaks with low case numbers or cases dispersed over space and time. We encourage further research using ambulance data for SyS to confirm our findings. 
The case study was performed retrospectively and was not based on results from active automated SyS systems. The performance of the two currently implemented automated systems needs to be evaluated prospectively in the future to further confirm the usefulness of our concept.

\section{Conclusions}

We have provided a practical concept for implementing SyS in Europe based on routine emergency-care data from EMD, EMS and ED that can be used as supplementary and timely surveillance information source at the local/regional level. Emergency-care data-based SyS can supplement local surveillance with near realtime information on gastrointestinal patients, especially in special circumstances or with special treatment-seeking behaviour, e.g. foreign tourists. It should be able to detect large outbreaks and outbreaks comprised of patients with severe symptoms. It is not very likely to detect the majority of local gastrointestinal outbreaks with few, mild or dispersed cases. We recommend using a combination of temporal and spatial outbreak detection algorithms in parallel and to apply a decision tree for initiating public health action based on statistical signals, in order to increase the validity of Sys.

\section{Acknowledgements}

This paper describes results from the SIDARTHa project, which is an initiative of the European Emergency Data (EED) Research Network. We thank all SIDARTHa Consortium partners for their valuable contributions for conceptualizing and testing the SIDARTHa concept. Furthermore, we thank the Belgian Scientific Institute of Public Health and the Tyrolean Government for providing reference data for the case study of gastrointestinal outbreak detection and the Belgian Ministry of Health for providing national ambulance service data for Belgium. We also thank the anonymous reviewer for providing valuable comments to earlier versions of this paper.

This paper arose from the SIDARTHa project which has received funding from the European Union, within the framework of the Public Health Programme (grant agreement no. 2007208). 


\section{References}

1. Triple S Project. Assessment of syndromic surveillance in Europe. Lancet. 2011;378(9806):1833-4.

2. Buehler JW, Whitney EA, Smith D, Prietula MJ, Stanton SH, Isakov AP. Situational uses of syndromic surveillance. Biosecur Bioterror. 2009;7(2):165-77.

3. Ziemann A, Krafft T, Rosenkötter N, Garcia-Castrillo Riesgo L, Vergeiner G, Fischer $M$, et al. Syndromic surveillance. Enhancing public health responsiveness to global change - a European perspective. IHDP Update. 2011:12-8.

4. European Centre for Disease Prevention and Control. The European Surveillance System (TESSy) [Internet]; 2013 [cited 18 June 2013]. Available from: http://ecdc. europa.eu/en/activities/surveillance/tessy/pages/tessy.aspx).

5. European Communities. The Commission Health Emergency Operations Facility: for a coordinated management of public health emergency at EU level. Luxembourg: European Communities; 2007.

6. Influenzanet [Internet]; 2013 [cited 16 November 2013]. Available from: http://influenzanet.eu.

7. EuroMOMO [Internet]; 2013 [cited 16 November 2013]. Available from: http://euromomo.eu.

8. European Commission Joint Research Centre. Medical Information System [Internet]; 2013 [cited 18 June 2013]. Available from: http://medusa.jrc.it/medisys.

9. Fouillet A, Sala-Soler M, Conti S, Kanieff M, Rago G, Perrin J, et al. Inventory of syndromic surveillance systems in Europe by the Triple-S project. Emerg Health Threats J; 2011:10946.

10. Krafft T, Garcia Castrillo-Riesgo L, Edwards S, Fischer M, Overton J, RobertsonSteel I, et al. European Emergency Data Project (EED Project): EMS data-based health surveillance system. Eur J Public Health. 2003;13(3 Suppl):85-90

11. Das D, Metzger K, Heffernan R, Balter S, Weiss D, Mostashari F. Monitoring overthe-counter medication sales for early detection of disease outbreaks--New York City. MMWR Morb Mortal Wkly Rep. 2005;54 Suppl:41-6.

12. Rosenkötter N, Kauhl B, Garcia-Castrillo Riesgo L, Llorca Diaz FJ, Kraan J, Ziemann A, et al., for the SIDARTHa project group. Retrospective data analysis and simulation study as basis for an automated syndromic surveillance system. Results from the SIDARTHa project. Bad Honnef: SIDARTHa Scientific/Technical Coordination Office; 2010 [cited 12 March 2013]. Available from http://www.sidartha.eu/docs/2007208_SIDARTHa_D6_WP61\%20Report\%20data\%20analysis\%20incl\%20WP7\%20case\%20studies.pdf

13. Hutwagner L, Thompson W, Seeman GM, Treadwell T. The bioterrorism preparedness and response Early Aberration Reporting System (EARS). J Urban Health. 2003;80(2 Suppl 1):i89-96.

14. Burkom H. Alerting Algorithms for Biosurveillance. In: Lombardo JS, Buckeridge DL, editors. Disease Surveillance: A Public Health Informatics Approach. Hoboeken New Jersey: John Wiley \& Sons Inc.; 2007. p.159-63. 
15. Lucas JM, Crosier RB. Fast initial response for CUSUM quality-control schemes: Give your CUSUM a head start. Technometrics 2000; 42: 102-7.

16. Lucas JM. Counted Data CUSUM's. Technometrics 1985;27(2):129-44.

17. Kulldorff M. Scan Statistics for Geographical Disease Surveillance: An Overview. In: Lawson AB, Kleinman $K$, editors. Spatial and Syndromic Surveillance. Chichester: Wiley; 2005. p.115-31.

18. Meyer N, McMenamin J, Robertson C, Donaghy M, Allardice G, Cooper D. A multidata source surveillance system to detect a bioterrorism attack during the G8 Summit in Scotland. Epidemiol Infect. 2008;136(7):876-85.

19. Ansaldi F, Orsi A, Altomonte F, Bertone G, Parodi V, Carloni R, et al. Emergency department syndromic surveillance system for early detection of 5 syndromes: a pilot project in a reference teaching hospital in Genoa, Italy. J Prev Med Hyg. 2008;49(4):131-5.

20. Robert Koch Institut. SurvStat [Internet]; 2013 [cited 1 March 2013]. Available from: http://www3.rki.de/SurvStat/.

21. R Development Core Team. R: A language and environment for statistical computing. Vienna: R Foundation for Statistical Computing; 2008.

22. Garcia-Castrillo Riesgo L, Rosenkötter N, Ziemann A, Krafft T, Fischer M, Lippert $F$, et al. The SIDARTHa coding manual. How to generate syndromes based on routinely collected emergency care data for the European syndromic surveillance system SIDARTHa. Bad Honnef: SIDARTHa Scientific/Technical Coordination Office; 2009 [cited 12 March 2013]. Available from: http://www.sidartha.eu/docs/2007208_SIDARTHa_D5_WP5-

2\%20Report_Coding\%20Manual.pdf.

23. Schrell S, Ziemann A, Garcia-Castrillo Riesgo L, Rosenkötter N, Llorca J, Popa D, et al. Local implementation of a syndromic influenza surveillance system using emergency department data in Santander, Spain. J Public Health (Oxf). 2013;35(3):397-403.

24. Rosenkötter N, Ziemann A, Garcia-Castrillo Riesgo L, Gillet JB, Krafft T, Brand H. Validity and timeliness of syndromic influenza surveillance during the autumn/winter wave of $A(H 1 N 1)$ influenza 2009. Results of emergency medical dispatch, ambulance and emergency department data from three European regions. BMC Public Health. 2013;13:905. doi:10.1186/1471-2458-13-905

25. Buckeridge DL. Outbreak detection through automated surveillance: a review of the determinants of detection. J Biomed Inform. 2007;40(4):370-9.

26. Loveridge $P$, Cooper D, Elliot AJ, Harris J, Gray J, Large S, et al. Vomiting calls to NHS Direct provide an early warning of norovirus outbreaks in hospitals. J Hosp Infect. $2010 ; 74(4): 385-93$.

27. Edge VL, Pollari F, Lim G, Aramini J, Sockett P, Martin SW, et al. Syndromic surveillance of gastrointestinal illness using pharmacy over-the-counter sales. A retrospective study of waterborne outbreaks in Saskatchewan and Ontario. Can J Public Health. 2004;95(6):446-50. 
28. Moore KM, Edgar BL, McGuinness D. Implementation of an automated, real-time public health surveillance system linking emergency departments and health units: rationale and methodology. CJEM. 2008;10(2):114-9.

29. Xing J, Burkom H, Tokars J. Method selection and adaptation for distributed monitoring of infectious dis- eases for syndromic surveillance. J Biomed Inform 2011; 44: 1093-1101.

30. Balter S, Weiss D, Hanson H, Reddy V, Das D, Heffernan R. Three years of emergency department gastrointestinal syndromic surveillance in New York City: what have we found? MMWR Morb Mortal Wkly Rep. 2005;54 Suppl:175-80.

31. Heffernan R, Mostashari F, Das D, Karpati A, Kulldorff M, Weiss D. Syndromic surveillance in public health practice, New York City. Emerg Infect Dis. 2004;10(5):858-64.

32. Andersson T, Bjelkmar P, Hulth A, Lindh J, Stenmark S, Widerstrom M. Syndromic surveillance for local outbreak detection and awareness: evaluating outbreak signals of acute gastroenteritis in telephone triage, web-based queries and overthe-counter pharmacy sales. Epidemiol Infect. 2014;142(2):303-13.

33. Wadl M, Rieck T, Nachtnebel M, Greutelaers B, an der Heiden M, Altmann D, et al. Enhanced surveillance during a large outbreak of bloody diarrhoea and haemolytic uraemic syndrome caused by Shiga toxin/verotoxin-producing Escherichia coli in Germany, May to June 2011. Euro Surveill. 2011;16(24). pii: 19893.

34. Marx MA, Rodriguez CV, Greenko J, Das D, Heffernan R, Karpati AM, et al. Diarrheal illness detected through syndromic surveillance after a massive power outage: New York City, August 2003. Am J Public Health. 2006;96(3):547-53.

35. Steiner-Sichel L, Greenko J, Heffernan R, Layton M, Weiss D. Field investigations of emergency department syndromic surveillance signals--New York City. MMWR Morb Mortal Wkly Rep. 2004;53 Suppl:184-9.

36. Yih WK, Deshpande S, Fuller C, Heisey-Grove D, Hsu J, Kruskal BA, et al. Evaluating real-time syndromic surveillance signals from ambulatory care data in four states. Public Health Rep. 2010;125(1):111-20.

37. Hadler JL, Siniscalchi A, Dembek Z. Hospital admissions syndromic surveillance-Connecticut, October 2001-June 2004. MMWR Morb Mortal Wkly Rep. 2005;54 Suppl:169-73.

38. Horst MA, Coco AS. Observing the spread of common illnesses through a community: using Geographic Information Systems (GIS) for surveillance. J Am Board Fam Med. 2010;23(1):32-41.

39. Greene SK, Huang J, Abrams AM, Gilliss D, Reed M, Platt R, et al. Gastrointestinal disease outbreak detection using multiple data streams from electronic medical records. Foodborne Pathog Dis. 2012;9(5):431-41.

40. Chen D, Cunningham J, Moore K, Tian J. Spatial and temporal aberration detection methods for disease outbreaks in syndromic surveillance systems. Ann GIS. $2011 ; 17(4): 211-20$. 


\section{CHAPTER 4}

\section{Syndromic Surveillance}

Supporting Local Influenza

\section{Surveillance}

published as:

Schrell S, Ziemann A, Garcia-Castrillo Riesgo L, Rosenkötter N, Llorca J, Popa D, Krafft T. Local implementation of a syndromic influenza surveillance system using emergency department data in Santander, Spain. J Public Health (Oxf). 2013;35(3):397-403. 


\section{Abstract}

Background: We assessed the local implementation of syndromic surveillance (SyS) as part of the European project 'System for Information on, Detection and Analysis of Risks and Threats to Health' in Santander, Spain.

Methods: We applied a cumulative sum algorithm on emergency department (ED) chief complaints for influenza-like illness in the seasons 2010-11 and 2011-12. We fine-tuned the algorithm using a receiver operating characteristic analysis to identify the optimal trade-off of sensitivity and specificity and defined alert criteria. We assessed the timeliness of the SyS system to detect the onset of the influenza season.

Results: The ED data correlated with the sentinel data. With the best algorithm settings we achieved $70 / 63 \%$ sensitivity and $89 / 95 \%$ specificity for $2010-11 / 2011-12$. At least 2 consecutive days of signals defined an alert. In 2010-11 the SyS system alerted 1 week before the sentinel system and in 2011-12 in the same week. The data from the ED is available on a daily basis providing an advantage in timeliness compared with the weekly sentinel data.

Conclusions: ED-based SyS in Santander complements sentinel influenza surveillance by providing timely information. Local fine tuning and definition of alert criteria are recommended to enhance validity.

Keywords: communicable diseases, emergency care, epidemiology 


\section{Background}

The European Centre for Disease Prevention and Control (ECDC) estimates that 40 000 premature deaths occur per year in the European Union due to seasonal influenza (1). A comprehensive and effective seasonal influenza surveillance approach can support timely and adequate reactions of professionals and decision-makers during pandemic periods (2). Influenza surveillance should integrate timely surveillance and detection of outbreaks based on different data sources of different specificity as the ECDC suggests in their epidemic intelligence framework. Part of this concept is the real-time data collection from unspecific information sources known as syndromic surveillance (SyS) (3). SyS uses data collected prior to laboratory confirmation with the advantage of providing timelier information on public health threats or sole information on potentially health-threatening events for which no other surveillance information exists (4). SyS understood in such a broad sense can be indicator based (using structured data such as health-care information indicating a certain health condition or disease) and event based (using unstructured information such as media reports to detect or monitor an event) (5).

While there are many initiatives at local, regional and national levels, no European approach to indicator-based SyS exists. The European co-funded project 'System for Information on Detection and Analysis of Risks and Threats to Health' (SIDARTHa) aimed at creating the basis for an emergency medical care data-based SyS approach, which is applicable across Europe. SIDARTHa focuses on emergency data from three sources, prehospital emergency medical dispatch centre call logs, ambulance service run sheets (patient records) and emergency department (ED) patient records. The project followed a local approach to alert and inform local/regional authorities about communicable and non-communicable health threats that are not at all or not timely monitored by existing surveillance systems (6).

This paper presents the results of a case-based assessment of the local implementation of the SIDARTHa SyS approach at the ED of the University Hospital Marque's de Valdecilla (HUMV) in Santander, Spain. We evaluated the system performance in terms of timeliness, sensitivity and specificity for seasonal influenza surveillance.

\section{Methods}

We conducted a retrospective quantitative analysis of two influenza seasons in 2010-11 and 2011-12 with ED data on influenza-like illness (ILI) from the HUMV in Santander, Spain.

\section{Data sets}

With an assigned population catchment of around 300000 inhabitants, the HUMV is the largest hospital serving the Autonomous Region of Cantabria, Spain (7). The SyS system at the HUMV analyses syndromes based on patient's chief complaint codes which are similar to the Canadian Triage and Acuity Scale (8). The chief complaints are assigned only by an emergency physician after a first examination of the patient in the ED. The ILI chief complaint code was defined in accordance with the definition of sentinel general practitioners (GP) in the region who apply the Spanish ILI definition that follows the recommendation of the ECDC (9): 'Sudden onset of symptoms AND at least one of the 
following four systemic symptoms: fever or feverishness, malaise, headache, myalgia AND at least one of the following three respiratory symptoms: cough, sore throat, shortness of breath'. The Spanish authorities added to this definition the point that these symptoms occur without suspicion of any other illness (10). The data set also includes information on age, sex, severity and postal code referring to the patient's residence. The ED data are aggregated daily and automatically uploaded in the SyS system data base during the early hours of the following day. We analysed the ED data on ILI that was available for the period 1 July 2010-30 April 2012.

We compared the syndromic data from the ED with the data from the official influenza surveillance system. The reference data set contained information on the weekly number of ILI cases as reported by sentinel general practices for the Autonomous Region of Cantabria. In Cantabria there are 17 sentinel practices who are voluntarily taking part in the national influenza surveillance system, covering $5.26 \%$ or 30198 inhabitants of the Cantabrian population (10). The sentinel practices were chosen to represent the whole population. No information was available on the population structure of the sentinel ILI patients. ILI cases are defined only by the GP in the sentinel practices. Data were available for Weeks 44/2010-9/2011 and Weeks 44/2011-12/2012. We selected the ED data from the same periods for the comparative analysis. The sentinel system in Cantabria defines a weekly case number of $>74$ per 100000 inhabitants as threshold for high influenza activity (10).

\section{Data analysis}

In a first step, we plotted the time series of the daily and weekly number of ED ILI cases against the reported weekly case numbers of the sentinel system.

As influenza is a rare disease in the ED setting, normal distribution cannot be assumed. We therefore chose a one-sided cumulative sum (CUSUM) algorithm for Poisson distribution as the most suitable aberration detection algorithm for this study (11). We added the fast initial response technique to assure that large chart values do not inflate subsequent ones and control for over-production of out-of-control signals. With this technique a head start of the algorithm is obtained, which tends to give quicker signals (12). The threshold value $\mathrm{h}$ for the CUSUM algorithm was retrieved by a look-up procedure in the table of Lucas (11). All CUSUM calculations were done with Microsoft Excel 2003.

We performed a fine tuning of the CUSUM algorithm by testing different baselines and accepted mean values to obtain optimal surveillance results. Four different scenarios ( $A$, $B, C$ and $D$ ) have been tested with a receiver operating characteristic (ROC) analysis to investigate which scenario would have the best trade-off of sensitivity and specificity and to reduce the number of false-positive signals. We tested if it is suitable to take a baseline mean close to the average of influenza cases outside the influenza season, which is anticipated to be 0 (scenarios $A$ and $B$ ) or rather close to the number of cases occurring outside of the period of high influenza activity (scenarios $C$ and $D$ ). Further, we tested two different accepted means, one close to the baseline mean (scenarios $A$ and $C$ ) and one reflecting the average daily case occurrence during the influenza season (scenarios $B$ and $D$ ). Table 1 lists the variables used for the four scenarios in relation to the different periods analysed in our study and the respective means. The ROC calculations were done using IBM SPSS 15.0. 
The specificity and sensitivity of the CUSUM algorithm were calculated using a daily approach. We compared the signals of the algorithm to the weeks of high influenza activity as reported by the sentinel system. When the threshold rate of the sentinels was exceeded, these weeks were counted as 7 days of expected signals by the CUSUM algorithm. Based on the signal pattern of the CUSUM algorithm we explored criteria for alerts of the SyS system that indicate the onset and end of the high activity influenza period.

\section{Results}

In the two monitored influenza seasons, around 350 ILI cases were counted at the ED per year. During the periods of high influenza activity four to five cases occurred on average per day (around 2\% of all ED cases), while the daily mean during the entire year was 1.1. The majority of ILI patients were middle-aged patients with rather light symptoms. Therefore, no stratification for age or severity was carried out in the syndromic aberration detection analysis.

In both influenza seasons 8 weeks of high influenza activity were identified by the sentinel GPs (Weeks 1-8/2011 and 3-10/2012). On average, 54 cases per week occurred during the periods of high influenza activity in the sentinel system, which is equivalent to 223 cases per 100000 inhabitants. Table 1 summarizes the main characteristics of the ED and sentinel data sets for the different periods used in our study.

The ED data corresponded to the epidemiological curve of reported sentinel cases in both seasons. While the pattern of the epidemiological curve in the season 2011-12 is comparable for the ED and sentinel system data sets, in the season 2010-11 the influenza wave was recognized earlier in the ED than in the sentinel practices (figure 1). In the season 2011-12 the ED case numbers were generally lower than the sentinel case numbers. In the 2010-11 season the weekly numbers of ED ILI cases were higher than the sentinel case numbers in Week 52-2. More ILI patients were visiting the ED in the season 2010-11 (Week 44-9: $n=360$ ) than in the season 2011-12 (Week 44-12: $n=283)$.

\section{Fine tuning, specificity and sensitivity}

The fine-tuning analysis showed the trade-off between the sensitivity and specificity of the different parameters of the CUSUM algorithm indicated by the area under the ROC curve (table 2). In season 2010-11, the most suitable scenario is C with a sensitivity of $70 \%$ and a specificity of $89 \%$. In the season $2011-12$ the optimal trade-off was achieved also by scenario $C$ with a $63 \%$ sensitivity and $95 \%$ specificity (table 2 ). The variables of scenario $C$ were applied in the case study with a threshold value $h$ set to 4 . 
Table 1: Properties of data sets and corresponding variables of fine-tuning scenarios

\begin{tabular}{|c|c|c|c|c|c|c|c|c|}
\hline Period & Total & Mean & $\begin{array}{l}95 \% \text { confidence } \\
\text { interval }\end{array}$ & Median & $\begin{array}{l}\text { Mini- } \\
\text { mum }\end{array}$ & $\begin{array}{l}\text { Maxi- } \\
\text { mum }\end{array}$ & $\begin{array}{l}\text { Standard } \\
\text { deviation }\end{array}$ & CUSUM scenario variables \\
\hline \multicolumn{9}{|l|}{ Daily analysis } \\
\hline Analysis period ${ }^{a}$ & 751 & 1.12 & $0.95-1.30$ & 0 & 0 & 19 & 2.31 & $\begin{array}{l}\text { Accepted mean = } 1 \\
\text { (scenarios A and C) }\end{array}$ \\
\hline Influenza season ${ }^{b}$ & 698 & 1.91 & $1.62-2.21$ & 1 & 0 & 19 & 2.87 & $\begin{array}{l}\text { Accepted mean = } 2 \\
\text { (scenarios B and D) }\end{array}$ \\
\hline $\begin{array}{l}\text { High influenza } \\
\text { activity period }\end{array}$ & 492 & 4.39 & $3.70-5.08$ & 4 & 0 & 19 & 3.67 & \\
\hline $\begin{array}{l}\text { Period outside } \\
\text { influenza season }{ }^{d}\end{array}$ & 53 & 0.17 & $0.12-0.23$ & 0 & 0 & 3 & 0.46 & $\begin{array}{l}\text { Baseline mean }=0.0005 \\
\text { (scenarios } A \text { and } B \text { ) }\end{array}$ \\
\hline $\begin{array}{l}\text { Period outside high } \\
\text { influenza activity } \\
\text { periode }^{\text {e }}\end{array}$ & 259 & 0.46 & $0.38-0.55$ & 0 & 0 & 7 & 1.06 & $\begin{array}{l}\text { Baseline mean }=0.5 \\
\text { (scenarios } C \text { and } D \text { ) }\end{array}$ \\
\hline
\end{tabular}

Weekly analysis ${ }^{f}$

\begin{tabular}{lccccccc} 
ED & 633 & 16.23 & $10.52-21.94$ & 11 & 0 & 74 & 17.61 \\
Sentinel GPs & 1003 & 25.72 & $16.19-35.24$ & 12 & 0 & 105 & 29.38 \\
\hline
\end{tabular}

a 1 July 2010-30 April 2012

b 1 October - 31 March 2010-11 + 1 October - 31 March 2011-12

c 3 January -27 February $2011+16$ January - 11 March 2012

d 1 July - 30 September $2010+1$ April - 30 September $2011+1$ April - 30 April 2012

e 1 July 2010 - 2 January 2011 + 28 February 2011 - 15 January $2012+12$ March - 30 April 2012

f weeks 44/2010 - 9/2011+44/2011-12/2012

Table 2: Results of fine-tuning scenarios

\begin{tabular}{lllllll} 
Scenario & Period & $\begin{array}{l}\text { Sensitivity } \\
(\%)\end{array}$ & Specificity (\%) & $\begin{array}{l}\text { Positive predictive } \\
\text { value (\%) }\end{array}$ & $\begin{array}{l}\text { Negative predictive } \\
\text { value (\%) }\end{array}$ & $\begin{array}{l}\text { Area under } \\
\text { ROC curve }\end{array}$ \\
\hline A & $2010-11$ & 75 & 80 & 75 & 80 & 0.775 \\
& $2011-12$ & 64 & 86 & 73 & 80 & 0.750 \\
B & $2010-11$ & 75 & 81 & 76 & 80 & 0.782 \\
& $2011-12$ & 64 & 89 & 78 & 80 & 0.766 \\
C & $2010-11$ & 70 & 89 & 83 & 79 & 0.791 \\
& $2011-12$ & 63 & 95 & 88 & 80 & 0.785 \\
& $2010-11$ & 66 & 91 & 86 & 77 & 0.778 \\
D & $2011-12$ & 50 & 96 & 88 & 76 & 0.728 \\
\hline
\end{tabular}



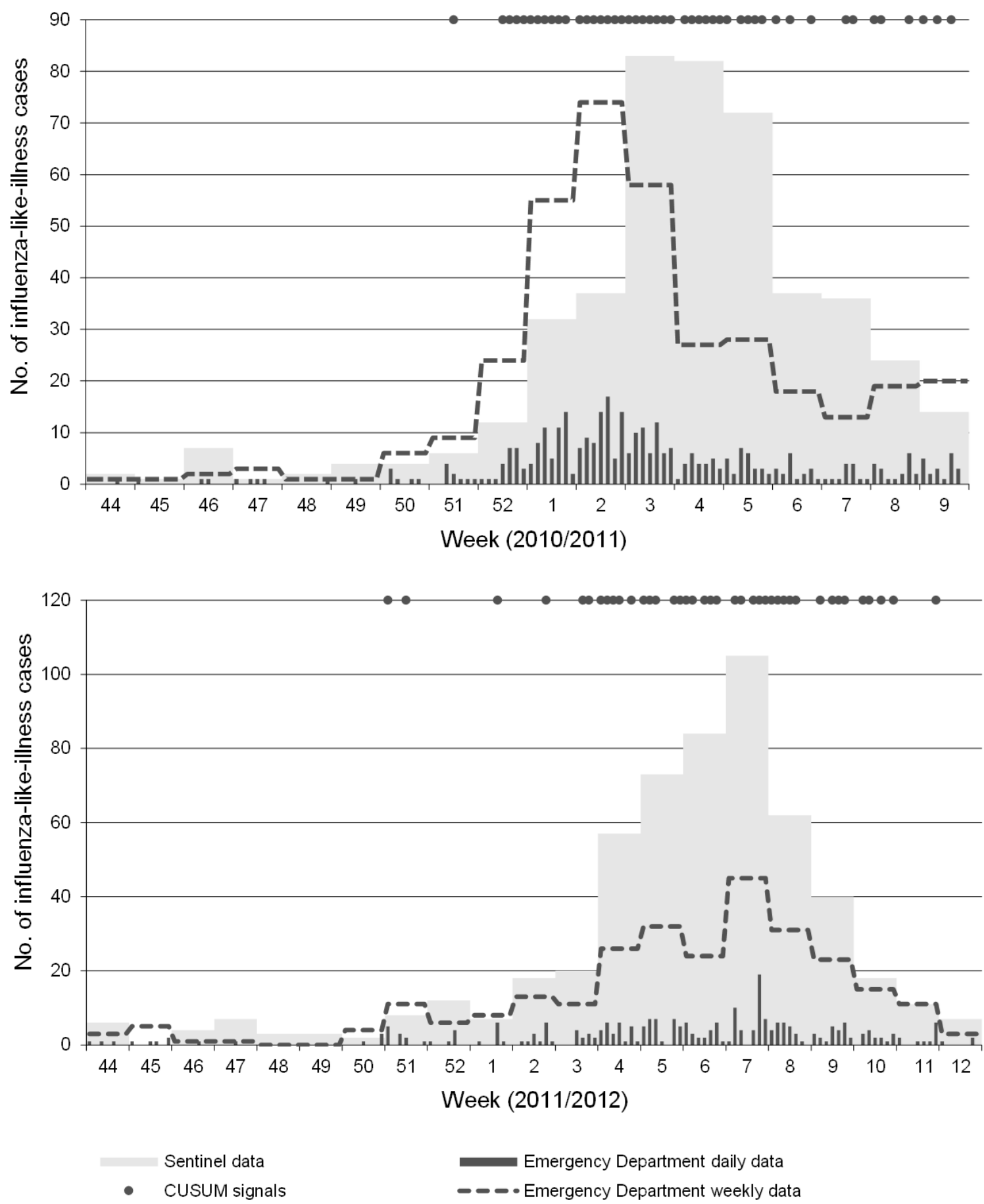

Figure 1: Time series of daily and weekly ED ILI cases from Santander, Spain compared with weekly sentinel GP ILI cases for the region of Cantabria, Spain for the influenza seasons 2010-11 and 2011-12 and respective CUSUM algorithm signals

\section{Alert criteria}

On average there have been five signals of the CUSUM algorithm per week during the periods of high influenza activity. Before and after these periods only sporadic signals occurred, none with consecutive days of signals (figure 1). Based on this pattern we defined the criterion for alert of the SyS system for ILI as at least 2 consecutive days of CUSUM signals. 


\section{Timeliness}

Applying the alert criterion the SyS system produced the first alert in the season 2010111 week before the sentinel system reached the threshold for high influenza activity (four signals on 4 consecutive days in Week 52). In 2011-12 the first alert is produced in the same week in which the sentinels reached the threshold (two signals on 2 consecutive days in Week 3) (figure 1). Compared with the weekly reported sentinel data timelier information is reported by the ED data-based system which reports daily.

\section{Discussion}

\section{Main findings of this study}

Daily SyS of ILI based on the ED data in Santander, Spain correlated with the outbreak pattern reported by the regional sentinel system.

A one-sided CUSUM algorithm for Poisson distributed data with the parameters of 0.5 for the baseline and 1 as the accepted mean was identified as best setting for the local analysis. We defined a local alert criterion of at least 2 consecutive days of CUSUM signals for detecting the onset and the end of the influenza season.

The SyS system indicated the onset of the period of high influenza activity in one season 1 week before and in the other season at the same time as the sentinel system. The daily reported ED data provide an earlier warning compared with the weekly sentinel reporting.

\section{What is already known on this topic?}

SyS is often used to detect influenza especially based on the ED data (13). Buehler et al. describe ED data-based surveillance as 'common extension' to the surveillance of seasonal influenza based on sentinel GPs (14). Review results suggest general usefulness of SyS for timely and sensitive information of outbreaks affecting large parts of the population such as influenza. But the performance of the systems varies depending on, for example, the aberration detection algorithm applied (15). As Griffin et al. suggest, fine-tuning of algorithms can be useful to enhance the performance of a SyS system (16).

\section{What this study adds}

The Poisson CUSUM algorithm was useful to detect seasonal influenza in Santander, Spain, in combination with fine tuning of parameters and definition of an alert criterion. The alert criterion reduced the number of false alerts based on single CUSUM signals and supported the timely detection of the onset and end of the high influenza activity period. Some other SyS systems apply the same alert criterion of consecutive days of signals. Further criteria explored by these SyS systems are the magnitude of the aberration, the age and severity of cases, and the activity of other syndromes and other SyS system sites (17-19). The magnitude of the aberration would not have added precision in terms of defining alerts in our case study. No significant differences in age and severity were found in our ED ILI data set, which might be due to the relatively small sample size. The criterion of signals in other syndrome groups could be a future option. Especially, the comparison to the syndrome group of respiratory illness that is established in the SyS system in Santander could be of value. Also the criterion of signal activity in neighbouring 
SyS sites could be explored in the future if further SyS systems would be implemented in Northern Spain. Our alert criterion needs to be assessed in the everyday work of the SyS system in future influenza seasons.

Our daily approach of calculating sensitivity and specificity provides a more precise picture of the performance of the SyS system. Other studies aggregate daily syndromic data to weeks for comparison to weekly reference data $(20,21)$. Ansaldi et al. compared the ED data with the sentinel GP data following a daily approach and reached a similar specificity and sensitivity using a 5-day moving average algorithm (17). In general, it is difficult to compare validity between different SyS systems as factors, such as the chosen statistics/algorithms, represented population and outbreak characteristics, are influencing the performance (15).

In one of the seasons the ED data-based SyS system indicated the beginning of the influenza season 1 week earlier than the sentinel system. In the other season both data sources showed elevated influenza activity in the same week. This is in line with the results of other SyS systems based on the ED data that indicated a similar or earlier onset of 1-3 weeks for influenza compared with traditional surveillance data sources depending on the reference data sources used and statistical measures applied (13). The application of the same case definition in the two systems in Cantabria makes it possible to more precisely determine the early detection effect of the ED data compared with the sentinel GP data. This could be a reflection of the treatment-seeking behaviour in Spain that is characterized by a high number of patients utilizing EDs with conditions that would more appropriately be treated in a primary care setting (22).

For the season 2010-11, the health system organization and treatment-seeking behaviour of the population is also the likely explanation for the earlier wave seen in the ED. The period of high influenza activity began during the Spanish Christmas holidays (26 December-10 January) when most GP practices were closed and patients visited the ED instead (23). This highlights the usefulness of an ED-based surveillance system that is continuously operating during the entire year while the sentinel system based on GP practices is not. The beginning of the influenza season in season 2010-11 could only be detected in near real time by the ED SyS system. This suggests that ED data-based SyS can constitute an important supplement to the surveillance system to get a more complete picture of the influenza disease burden on the population. However, for general seasonal influenza surveillance, it needs to be taken into account that the populations visiting GPs and the ED are usually reflecting different populations, i.e. the general population, and more severe and acute, out-of-hours cases, respectively.

The influenza season 2010-11 in Santander also highlights the pressure of a high number of influenza patients on the resources of the ED, especially during holiday seasons when other health-care providers are closed $(24,25)$. The implementation of a daily automated Sys system with locally adjusted algorithms and alert thresholds supports the decisionmakers in the hospital to timely adjust their resources to the actual demand.

Once implemented, an automated SyS system can be a stable and flexible tool for timely monitoring of various public health events. Such a generic surveillance system can rapidly be applied during unexpected crisis situations or out-of-season epidemics such as the influenza pandemic in 2009 (26, 27). The Department of Health of the Autonomous Region of Cantabria officially endorsed the integration of ED data-based SyS as implemented in Santander as a supplementary surveillance information source in the region. 


\section{Limitations of this study}

The time period of two seasons of influenza is quite short for a representative assessment. Furthermore, the analysis has been conducted retrospectively. A prospective analysis also of the experience of the regional public health authority during influenza seasons can bring further insight in the future.

The objectivity of physicians in diagnosing ILI could be influenced by the higher expectation of influenza case occurrence during the influenza season as suggested by Moore et al. (28). We anticipate the multiple aspect case definition used in Santander to reduce false diagnoses to a minimum.

We applied one aberration detection algorithm that is used in other syndromic influenza surveillance systems and that is easy to implement but there are others that are equally often used such as regression analysis or moving averages (15). A statistical modelling of time series data with seasonal autoregressive moving average (SARIMA) algorithms could be suitable to our context as seasonal patterns and trends can be accounted for in a more precise manner (29).

The spatial distribution and spread of influenza can yield additional information for decision-making during an epidemic. Other studies have shown that syndromic data can be scanned for space-time clusters. These studies focused on large areas such as New York City, England or the Netherlands consisting of a large number of small-area postal code regions as basis for the analysis (30-32). Santander covers a comparatively small area with 12 postal codes for which we considered a spatial analysis not to provide an added value. This assumption could change in the future if the SyS system is enlarged to cover neighbouring areas in addition.

\section{Conclusions}

This case study shows the added value of SyS based on routine ED data to effectively complement sentinel surveillance for seasonal influenza at the local level in Spain. We recommend the local adjustment of the aberration detection algorithms and the definition of alert criteria per syndrome and data source to improve the performance of a SyS system.

\section{Acknowledgements}

The authors would like to thank the Instituto de Salud Carlos III for providing the reference data from sentinel GPs for the Autonomous Region of Cantabria.

This work was supported by the European Union, in the framework of the Public Health Programme (grant number 2007208). 


\section{References}

1. European Centre for Disease Prevention and Control. The hard facts are often overlooked: seasonal influenza remains a threat; 2010 [cited 18 June 2012]. Available from: http://ecdc.europa.eu/en/healthtopics/ seasonal_influenza/key_messages/Pages/key_messages_2.aspx.

2. European Centre for Disease Prevention and Control. Annual epidemiological report 2011-reporting on 2009 surveillance data and 2010 epidemic intelligence data. Stockholm: European Centre for Disease Prevention and Control; 2011.

3. Paquet C, Coulombier D, Kaiser R, Ciotti M. Epidemic intelligence: a new framework for strengthening disease surveillance in Europe. Euro Surveill. 2006;11(12):212-4.

4. Triple S Project. Assessment of syndromic surveillance in Europe. Lancet. 2011;378(9806):1833-4.

5. Amato-Gauci A, Ammon A. The surveillance of communicable diseases in the European Union-a long-term strategy (2008-2013). Euro Surveill. 2008;13(26):pii: 18912.

6. Ziemann A, Krafft T, Garcia-Castrillo Riesgo L, Fischer M, Kraemer A, Lippert F, et al. The SIDARTHa approach-European emergency data-based syndromic surveillance. Eur J Public Health. 2009;19(Suppl. 1):73.

7. Hospital Universitario Marque's de Valdecilla (HUMV). Admisio'n de enfermos [Internet]; 2007 [cited 18 June 2012]. Available from: http://www.humv.es/index.php?option=com_content\&task=view\&id=24\&Itemi $d=117$.

8. Bullard MJ, Unger B, Spence J, Grafstein E, CTAS National Working Group. Revisions to the Canadian Emergency Department Triage and Acuity Scale (CTAS) adult guidelines. CJEM. 2008;10(2):136-51. Erratum in: CJEM. $2008 ; 10(4): 330$.

9. European Centre for Disease Prevention and Control. Influenza case definition [Internet]; 2012 [cited 14 January 2013]. Available from: http://ecdc.europa.eu/en/activities/surveillance/eisn/surveillance/pages/influen za_case_definitions.aspx.

10. Instituto de Salud Carlos III. Vigilancia de gripe en Espan ${ }^{a}$. Informacio'n adicional- Temporada 2011-2012; 2012 [cited 18 June 2012]. Available from: http://vgripe.isciii.es/gripe/documentos/20112012/home/Informacion_adicional _SVGE_temporada2011-12_v8.pdf.

11. Lucas JM. Counted data CUSUM's. Technometrics. 1985;27(2): 129-44.

12. Lucas JM, Crosier RB. Fast initial response for CUSUM qualitycontrol schemes: Give your CUSUM a head start. Technometrics. 1982;24(3):199-205.

13. Dailey $L$, Watkins RE, Plant AJ. Timeliness of data sources used for influenza surveillance. J Am Med Inform Assoc. 2007;14(5):626-31.

14. Buehler JW, Whitney EA, Smith D, Prietula MJ, Stanton SH, Isakov AP. Situational uses of syndromic surveillance. Biosecur Bioterror. 2009;7(2):165-77. 
15. Buckeridge DL. Outbreak detection through automated surveillance: a review of the determinants of detection. J Biomed Inform. 2007;40(4):370-9.

16. Griffin BA, Jain AK, Davies-Cole J, Glymph C, Lum G, Washington SC, et al. Early detection of influenza outbreaks using the DC Department of Health's syndromic surveillance system. BMC Public Health. 2009;9:483. doi: 10.1186/1471-24589-483.

17. Ansaldi F, Orsi A, Altomonte F, Bertone G, Parodi V, Carloni R, et al. Emergency department syndromic surveillance system for early detection of 5 syndromes: a pilot project in a reference teaching hospital in Genoa, Italy. J Prev Med Hyg. 2008;49(4):131-5.

18. Riccardo F, Napoli C, Bella A, Rizzo C, Rota MC, Dente MG, et al. Syndromic surveillance of epidemic-prone diseases in response to an influx of migrants from North Africa to Italy, May to October 2011. Euro Surveill. 2011;16(46): pii: 20016.

19. Smith $G E$, Cooper $D L$, Loveridge $P$ et al. A national syndromic surveillance system for England and Wales using calls to a telephone helpline. Euro Surveill. $2006 ; 11(12): 220-4$.

20. May LS, Griffin BA, Bauers NM, Jain A, Mitchum M, Sikka N, et al. Emergency department chief complaint and diagnosis data to detect influenza-like illness with an electronic medical record. West J Emerg Med. 2010; 11(1):1-9.

21. Liljeqvist GT, Staff M, Puech M, Blom H, Torvaldsen S. Automated data extraction from general practice records in an Australian setting: trends in influenza-like illness in sentinel general practices and emergency departments. BMC Public Health. $2011 ; 11: 435$.

22. Garcia-Armesto S, Abadia-Taira MB, Duran A, Hernandez-Quevedo C, BernalDelgado E. Spain: health system review. Health Syst Transit. 2010;12(4):1-295.

23. Salazar A, Corbella X, Sanchez JL, Argimon JM, Escarrabill J. How to manage the ED crisis when hospital and/or ED capacity is reaching its limits. Report about the implementation of particular interventions during the Christmas crisis. Eur J Emerg Med. 2002;9(1):79-80.

24. Hoot NR, Aronsky D. Systematic review of emergency department crowding: causes, effects, and solutions. Ann Emerg Med. 2008;52(2):126-36.

25. Silka PA, Geiderman JM, Goldberg JB, Kim LP. Demand on ED resources during periods of widespread influenza activity. Am J Emerg Med. 2003;21(7):534-9.

26. Rosenkötter N, Kraan J, Ziemann A, Castrillo L, Vergeiner G, Meulemans A, et al. Pandemic influenza 2009-performance of the emergency medical data-based syndromic surveillance system SIDARTHa. Eur J Public Health. 2010;20(Suppl. 1):81-82.

27. Savage R, Chu A, Rosella LC, Crowcroft NS, Varia M, Policarpio ME, et al. Perceived usefulness of syndromic surveillance in Ontario during the H1N1 pandemic. J Public Health. 2012;34(2):195-202. 
28. Moore K, Black J, Rowe S, Franklin L. Syndromic surveillance for influenza in two hospital emergency departments. Relationships between ICD-10 codes and notified cases, before and during a pandemic. BMC Public Health. 2011;11:338. doi: $10.1186 / 1471-2458-11-338$

29. Craigmile PF, Kim N, Fernandez SA, Bonsu BK. Modeling and detection of respiratory-related outbreak signatures. BMC Med Inform Decis Mak. 2007; 7:28.

30. Heffernan R, Mostashari F, Das D, Karpati A, Kulldorff M, Weiss D. Syndromic surveillance in public health practice, New York City. Emerg Infect Dis. 2004;10:858-64.

31. Cooper DL, Smith GE, Regan M, Large S, Groenewegen PP. Tracking the spatial diffusion of influenza and norovirus using telehealth data: a spatiotemporal analysis of syndromic data. BMC Med. 2008;6:16. doi: 10.1186/1741-7015-616.

32. van den Wijngaard CC, van Asten L, van Pelt W, Doornbos G, Nagelkerke NJ, Donker GA. Syndromic surveillance for local outbreaks of lower-respiratory infections: would it work? PLoS One. 2010;5:e10406. doi: 10.1371/journal. pone.0010406 



\section{CHAPTER 5}

\section{The Added Value of Syndromic Surveillance for Implementing the International Health Regulations (2005)}

published as:

Ziemann A, Rosenkötter N, Garcia-Castrillo Riesgo L, Fischer M, Krämer A, Lippert FK, Vergeiner G, Brand H, Krafft T. Meeting the International Health Regulations (2005) surveillance core capacity requirements at the subnational level in Europe: the added value of syndromic surveillance. BMC Public Health. 2015;15:107. doi: 10.1186/s12889015-1421-2. 


\section{Abstract}

Background:

The revised World Health Organization's International Health Regulations (2005) request a timely and all-hazard approach towards surveillance, especially at the subnational level. We discuss three questions of syndromic surveillance application in the European context for assessing public health emergencies of international concern: (i) can syndromic surveillance support countries, especially the subnational level, to meet the International Health Regulations (2005) core surveillance capacity requirements, (ii) are European syndromic surveillance systems comparable to enable cross-border surveillance, and (iii) at which administrative level should syndromic surveillance best be applied?

Discussion:

Despite the ongoing criticism on the usefulness of syndromic surveillance which is related to its clinically nonspecific output, we demonstrate that it was a suitable supplement for timely assessment of the impact of three different public health emergencies affecting Europe. Subnational syndromic surveillance analysis in some cases proved to be of advantage for detecting an event earlier compared to national level analysis. However, in many cases, syndromic surveillance did not detect local events with only a small number of cases.

The European Commission envisions comparability of surveillance output to enable crossborder surveillance. Evaluated against European infectious disease case definitions, syndromic surveillance can contribute to identify cases that might fulfil the clinical case definition but the approach is too unspecific to comply to complete clinical definitions. Syndromic surveillance results still seem feasible for comparable cross-border surveillance as similarly defined syndromes are analysed.

We suggest a new model of implementing syndromic surveillance at the subnational level. In this model, syndromic surveillance systems are fine-tuned to their local context and integrated into the existing subnational surveillance and reporting structure. By enhancing population coverage, events covering several jurisdictions can be identified at higher levels. However, the setup of decentralised and locally adjusted syndromic surveillance systems is more complex compared to the setup of one national or local system.

\section{Summary:}

We conclude that syndromic surveillance if implemented with large population coverage at the subnational level can help detect and assess the local and regional effect of different types of public health emergencies in a timely manner as required by the International Health Regulations (2005).

Keywords: Public health surveillance, Europe, World Health Organization 


\section{Background}

\section{Diverse health threats and implementation of the International Health Regulations (2005) in Europe}

Europe faces different health threats which are arising from infectious disease outbreaks, natural disasters or man-made events. The variety of potential health threats led the World Health Organization (WHO) to adjust their International Health Regulations (IHR (2005)). The IHR (2005) now follow an all-hazard approach, focusing on an "illness or medical condition, irrespective of origin or source, that presents or could present significant harm to humans" (1). Detection, assessment and immediate reporting play an important role in the IHR (2005) and Article 5 requests every member state, within five years after the regulations came into force, to have established appropriate surveillance and response capacities (1). Until now, surveillance in Europe primarily follows the approach of specific notifiable communicable disease reporting, which does not foresee detection or assessment of other health threats which are not defined as notifiable diseases (2). Generally, notifiable communicable disease reporting provides data on a weekly basis. Timelier information and information on different kinds of health threats are difficult to retrieve because separate public health surveillance systems cannot be established for every kind of threat. As a result, about one third of WHO Europe member states asked for an extension of the compliance date for implementing the core capacities for public health surveillance and response with the new all-hazard requirement being seen as an obstacle (3).

\section{Public health surveillance in Europe - a matter of comparability}

For the European Union (EU), comparability of surveillance results in the form of comparable case definitions, data formats and diagnostic codes is of high value to enable cross-border monitoring of events. EU member states, however, are very diverse and adopting comparative case definitions based on similar data sources is difficult to achieve (4). The new directive on serious cross-border health threats 1082/2013/EU acknowledges the IHR (2005) requirements and strengthens the Union's mandate to coordinate in times of public health crises which are potentially concerning more than one member state (5). For communicable disease surveillance, the directive requires member states to provide information to the EU on the progression of an outbreak and about any unusual phenomena and outbreaks of unknown origin. The EU provides case definitions for comparable reporting of cases in implementing decision 2012/506/EU which member states have to use (6). These are based on a classification of cases based on clinical signs and symptoms, laboratory, and epidemiological characteristics enabling the identification of possible, probable and confirmed cases. For threats of biological origin consisting of bio toxins or other harmful biological agents not related to communicable diseases and threats of chemical, environmental or unknown origin, member states shall inform each other based on the information from their own surveillance systems. However, the EU may adopt case definitions to which member states shall adhere (5). The question is how member states' surveillance capacity in terms of the IHR (2005) can be strengthened against the backdrop of both, European comparability requirements and European diversity. 


\section{Role of the subnational level for surveillance in Europe}

We understand the subnational level in this paper as primary level, e.g., county, and intermediate level, e.g., province, of the public health response in a country according to the IHR (2005) (1). The subnational level is often the first to identify a health threat and has the responsibility to inform higher levels about an event. Also the response to an event is starting at the subnational level (7). The IHR (2005) explicitly request the responsibility of the primary and intermediate public health response level to detect events in the whole state territory and for immediately assessing and reporting information on such events to higher levels (1).

But how can the subnational level be equipped with the means to detect different types of events or related health effects in a timely fashion? Local intelligence in the form of professionals reporting on events, e.g., in schools, or health care institutions, are the cornerstone of detecting the onset of events at the local level, as shown for example, during the $2009 \mathrm{~A} / \mathrm{H} 1 \mathrm{~N} 1$ pandemic $(8,9)$. Still, there is a risk to overlook patterns that might be detected only when taking a wider perspective beyond the patients treated by one clinician or in one institution $(10,11)$. Surveillance systems that pool information from multiple institutions or jurisdictions can potentially detect events that are not represented in the data of any single region or institution. Using routine data, for instance from health care services, for timely detection and assessment of public health threats is in principle the idea behind the approach of syndromic surveillance.

\section{Syndromic surveillance - a means to meet the IHR (2005) surveillance requirements?}

According to a recent definition by the European project Triple S-AGE, syndromic surveillance augments traditional surveillance systems by providing clinically nonspecific but (near) real-time information on the public health impact of events, gained from existing and if possible automatically generated data that were originally not collected for surveillance purposes (12). The Triple S-AGE project inventoried syndromic surveillance systems in Europe and provided a detailed overview on their characteristics (13). The project identified 124 syndromic surveillance systems worldwide and 60 in Europe (13, 14). Syndromic data sources are ranging from nonclinical sources such as web search logs, medications sales registries, and telephone helpline call logs to clinical sources such as chief complaints from primary care or emergency departments, and veterinary records (14).

Timeliness, flexibility and cost-effectiveness are considered to be the major strengths of syndromic surveillance which could make it a suitable solution for gaining timely information on different kinds of health threats as required by the IHR (2005) (15). Its major weakness is the lack of specificity which can lead to false alerts and undetected events (16). Therefore, the usefulness of syndromic surveillance is not undisputed, although the approach has been applied for over a decade, using different kinds of data sources and targeting various health threats (16-18). The WHO evaluated syndromic surveillance as not applicable to become part of a global regulation to support countries to meet the IHR (2005) requirements but it was rated as potentially useful to support surveillance within countries (19). This assessment was done back in 2001 . We think it is time for a reassessment based on the evidence collated since then, and to consider a 
reinforced endorsement of syndromic surveillance for the support of countries' surveillance capacity as required by the IHR (2005).

Below, we discuss the following questions around the usefulness of syndromic surveillance in the light of the IHR (2005) implementation in Europe:

1. How can syndromic surveillance be used to support European countries to meet the IHR (2005) requirements for the core capacity of immediate detection, assessment and reporting of different kinds of (potential) Public Health Emergencies of International Concern (PHEIC), especially at the subnational level?

2. How comparable are syndromic surveillance systems to enable cross-border surveillance in Europe referring to case definitions, data formats and diagnostic coding systems?

3. What are strengths and weaknesses of different implementation models for subnational syndromic surveillance in Europe?

\section{Discussion}

\section{Contribution of syndromic surveillance to support European countries to meet the IHR (2005) requirements}

In the following, we explore three European public health emergencies during which syndromic surveillance was applied, the 2009 A/H1N1 influenza pandemic, the volcanic ash plume which covered Europe in 2010, and the 0104: $\mathrm{H} 4$ gastrointestinal outbreak in 2011. The pandemic was declared a PHEIC in the framework of the IHR (2005) while the other two events were reported to WHO by member states as potential PHEIC. We analyse the contribution of syndromic surveillance in the three cases in terms of timeliness, added value of subnational level application and flexibility in terms of an all-hazard approach.

\section{Purpose of syndromic surveillance during three public health emergencies}

During the $\mathrm{A} / \mathrm{H} 1 \mathrm{~N} 1$ pandemic, the purpose of the application of syndromic surveillance was to detect the onset of the pandemic in a country and to gain timely information on the spatial and temporal development. Next to the syndromic surveillance systems listed in table 1 and table 2a, we identified seven additional systems, for which no details were retrievable and they were not included in further analysis $(13,20)$.

During the time in which the volcanic ash plume covered Europe in April 2010, syndromic surveillance was used to timely assess if there was a public health impact of the plume or rather to provide reassurance that the plume had no health effect. Next to the syndromic surveillance systems listed in table 3, further syndromic surveillance systems were reported in Iceland and France for which we could not find any further details (39). None of the systems produced a syndromic signal which could be connected to the ash plume. This was in line with the conclusions by WHO and the European Centre for Disease Prevention and Control $(\operatorname{ECDC})(39,40)$. During this public health emergency, syndromic surveillance systems were the only source of information to measure any direct health impact.

The 0104:H4 outbreak in 2011 originated in Northern Germany and later affected 13 countries in Europe, Canada and the United States of America. The outbreak first 
developed unnoticed for around two weeks mainly because of time lost in the information flow from local to regional and national level in the decentralised epidemiological reporting system in Germany (43). Upon becoming aware of the outbreak, the German national centre for disease control enhanced the frequency of reporting from subnational to national level to daily reporting and implemented a syndromic surveillance system for bloody diarrhoea in emergency departments (44). We did not identify reports of syndromic surveillance use in any of the other affected countries during this public health emergency.

\section{Timeliness of syndromic surveillance during three public health emergencies}

During the influenza pandemic, the syndromic surveillance systems provided information on the onset or peak of the pandemic on average half a week before traditional surveillance systems, at the earliest (table 2). These timeliness assessments did not take into account the common reporting delay of traditional sentinel influenza surveillance systems. They refer to an earlier detection of cases analysing data sources in which (potential) influenza patients are registered earlier in the course of illness or treatment, as compared to traditional data collected later in the process by sentinel general practitioners or laboratories. Additional time is gained because syndromic surveillance information usually is available daily while sentinel and laboratory information often is reported up to a week after the case was registered $(21,23,35)$. Many syndromic systems were already established and were ready to be used when the pandemic occurred which saved time in comparison to ad-hoc set up systems.

During the volcanic ash plume event, information was made available to the English public one day after the first day of eruption and two days afterwards in Scotland. Information was updated in the following days and weeks (42). The first information from the systems in Austria, Germany, and Spain was available to the public two weeks after the first day of eruption and was updated again 10 days later (41) (table 1). There is no information on the timeliness for the syndromic surveillance system in Sweden. As there were no other health surveillance systems used during the ash plume, there is no gold standard to compare the timeliness of syndromic surveillance systems to.

During the 0104:H4 outbreak, the syndromic surveillance system was implemented on 27 May, 8 days after Robert Koch-Institute was notified of a first cluster of cases and 5 days after the EU and WHO were informed. The system was terminated on 30 September 2011. Syndromic reporting was daily. The syndromic surveillance system was set up adhoc, relied on manual data collection and reporting (via fax or email) and was voluntary. Thus, syndromic reporting varied in completeness and continuity (43).

In synthesis, syndromic surveillance provided timely information during the three events supporting the IHR (2005) requirement of immediate assessment and reporting. Also during other subnational and national emergencies, syndromic surveillance provided timelier information, as for example found in a review by Dailey et al. (45). For rare or non-communicable health threats such as the volcanic ash plume, there are often no established health surveillance systems. As clinically specific systems cannot be implemented for every conceivable health threat like the volcanic ash plume, syndromic surveillance is often the only source of timely information (46). 


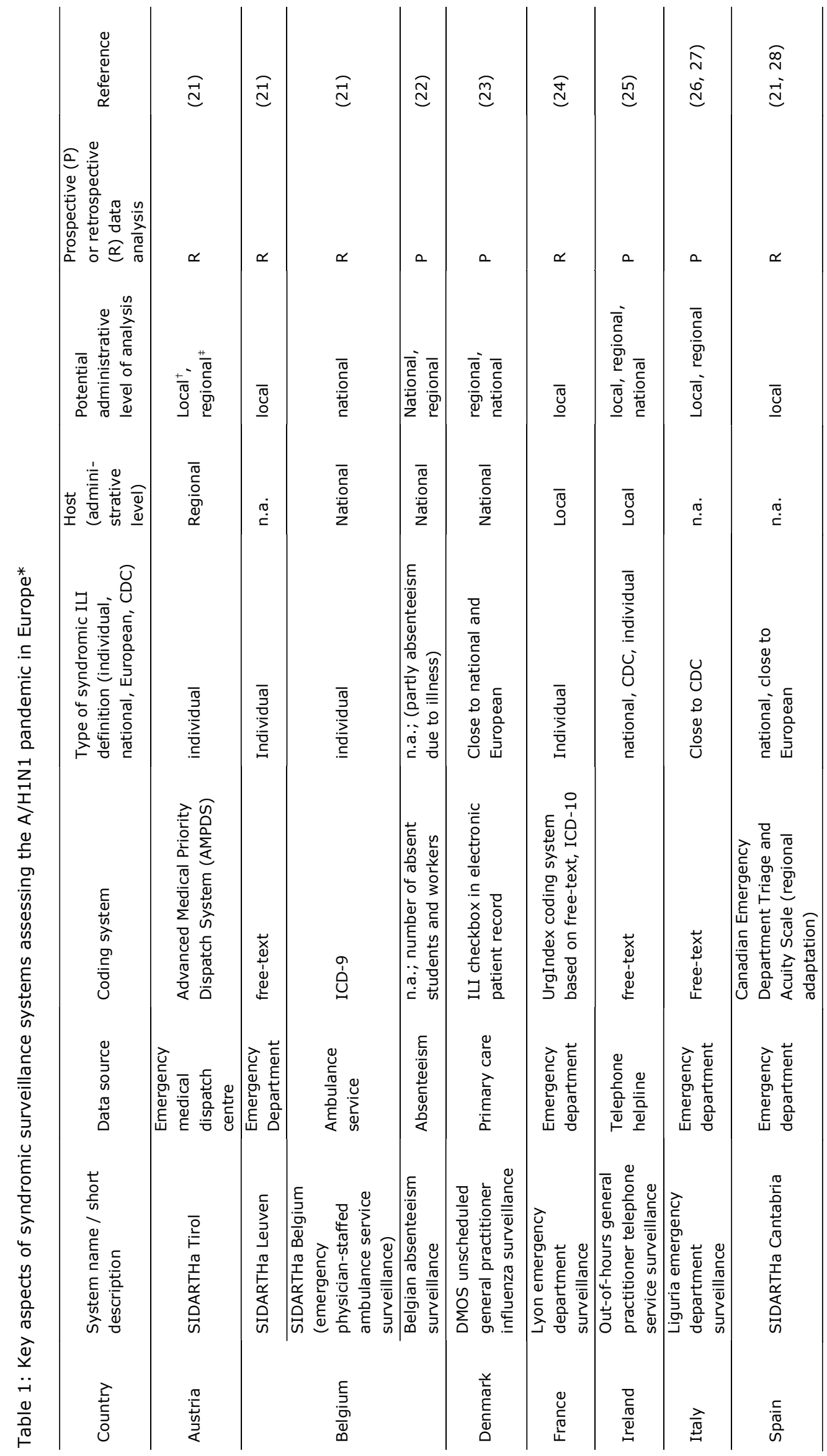




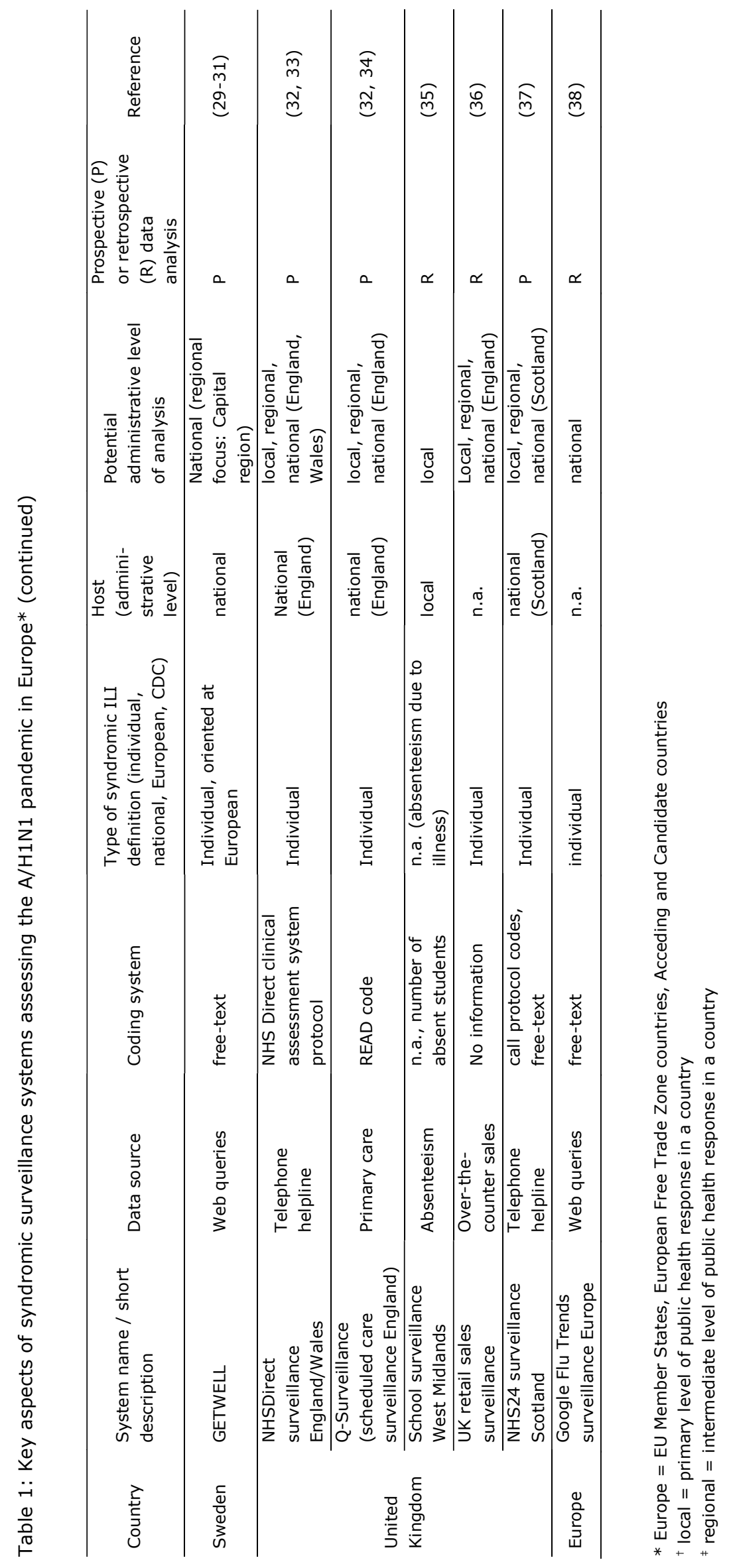




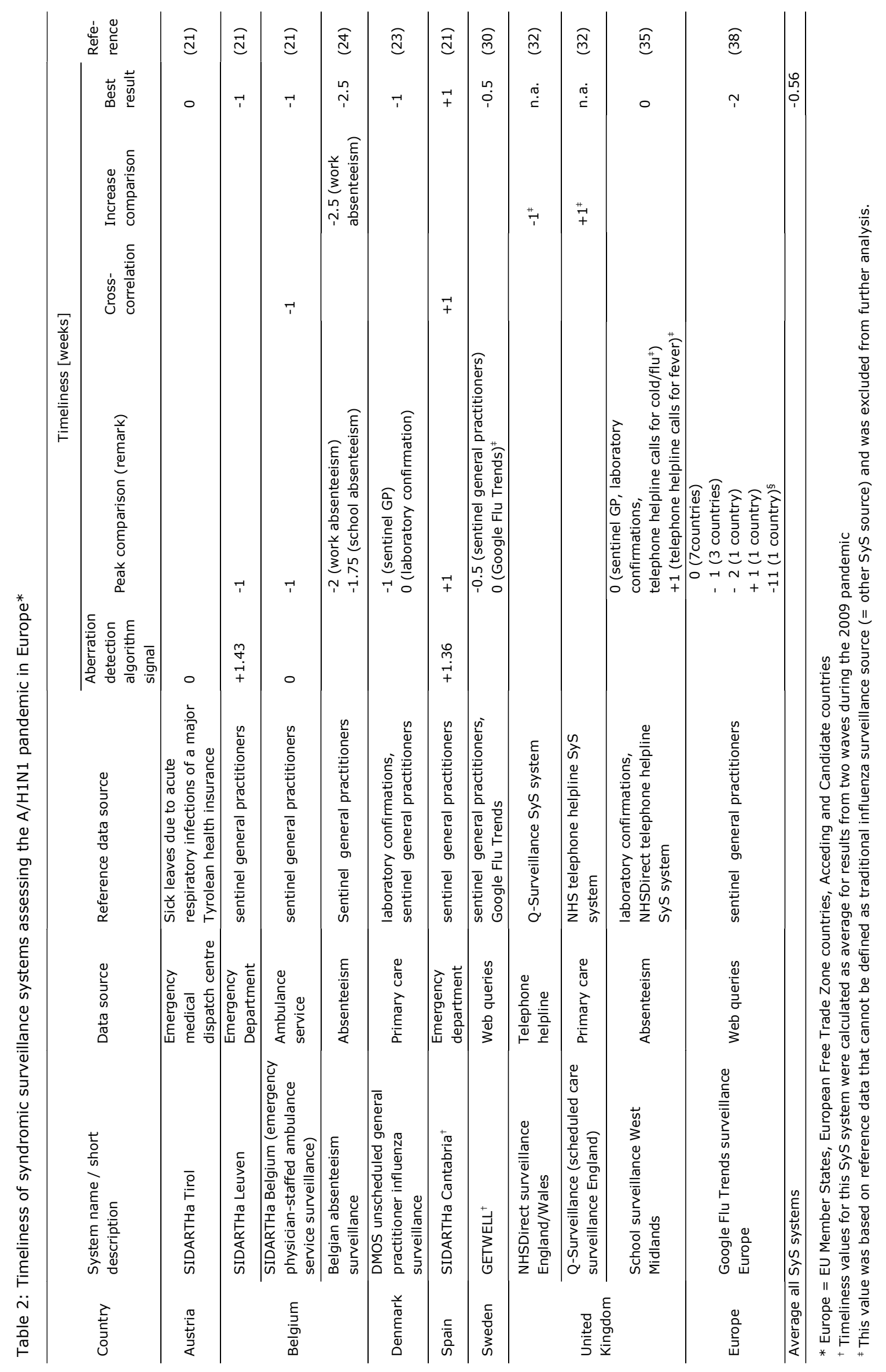


Table 3: Key aspects of syndromic surveillance systems assessing the volcanic ash plume in Europe*

\begin{tabular}{|c|c|c|c|c|c|c|c|}
\hline Country & $\begin{array}{l}\text { System name / } \\
\text { short description }\end{array}$ & Data source & Syndromes & $\begin{array}{l}\text { Time- } \\
\text { liness } \\
\text { [days] }^{\dagger}\end{array}$ & $\begin{array}{l}\text { Sub-national } \\
\text { level appli- } \\
\text { cation } \\
(\text { Yes }=\text { Y; } \\
\text { No=N) }\end{array}$ & $\begin{array}{l}\text { Pros- } \\
\text { pective }(P) \\
\text { or retro- } \\
\text { spective } \\
(\mathrm{R}) \text { data } \\
\text { analysis }\end{array}$ & $\begin{array}{l}\text { Refe- } \\
\text { rence }\end{array}$ \\
\hline Austria & SIDARTHa-Tirol & $\begin{array}{l}\text { Emergency } \\
\text { medical } \\
\text { dispatch }\end{array}$ & $\begin{array}{l}\text { Respiratory } \\
\text { syndrome, } \\
\text { cardiovascular } \\
\text { syndrome, traffic- } \\
\text { related injuries }\end{array}$ & 14 & $Y$ & $\mathrm{R}$ & $(41)$ \\
\hline Germany & $\begin{array}{l}\text { SIDARTHa- } \\
\text { Göppingen }\end{array}$ & $\begin{array}{l}\text { Ambulance } \\
\text { service }\end{array}$ & $\begin{array}{l}\text { Respiratory } \\
\text { syndrome, } \\
\text { cardiovascular } \\
\text { syndrome }\end{array}$ & 14 & $\mathrm{Y}$ & $\mathrm{R}$ & $(41)$ \\
\hline Spain & $\begin{array}{l}\text { SIDARTHa- } \\
\text { Cantabria }\end{array}$ & $\begin{array}{l}\text { Emergency } \\
\text { department }\end{array}$ & $\begin{array}{l}\text { No syndrome } \\
\text { specific analysis } \\
\text { (only total number } \\
\text { of cases) }\end{array}$ & 14 & $\mathrm{Y}$ & $\mathrm{R}$ & $(41)$ \\
\hline Sweden & GET WELL & Web queries & Not known & $\begin{array}{l}\text { Not } \\
\text { known }\end{array}$ & $\mathrm{N}$ & Not known & $(29,31)$ \\
\hline $\begin{array}{l}\text { United } \\
\text { Kingdom } \\
\text { (England) }\end{array}$ & $\begin{array}{l}\text { Q-Surveillance } \\
\text { (scheduled care } \\
\text { surveillance } \\
\text { England) }\end{array}$ & Primary care & $\begin{array}{l}\text { Asthma, } \\
\text { conjunctivitis, } \\
\text { allergic rhinitis, } \\
\text { wheeze, } \\
\text { lower respiratory } \\
\text { tract infection, } \\
\text { upper respiratory } \\
\text { tract infection }\end{array}$ & 1 & $\mathrm{~N}$ & $\mathrm{P}$ & $(42)$ \\
\hline $\begin{array}{l}\text { United } \\
\text { Kingdom } \\
\text { (Scotland) }\end{array}$ & $\begin{array}{l}\text { NHSDirect } \\
\text { surveillance } \\
\text { England/Wales }\end{array}$ & $\begin{array}{l}\text { Telephone } \\
\text { helpline }\end{array}$ & $\begin{array}{l}\text { Difficulty } \\
\text { breathing, } \\
\text { eye problems, } \\
\text { cough, rash }\end{array}$ & 2 & $\mathrm{Y}$ & $P$ & $(42)$ \\
\hline
\end{tabular}

* Europe = EU Member States, European Free Trade Zone countries, Acceding and Candidate countries

+ First report after first day of volcanic eruption

\section{Added value of syndromic surveillance at the subnational level during three public health emergencies}

During the influenza pandemic, 13 out of 15 systems provided syndromic information at the subnational level, while nine systems and the European study provided data at the national level (table 1). Smith et al. (32), Kavanagh et al. (37), and Todd et al. (36) highlighted the use of local analyses for earlier identification of the onset of the pandemic in certain regions compared to the national level analysis. Rosenkötter et al. compared among others ambulance patient records at the local level in one country with national level data in another and found better validity for national level data (21). The low validity at the local level was explained by the difficulty to differentiate signal from noise when very small case numbers were analysed. The authors also compared single emergency departments located at the subnational level in two different countries and found that both provided syndromic data of sufficient validity and timeliness compared to sentinel data in the regions. Case numbers at these emergency departments were large enough for a sound syndromic surveillance analysis. These examples show that a valid application at the subnational level is achievable if the analysed data source can potentially reflect a critical mass of cases. 
During the volcanic ash plume event, information in Sweden (29) and the United Kingdom (UK) was only published for the national level, i.e., England and Scotland, however, in Scotland also the subnational level was analysed but no results were presented (42). The systems in Austria, Germany and Spain were providing information at the subnational level (41). As during the influenza pandemic, the systems in Austria and Germany were analysing low case numbers which was affecting the validity of the results.

During the 0104:H4 outbreak, 193 German emergency departments participated in total, of which 28 were located in the more effected areas. The subnational syndromic data were aggregated to county level and were assessed as suitable for the timely analysis of the development of the outbreak (44).

In conclusion, syndromic surveillance can support public health authorities at the subnational level to detect events earlier or rapidly gain information about the health impact of an event. Although many syndromic surveillance systems are applied at the subnational level, the added value compared to a national application is often not analysed. The problem of distinguishing signals from noise, especially of events with low case numbers, also became apparent during other syndromic surveillance applications at subnational or national level $(47,48)$.

\section{All-hazard applicability of syndromic surveillance}

The examples showed that syndromic surveillance was used to assess the health impact of different (potential) communicable and non-communicable PHEIC which is in line with the all-hazard requirement of the IHR (2005). Although these are only three examples, the flexibility of syndromic surveillance to analyse different kinds of health threats was shown during many subnational or national level applications in Europe, such as massgatherings, e.g., the Olympic Games in the UK, environmental events, e.g., heat and cold waves or floods, and diverse communicable disease outbreaks $(13,20)$. Also Paterson and colleagues highlighted the 'remarkable adaptability of syndromic surveillance' in their recent review (15).

\section{Comparability of syndromic surveillance output for cross-border surveillance in Europe}

We explore the European comparability of syndromic surveillance systems, especially in terms of compliance to European case definitions used during the $\mathrm{A} / \mathrm{H} 1 \mathrm{~N} 1$ influenza pandemic and the O104: $\mathrm{H} 4$ outbreak (6).

During the influenza pandemic, the 15 systems and the European study analysed influenza-like illness (ILI) or respiratory symptoms based on different case definitions (table 1). Some systems applied the Centers for Disease Control and Prevention (CDC) definition for ILI $(26,49)$ or the European ILI definition established by $\operatorname{ECDC}(28)$ which are based on symptoms. Others use aggregated diagnostic information and not symptoms, self-defined queries of standardised coding categories or free-text. Also for syndromic surveillance systems based on the same group of data sources, for instance emergency departments, the diagnostic coding systems differ. It might not be achievable to use the European definition in a syndromic surveillance system because the underlying source does not provide all necessary information. For example, the use of the European definition was attempted for the syndromic surveillance system in Sweden but the 
analysed data source of web queries did not provide all necessary information (29). For the system in Ireland, different definition for free-text searches were compared, one being based on the national definition, another one on the CDC definition, but the best outcome for identifying influenza cases was achieved using a definition which was tailored to the available information in the data source (25). Other syndromic surveillance systems do not use a definition but analyse the total volume which is reducing specificity, for example systems using school absenteeism data $(35,50,51)$. These examples show that not one and the same case definition can and should be applied when using syndromic surveillance in order to achieve valid results. Although this means that these case definitions are not comparable, most systems analysed similar syndromes indicating ILI.

None of the examples provided evidence that syndromic surveillance is capable of identifying an A/H1N1 case according to the full European clinical case definition. The syndromic surveillance systems could help identify cases meeting a part of the clinical case definition, always depending on the information provided by the analysed data source.

The EU did not provide any case definition during the volcanic ash plume event. The cross-border comparability of the definitions used by the different syndromic surveillance systems is to be considered weak due to differences in the analysed data sources as seen also during the A/H1N1 pandemic. During the O104:H4 outbreak, the syndromic surveillance output could not meet the European clinical case definition of Shiga ToxinProducing Escherichia coli (STEC)-associated Haemolytic Uremic Syndrome (HUS), only for STEC/Verotoxin producing Escherichia coli related diarrhoea. Therefore, syndromic surveillance could not contribute to clinical STEC-associated HUS case classification. However, the European comparability of the syndrome "bloody diarrhoea" can be considered high as it is based on a relatively simply defined symptom which is clearly distinguishable from other symptoms.

To conclude, syndromic surveillance can contribute to identifying clinical cases according to the European case definitions but it is unlikely that syndromic information provides more detailed information to identify cases according to full clinical case definitions as required by the IHR (2005). Syndromic surveillance is rather suited to augment existing surveillance systems in order to provide a timely indication of clinical cases to be verified by traditional surveillance and assessment measures.

The low level of comparability of syndromic information in Europe in terms of case definitions, data formats and diagnostic coding does not speak for implementing standardised syndromic surveillance systems at European level or for aiming at a harmonisation of existing systems in order to support cross-border surveillance. From a pragmatic point of view one could argue that the systems are analysing similarly defined syndromes, such as ILI, and are in this way enabling cross-border surveillance. Also the European influenza surveillance system is based on different data sources and different case definitions of ILI or acute respiratory illness (52-54).

Following this line of argument, the Triple S-AGE project suggested three models of syndromic surveillance harmonization in Europe: (A) a European syndromic surveillance system model with standardised data sources and case definitions as implemented for mortality monitoring in the European Mortality Monitoring system (55), (B) a completely disharmonised European model as it is existing at the moment for all morbidity syndromic surveillance systems, and (C) an intermediate model with diverse data sources and case 
definitions but standardised reporting at the European level (56). Depending on the characteristics of the syndrome/event and the opportunity to generate comparable syndromes from the available data, these models can exist in parallel. From a European point of view, the goal might be to find the optimal combination of these models with the largest possible harmonisation to allow for cross-border comparison. In the following section, we will look at the strengths and weaknesses of different implementation models of syndromic surveillance at the subnational level in Europe (following the Triple S-AGE models $B$ and $C$ ).

\section{Strengths and weaknesses of implementation models for subnational syndromic surveillance in Europe}

Current subnational syndromic surveillance implementation: national vs. local model

The implementation of syndromic surveillance systems for subnational surveillance in European countries currently follows either a national or a local model (table 1). Syndromic surveillance systems following the national model are hosted at the national level, collecting subnational data, collating and analysing it at the national level, and reporting surveillance results at the national and subnational level. Examples for national syndromic surveillance systems in Europe are especially found in the UK $(32,34,37,50$, $57)$ and France $(58,59)$, which are the longest established syndromic surveillance systems in Europe. This might be explained by the fact that both countries have centrally organised health systems (20). Implementation of national systems is likely to be limited in federal countries with a long tradition of predominantly decentralised health systems (10).

Syndromic surveillance systems following the local model are hosted at the local level, collating and analysing data covering a single jurisdiction and reporting surveillance results for that jurisdiction only. Examples for local syndromic surveillance systems are found in many countries (table 1 ), also in countries having national syndromic surveillance systems, e.g., in Italy $(26,60)$, and the UK $(35,61)$.

Table 4 compares the strengths and weaknesses of the two models for some syndromic surveillance system characteristics for which we could identify an impact by the model of implementation. The characteristics are defined following the framework for assessing syndromic surveillance systems proposed by the CDC (62) and detailed by the Triple SAGE project (63).

National systems have the biggest advantage in simplicity and cost-effectiveness. To set up a syndromic surveillance system at the national level without involving too many stakeholders, based on national registries or a national point of access to a network of data providers is relatively easy. Examples for such systems are found in Belgium (21), France (64) or the UK (36). If data collection is organised regionally or different data collection software is used as it is often the case for health care services, access to data sources can be difficult, impeding representativeness of the whole country and delaying setup of a system, as reported for example for syndromic surveillance systems in England (20).

Comparability of surveillance system results in national systems is high as data source, case definitions, analysis methodology, and reporting are the same for the whole country. The chance of detecting events covering multiple subnational jurisdictions is high in a 
national system and impossible in a local system. The signal-to-noise problem of syndromic surveillance which limits detection of events with small case numbers is the same for both models.

A single national or local syndromic surveillance system may be prone to become a 'single point of failure' in times of crises, because of a power failure, for example (7). A decentralised surveillance system can provide information during an event even if parts of the system are not working.

The biggest advantage of the local syndromic surveillance model is that it can be adjusted to the local circumstances of data availability and accessibility, data collection procedures, treatment seeking behaviour of the population and priorities for targeted health threats. Syndromic surveillance performs best if fine-tuned to the characteristics of the analysed data source as shown for the influenza syndromic surveillance system in Spain, for example (28).

A local system which is operated by the professionals responsible for local surveillance is more easily "owned" by these professionals and will foster motivation and quality of work, compared to a system which is imposed by the national level $(20,65)$.

A close collaboration between local public health authorities and local health care institutions which often function as data providers can simplify the setup and maintenance of syndromic surveillance systems. Data providers know their data and the context of data collection best. They can identify the most suitable data fields for analysis which improves data quality, and they can support interpretation of syndromic surveillance results by giving explanations for signals. Collaboration between health care institutions and public health authorities for a syndromic surveillance system can enhance collaboration of these two often divided parts of the health system for crisis preparedness (10). Finally, syndromic surveillance output based on health care data can also be useful to support clinical resource management in health care institutions during times of crisis (8).

Another advantage of local systems is that raw data are analysed in the same local jurisdiction compared to a national system where data are leaving the jurisdiction to be analysed at the national level. This can reduce problems of data privacy that are arising from analysing patient information (66).

In summary, there are distinct advantages and disadvantages of the national and the local syndromic surveillance system models which are hampering to make the best use of syndromic surveillance. Systems should at best be set up locally adjusted but also covering a large part of the population. In the following we propose a system model that combines these advantages of the national and local system model. 
We suggest a new implementation model in which syndromic surveillance is integrated in existing surveillance structures at the subnational level. Data collation and analysis are done at the local level, while higher levels can analyse aggregated syndromic data or only receive reports. This is to be decided in each country and will depend on the organisation of the public health and surveillance system. Transferring aggregated instead of raw data or reports to higher levels has the advantage that data privacy rules are respected. Reporting of surveillance results for the local, regional and national level is following a standardised format. The approach can only live up to its potential by covering a large part of the population and several jurisdictions.

In the EU project "European Emergency Data Based System for Information on, Detection and Analysis of Risks and Threats to Health" (SIDARTHa), we developed a subnational syndromic surveillance implementation model based on three different emergency care data sources (67). Figure 1 depicts the setup, data flow and reporting directions in a SIDARTHa syndromic surveillance system. A decision tree was developed for the SIDARTHa approach for validation of signals so that not every syndromic signal results in a public health response (68). Currently, the SIDARTHa system is implemented in one region in the countries of Austria (active), Belgium (pilot), Germany (pilot) and Spain (active) $(21,68)$.

Table 4 compares the strengths and weaknesses of the subnational model compared to the local and national model. The model combines the advantages of the local and the national model for most characteristics. An additional improvement of this model is the stability in times of crisis by the decentralised setup of systems. Further, representativeness is anticipated to be increased through easier access to subnational data sources by subnational surveillance system operators. However, comparability of surveillance results in terms of case definitions, data formats and diagnostic coding systems can be anticipated lower as in a national system setup. Systems following the subnational model are likely to differ in their setup because they are adjusted to the local accessibility and characteristics of data sources in each region. The comparability can be increased if a common framework is used for setting up a system, as for example developed by the European projects SIDARTHa and Triple S-AGE.

The major disadvantage in comparison to the local and national model is the higher effort and complexity in the setup of a decentralised and locally adjusted system. Because of the high effort, the two active SIDARTHa systems in Austria and Spain could only be implemented as local systems providing information for one region in the respective country. For a roll-out to other regions we consider political endorsement and support from higher levels as vital. 
Table 4: Strengths and weaknesses of syndromic surveillance system implementation models in Europe*

\begin{tabular}{|c|c|c|c|}
\hline Syndromic surveillance system characteristics & $\begin{array}{l}\text { National } \\
\text { model }^{+}\end{array}$ & $\begin{array}{l}\text { Local } \\
\text { model }^{\ddagger}\end{array}$ & $\begin{array}{l}\text { Integrated } \\
\text { subnational } \\
\text { model }^{\S}\end{array}$ \\
\hline Simplicity and costs of setup & ++ & + & -- \\
\hline Simplicity of access to subnational data sources & - & + & + \\
\hline Stability: Potential of single system failure in times of crisis & -- & -- & ++ \\
\hline Acceptance and utilization of syndromic surveillance results at subnational level & - & ++ & ++ \\
\hline Flexibility of adjustment to local events/priorities & - & ++ & ++ \\
\hline Data protection problems & - & ++ & ++ \\
\hline Data quality & - & ++ & ++ \\
\hline Validity: Interpretation of signals including false alerts (signal-to-noise problem) & - & + & + \\
\hline Validity: Small-number problem in detecting local events & -- & -- & -- \\
\hline Validity: Detection of events covering multiple local jurisdictions & + & -- & + \\
\hline Representativeness of whole country & + & -- & ++ \\
\hline Comparability of surveillance results across multiple subnational jurisdictions & ++ & -- & + \\
\hline Transferability between subnational jurisdictions & + & -- & + \\
\hline Clinical resource and quality management in health care institutions & -- & + & + \\
\hline Crisis preparedness of health care institutions & -- & + & + \\
\hline
\end{tabular}

* Europe = EU Member States, European Free Trade Zone countries, Acceding and Candidate countries

+ Data collation and analysis at national level, representing several subnational jurisdictions, top-down reporting to national, regional and local level

\# Data collation and analysis at local level, representing a single subnational jurisdiction, local reporting to local level

$\S$ Data collation and analysis at local level, analysis of aggregated data at regional or national level representing several subnational jurisdictions, standardised bottom-up reporting to local, regional and national level

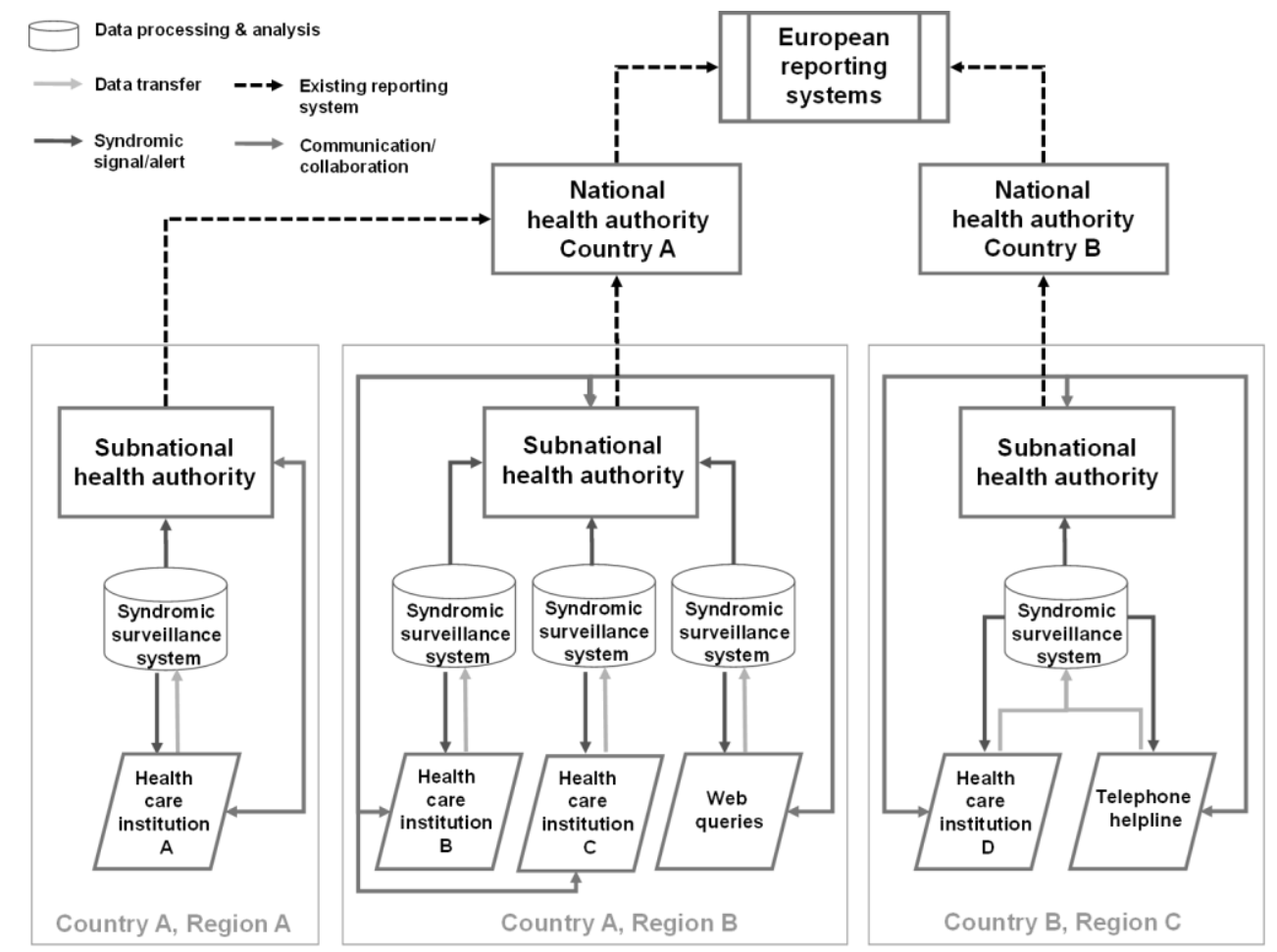

Figure 1: The SIDARTHa model for integrated syndromic surveillance at the subnational level

SIDARTHa syndromic surveillance systems are implemented at subnational level and can be based on one or different kinds of data sources. In this way, the data analysis algorithms can be chosen and adjusted according to the immediate context. The syndromic surveillance results feed into the established surveillance and reporting system of the responsible subnational health authority augmenting existing (traditional) surveillance information. Syndromic information would only be reported to higher levels in aggregated form limiting problems arising from data privacy. Investigation of signals is done at subnational level but could also be done at national levels to allow for detection of events covering several jurisdictions. The data providing institutions should also receive access to syndromic surveillance results for their institution and/or jurisdiction which could be used by them for resource planning purposes. 


\section{Summary}

We conclude that syndromic surveillance can support countries to detect and assess the public health impact of different types of PHEIC at the subnational level as stipulated in the IHR (2005) core surveillance capacity requirements. The approach provided timely information during three different public health emergencies in Europe. For some events syndromic surveillance systems were the only available source of near real-time information. Many syndromic surveillance systems are applied at the subnational level, which in some cases proved to be of advantage for detecting an event earlier compared to the national level. Syndromic surveillance is not suited to detect local events consisting of small case numbers.

In terms of case definitions, data formats or diagnostic coding systems, syndromic surveillance systems are not identical across Europe because of the diversity of the analysed data sources. Nevertheless, we consider comparable cross-border surveillance possible based on similarly defined syndromes.

Implementation of syndromic surveillance in Europe currently follows either a local or national model. In order to gain the most of syndromic surveillance we suggest a new subnational approach of implementing syndromic surveillance. The model foresees locally adjusted data collation and analysis at the subnational level, and integrated and standardised reporting to higher levels. By covering a large part of the population, events covering several jurisdictions can be identified at higher levels. However, the setup of decentralised and locally adjusted systems is more complex compared to setup of one national or local system.

Using guidelines and tools produced by European syndromic surveillance projects and with national or European policy support, a wider roll-out of syndromic surveillance across Europe can be achieved. Only by expanding the application of syndromic surveillance, European countries will be positioned to timely assess the public health impact of potential PHEIC, especially rare and non-communicable events.

\section{Endnotes}

a The literature search was accomplished in June 2013 and updated in July 2014. The search string for PubMed was: (H1N1(Title/Abstract) OR pandemic(Title/Abstract)) AND "syndromic surveillance"(Title/Abstract). The search string for Google Scholar was: H1N1 OR pandemic AND "syndromic surveillance". The review of Google Scholar hits stopped after 10 pages of hits which did not provide any new relevant content. We checked references of selected full-text articles for further relevant publications.

b The additional identified systems were the SurSauUD emergency department and general practitioner house calls surveillance systems in France, the national emergency department surveillance system and the Lazio emergency department surveillance system in Italy, the South Holland South general practitioner pandemic surveillance system in the Netherlands, the PIPeR general practitioner pandemic surveillance system in Scotland, the general practitioner out-of-hours surveillance system in Ireland, and the general practitioner surveillance system in Wales. 


\section{Acknowledgements}

This paper arises from the project SIDARTHa which has received funding from the European Union, in the framework of the Public Health Programme (grant agreement number 2007208).

The authors would like to thank the three reviewers for their valuable comments on earlier versions of this article. 


\section{References}

1. World Health Organization. International Health Regulations (2005). Geneva: World Health Organization; 2008.

2. Paquet C, Coulombier D, Kaiser R, Ciotti M. Epidemic intelligence: a new framework for strengthening disease surveillance in Europe. Euro Surveill. $2006 ; 11(12): 212-214$.

3. World Health Organization. European Strategy Meeting for implementation of the International Health Regulations (2005) - Scope and purpose. Copenhagen: World Health Organization; 2013.

4. Allebeck P. Which health data for Europe? Eur J Public Health. 2012;22(5):611.

5. European Parliament and Council of the European Union. Decision on serious cross-border threats to health and repealing Decision No 2119/98/EC (1082/2013/EU). Off J Eur Union. 2013;L 293:1-15.

6. European Commission. Commission Implementing Decision amending Decision 2002/253/EC laying down case definitions for reporting communicable diseases to the Community network under Decision No 2119/98/EC of the European Parliament and of the Council (2012/506/EU). Off J Eur Union. 2012;L262:1-57.

7. Mostashari F, Hartman J. Syndromic surveillance: a local perspective. J Urban Health. 2003;80(2 Suppl 1):i1-i7.

8. McManus J, Huebner K, Scheulen J. The science of surge: Detection and situational awareness. Acad Emerg Med. 2006;13(11):1179-82.

9. Lyytikainen O, Kuusi M, Snellman M, Virtanen M, Eskola J, Ronkko E, et al. Surveillance of influenza in Finland during the 2009 pandemic, 10 May 2009 to 8 March 2010. Euro Surveill. 2011;16(27). pii: 19908.

10. Wilson K, McDougall C, Forster A. The responsibility of healthcare institutions to protect global health security. Healthc Q. 2009;12(1):56-60.

11. Altmann M, Spode A, Altmann D, Wadl M, Benzler J, Eckmanns T, et al. Timeliness of surveillance during outbreak of Shiga Toxin-producing Escherichia coli infection, Germany, 2011. Emerg Infect Dis. 2011;17(10):1906-9.

12. Triple S. Assessment of syndromic surveillance in Europe. Lancet. 2011;378 (9806): 1833-1834.

13. Conti S, Kanieff M, Rago $G$, on behalf of the Triple-S project. Inventory of Syndromic Surveillance Systems in Europe; 2012. Available from: http://www.syndromicsurveillance.eu/images/stories/Final_material/tripleS_country_visits.pdf.

14. Ziemann A, Krafft T. Guidelines for Assessment of Data Sources; 2013. Available from: http://www.syndromicsurveillance.eu/triple-s_guidelines_datasources. pdf.

15. Paterson BJ, Durrheim DN. The remarkable adaptability of syndromic surveillance to meet public health needs. J Epidemiol Global Health. 2013;3(1):41-7.

16. Kaydos-Daniels SC, Rojas Smith L, Farris TR. Biosurveillance in outbreak investigations. Biosecur Bioterror. 2013;11(1):20-8. 
17. Koopmans M. Surveillance strategy for early detection of unusual infectious disease events. Curr Opin Virol. 2013;3(2):185-91.

18. Morse SS. Public health surveillance and infectious disease detection. Biosecur Bioterror. 2012;10(1):6-16.

19. Revision of the International Health Regulations. Progress report, February 2001. Wkly Epidemiol Rec. 2001;8:61-3.

20. Ziemann A, Krafft T, Sala Soler M, Sypniewska P. Country visits. Triple S-AGE project; 2013. Available from: http://syndromicsurveillance.eu/images/ stories/Final_material/triple-S_country_visits.pdf

21. Rosenkötter N, Ziemann A, Garcia-Castrillo Riesgo L, Gillet JB, Vergeiner G, Krafft $T$, et al. Validity and timeliness of syndromic influenza surveillance during the autumn/winter wave of $A(H 1 N 1)$ influenza 2009: results of emergency medical dispatch, ambulance and emergency department data from three European regions. BMC Public Health. 2013;13(1):905. doi: 10.1186/1471-2458-13-905.

22. Bollaerts K, Antoine J, Robesyn E, Van Proeyen L, Vomberg J, Feys E, et al. Timeliness of syndromic influenza surveillance through work and school absenteeism. Arch Public Health. 2010;68:115-20.

23. Harder KM, Andersen PH, Baehr I, Nielsen LP, Ethelberg S, Glismann S, et al. Electronic real-time surveillance for influenza-like illness: experience from the 2009 influenza $A(H 1 N 1)$ pandemic in Denmark. Euro Surveill. 2011;16(3). pii: 19767

24. Gerbier-Colomban S, Potinet-Pagliaroli V, Metzger MH. Can epidemic detection systems at the hospital level complement regional surveillancenetworks: case study with the influenza epidemic? BMC Infect Dis. 2014;14:381.

25. Brabazon ED, Carton MW, Murray C, Hederman L, Bedford D. General practice out-of-hours service in Ireland provides a new source of syndromic surveillance data on influenza. Euro Surveill. 2010;15(31). pii: 19632.

26. Ansaldi F, Orsi A, Altomonte F, Bertone G, Parodi V, Carloni R, et al. Emergency department syndromic surveillance syndromic surveillancetem for early detection of 5 syndromes: a pilot project in a reference teaching hospital in Genoa. Italy J Prev Med Hyg. 2008;49(4):131-5.

27. Amicizia D, Cremonesi I, Carloni R, Schiaffino S. The response of the Liguria Region (Italy) to the pandemic influenza virus A/H1N1sv. J Prev Med Hyg. $2011 ; 52(3): 120-3$.

28. Schrell S, Ziemann A, Garcia-Castrillo Riesgo L, Rosenkötter N, Llorca J, Popa D, et al. Local implementation of a syndromic influenza surveillance syndromic surveillancetem using emergency department data in Santander, Spain. J Public Health (Oxf). 2013;35(3):397-403.

29. Hulth A, Rydevik G, Linde A. Web queries as a source for syndromic surveillance. PLoS One. 2009;4(2):e4378. doi: 10.1371/journal.pone.0004378.

30. Hulth $A$, Rydevik G. Web query-based surveillance in Sweden during the influenza A(H1N1)2009 pandemic, April 2009 to February 2010. Euro Surveill. 2011;16(18). pii: 19856. 
31. Hulth A, Rydevik G. GET WELL: an automated surveillance syndromic surveillancetem for gaining new epidemiological knowledge. BMC Public Health. 2011;11:252. doi: 10.1186/1471-2458-11-252.

32. Smith S, Smith GE, Olowokure B, Ibbotson S, Foord D, Maguire H, et al. Early spread of the 2009 influenza $A(H 1 N 1)$ pandemic in the United Kingdom-use of local syndromic data, May-August 2009. Euro Surveill. 2011;16(3). pii: 19771.

33. Cooper DL, Verlander NQ, Elliot AJ, Joseph CA, Smith GE. Can syndromic thresholds provide early warning of national influenza outbreaks? J Public Health (Oxf). 2009;31(1):17-25.

34. Harcourt SE, Smith GE, Elliot AJ, Pebody R, Charlett A, Ibbotson S, et al. Use of a large general practice syndromic surveillance syndromic surveillance system to monitor the progress of the influenza $\mathrm{A}(\mathrm{H} 1 \mathrm{~N} 1)$ pandemic 2009 in the UK. Epidemiol Infect. 2012;140(1):100-5.

35. Kara EO, Elliot AJ, Bagnall H, Foord DG, Pnaiser R, Osman $H$, et al. Absenteeism in schools during the 2009 influenza $A(H 1 N 1)$ pandemic: a useful tool for early detection of influenza activity in the community? Epidemiol Infect. 2012;140(7):1328-36.

36. Todd S, Diggle PJ, White PJ, Fearne A, Read JM. The spatiotemporal association of non-prescription retail sales with cases during the 2009 influenza pandemic in Great Britain. BMJ Open. 2014;4(4):e004869. doi: 10.1136/bmjopen-2014004869.

37. Kavanagh K, Robertson C, Murdoch H, Crooks G, McMenamin J. Syndromic surveillance of influenza-like illness in Scotland during the influenza A H1N1V pandemic and beyond. J R Stat Soc (Series A). 2012;175:939-58.

38. Valdivia A, Lopez-Alcalde J, Vicente M, Pichiule M, Ruiz M, Ordobas M. Monitoring influenza a ctivity in Europe with Google Flu Trends: comparison with the findings of sentinel physician networks - results for 2009-10. Euro Surveill. 2010;15(29). pii: 19621.

39. European Centre for Disease Prevention and Control. Second Interim Threat Assessment. Ash cloud following volcanic eruption in Iceland, 20 April 11:00 CET. Stockholm: European Centre for Disease Prevention and Control; 2010.

40. World Health Organization Regional Office for Europe. WHO/Europe expert group concludes Icelandic volcanic ash currently poses no threat to public health [Internet]. Available from: http://www.euro.who.int/en/healthtopics/environment-and-health/air-quality/news/news/2010/05/whoeuropeexpert-group-concludesicelandic-volcanic-ash-currently-poses-no-threat-topublic-health.

41. Rosenkötter N, Ziemann A, Garcia-Castrillo Riesgo L, Vergeiner G, Fischer M, Krafft $T$, et al. SIDARTHa Volcanic Ash Cloud Rapid Public Health Impact Assessment. Regional public health impact of volcanic ash cloud covering Europe after eruption of Eyjafjallajoekull, Iceland starting April 14th, 2010. Results as of May 15th, 2010. Bad Honnef: SIDARTHa project; 2010. Available from: http://ec.europa.eu/chafea/documents/health/SIDARTHa_rapid_assessment_vo Icanic_ash_cloud_15MAY2010.pdf. 
42. Elliot AJ, Singh N, Loveridge $P$, Harcourt S, Smith S, Pnaiser R, et al. Syndromic surveillance to assess the potential public health impact of the Icelandic volcanic ash plume across the United Kingdom, April 2010. Euro Surveill. 2010;15(23). pii: 19583.

43. Robert Koch Institut. Final presentation and evaluation of epidemiological findings in the EHEC O104:H4 Outbreak, Germany 2011. Berlin: Robert KochInstitute; 2011.

44. Wadl M, Rieck T, Nachtnebel M, Greutelaers B, an der Heiden M, Altmann D, et al. Enhanced surveillance during a large outbreak of bloody diarrhoea and haemolytic uraemic syndrome caused by Shiga toxin/verotoxinproducing Escherichia coli in Germany, May to June 2011. Euro Surveill. 2011;16(24). pii: 19893.

45. Dailey L, Watkins RE, Plant AJ. Timeliness of data sources used for influenza surveillance. J Am Med Inform Assoc. 2007;14(5):626-31.

46. Rosenkötter N, Ziemann A, Krafft T, Riesgo LG, Vergeiner G, Brand H. Noninfectious events under the International Health Regulations (2005) in Europe a case for syndromic surveillance. J Public Health Policy. 2014;35(3):311-26.

47. Coory MD, Kelly H, Tippett V. Assessment of ambulance dispatch data for surveillance of influenza-like illness in Melbourne, Australia. Public Health. 2009;123(2):163-8.

48. Xing J, Burkom $\mathrm{H}$, Tokars J. Method selection and adaptation for distributed monitoring of infectious diseases for syndromic surveillance. J Biomed Inform. 2011;44(6): 1093-101.

49. van den Wijngaard $C$, van Asten $L$, van Pelt W, Nagelkerke NJ, Verheij $R$, de Neeling $A J$, et al. Validation of syndromic surveillance for respiratory pathogen activity. Emerg Infect Dis. 2008;14(6):917-25.

50. Mook P, Joseph C, Gates P, Phin N. Pilot scheme for monitoring sickness absence in schools during the 2006/07 winter in England: can these data be used as a proxy for influenza activity? Euro Surveill. 2007;12(12):E11-E12.

51. Schmidt WP, Pebody R, Mangtani P. School absence data for influenza surveillance: a pilot study in the United Kingdom. Euro Surveill. 2010;15(3). pii: 19467.

52. Aguilera JF, Paget WJ, Mosnier A, Heijnen ML, Uphoff $H$, van der Velden J, et al. Heterogeneous case definitions used for the surveillance of influenza in Europe. Eur J Epidemiol. 2003;18(8):751-4.

53. Paget J, Marquet R, Meijer A, van der Velden K. Influenza activity in Europe during eight seasons (1999-2007): an evaluation of the indicators used to measure activity and an assessment of the timing, length and course of peak activity (spread) across Europe. BMC Infect Dis. 2007;7:141. Doi: 10.1186/1471-2334-7-141.

54. Kissling E, Valenciano $M$, Falcao J, Larrauri A, Widgren $K$, Pitigoi D, et al. "IMOVE" towards monitoring seasonal and pandemic influenza vaccine effectiveness: lessons learnt from a pilot multi-centric case-control study in Europe, 2008-9. Euro Surveill. 2009;14(44). pii: 19388. 
55. EuroMOMO [Internet]. Available from: http://www.euromomo.eu.

56. Medina S, Fouillet A, Ziemann A, Krafft T, Cooper D, Dupuy C, et al. Proposal for a European strategy for syndromic surveillance. Toward comparability of reporting from syndromic surveillance systems in Europe; 2013. Available from: http://syndromicsurveillance.eu/Triple-S_proposal.pdf.

57. Cooper DL, Smith G, Baker M, Chinemana F, Verlander N, Gerard E, et al. National symptom surveillance using calls to a telephone health advice service-United Kingdom, December 2001-February 2003. MMWR Morb Mortal Wkly Rep. 2004;53(Suppl):179-83.

58. Flamand C, Larrieu S, Couvy F, Jouves B, Josseran L, Filleul L. Validation of a syndromic surveillance syndromic surveillancetem using a general practitioner house calls network, Bordeaux, France. Euro Surveill. 2008;13(25). pii: 18905.

59. Josseran L, Nicolau J, Caillere N, Astagneau P, Brucker G. Syndromic surveillance based on emergency department activity and crude mortality: two examples. Euro Surveill. 2006;11(12):225-9.

60. Guasticchi G, Giorgi Rossi P, Lori G, Genio S, Biagetti F, Gabriele S, et al. Syndromic surveillance: sensitivity and positive predictive value of the case definitions. Epidemiol Infect. 2009;137(5):662-71.

61. Davies GR, Finch RG. Sales of over-the-counter remedies as an early warning syndromic surveillancetem for winter bed crises. Clin Microbiol Infect. 2003;9(8):858-63.

62. Buehler JW, Hopkins RS, Overhage JM, Sosin DM, Tong V. Framework for evaluating public health surveillance systems for early detection of outbreaks: recommendations from the CDC Working Group. MMWR Recomm Rep. 2004;53(RR-5):1-11.

63. Ziemann A, Krafft T. Scientific visit guidelines for knowledge exchange on syndromic surveillance in Europe. Triple S-AGE project; 2011. Available from: http://syndromicsurveillance.eu/triple-s_scientific_visit_guidelines.pdf.

64. Bounoure F, Beaudeau P, Mouly D, Skiba M, Lahiani-Skiba M. Syndromic surveillance of acute gastroenteritis based on drug consumption. Epidemiol Infect. $2011 ; 139(9): 1388-95$.

65. Buehler JW, Whitney EA, Smith D, Prietula MJ, Stanton SH, Isakov AP. Situational uses of syndromic surveillance. Biosecur Bioterror. 2009;7(2):165-77.

66. EuroREACH. Good practice on data linkages and performance measurement in relation to access to national health care data systems. Available from: http://www.euroreach.net/sites/default/files/EuroREACHWP3\%20Final\%20Report.pdf.

67. SIDARTHa [Internet]. Available from: http://www.sidartha.eu.

68. Ziemann A, Rosenkötter N, Garcia-Castrillo Riesgo L, Schrell S, Kauhl B, Vergeiner $G$, et al. A concept for routine emergency-care data-based syndromic surveillance in Europe. Epidemiol Infect. 2014;142(11):2433-46. 



\section{CHAPTER 6}

\section{Success Factors of European Syndromic Surveillance Systems}

submitted as:

Ziemann A, Fouillet A, Brand H, Krafft T. Success factors of European syndromic surveillance systems: A Qualitative Comparative Analysis. 


\section{Abstract}

Syndromic surveillance aims at augmenting traditional public health surveillance with timely information, based on the analysis of mainly existing data sources such as web searches or patient records. Despite the setup of many syndromic surveillance systems, there is still much doubt about the added value of the approach. The diverse interactions between performance indicators such as timeliness, and various system characteristics make the assessment of syndromic surveillance systems a complex endeavour. We argue that the comparison of different syndromic surveillance systems through Qualitative Comparative Analysis helps to assess performance and identify key success factors.

We compiled case-based, mixed data on performance and characteristics of 19 syndromic surveillance systems in Europe from scientific and grey literature and from site visits. We identified success factors of syndromic surveillance by applying crisp-set Qualitative Comparative Analysis for the two main areas of syndromic surveillance application: seasonal infectious disease surveillance focussing on influenza and situational awareness during different kinds of potentially health threatening events.

We found that a syndromic surveillance system might provide timelier information on seasonal influenza if it analyses non-clinical data sources. Timely situational awareness during different kinds of events is fostered by an automated syndromic surveillance system capable of analysing multiple syndromes. To our surprise, the analysis of multiple data sources was not identified as key success factor for situational awareness.

We recommend to consider the key success factors when taking decisions on designing syndromic surveillance systems. Qualitative Comparative Analysis was of added value to interpret complex and mixed data on small- $\mathrm{N}$ cases in the area of public health surveillance and provided practically relevant results.

Key words: Europe, public health surveillance, comparative study, Qualitative Comparative Analysis 


\section{Introduction}

Syndromic surveillance aims at augmenting traditional public health surveillance systems by providing (near) real-time information on the public health impact of events. To gain a head start, syndromic surveillance mainly analyses data from existing sources that were originally not collected for surveillance purposes (1). Such data sources can be web query logs, telephone helpline registries, or patient or veterinary records (2). Over the past 15 years, an increasing number of syndromic surveillance systems were set up in Europe. They had the main purposes of timely infectious disease outbreak detection and near real-time situational awareness during events such as mass gatherings or extreme weather events (3). However, there is still a lot of doubt about the added value of the approach (4). A lack of clinical specificity which can lead to false alerts and undetected events is considered the major weakness of syndromic surveillance (5). The major advantages are timeliness, flexibility in using the approach for different kinds of health threats, and cost-effectiveness as no additional data have to be collected $(6,7)$. There are many case reports on the application of syndromic surveillance systems including performance measures. But, there is no general synthesis as to when or why syndromic surveillance systems are performing well or not.

Frameworks for evaluating syndromic surveillance systems propose quantitative performance indicators such as timeliness and validity, and more qualitative indicators such as flexibility and acceptability $(8,9)$. These indicators are affected by different characteristics of a syndromic surveillance system. These can be the analysed data source, the data collection, analysis and reporting process, and the purpose for and context in which the system is set up. The vast number and causal relationships of the different aspects make the assessment of syndromic surveillance systems a complex endeavour. Evaluations to date often focus on single, usually quantifiable, performance indicators (10-13). These evaluations only assess one syndromic surveillance system at a time. There are no comparative analyses of several syndromic surveillance systems. Such comparisons could unveil differences in performance and the impact of certain system characteristics on performance. Decision makers could use this information to design syndromic surveillance systems and improve their added value. We aimed at assessing the performance and identifying success factors of syndromic surveillance systems in Europe by applying Qualitative Comparative Analysis (QCA). Further, we aimed at assessing if QCA has an added value as approach and analysis method in the area of public health surveillance.

\section{Methods}

\section{Qualitative Comparative Analysis}

QCA is an approach and analysis method based on Boolean algebra. It allows for a systematic comparative analysis of small-N and especially case-based data which are not suited for statistical analysis such as regression analysis (14). Ragin gives a good introduction to QCA in a short online presentation (15). The method allows to analyse quantitative and qualitative or mixed data to identify if certain conditions or combinations of conditions (in our case characteristics of syndromic surveillance systems) are part of an outcome set (in our case successful syndromic surveillance systems defined by performance indicators). The core of QCA is the process of Boolean minimisation which aims at reducing the complexity of combinations of conditions to a minimum of necessary 
or sufficient conditions (16). Until now, QCA was mainly applied in the social sciences, especially the political sciences. It is relatively new to the health sciences and was applied in a few studies on identifying success factors of health policies or interventions but not in the area of public health surveillance (17-19).

QCA is an iterative process, characterised by a dialogue between the results of the different steps of the QCA and the researcher's case knowledge and expertise. The data input for the QCA is not fixed a priori but is likely to be adjusted in the process to increase validity of the results. Nevertheless, QCA follows a structured approach of predefined steps: (1) building a data table consisting of outcome indicators and conditions, (2) constructing a "truth table" consisting of configurations (combinations of the conditions and the outcome), (3) Boolean minimisation to reduce the complexity of the configurations to necessary or sufficient configurations known as solution terms, and (4) interpretation of the solution terms (16). Steps 2 and 3 are supported by different free software (20).

There are two main QCA variants, differentiated by the characteristics of the data input: crisp set QCA (cs/QSA) analyses dichotomous data while fuzzy set QCA analyses ordinal or interval data or ratios. The selection of the variant depends on the aim of the study and the data basis. Another key aspect of QCA is the inclusion of configurations without an observed outcome, so called logical remainders (16). Thus, QCA does not only analyse the observed cases but includes all logical possible configurations in the minimisation process to further reduce the complexity of the solution terms. Based on the purpose of this study and the characteristics of the small- $\mathrm{N}$, case-based and mixed data, we found CS/QCA to be the suitable analysis method. In the following, we present our configurations for the QCA steps 1 to 3 .

\section{Data table: conceptual model, data basis, outcome indicators and conditions}

\section{Conceptual model}

There are two main advantages of syndromic surveillance: it provides timelier information and information on events for which no other public health information is available. These virtues are mainly applied for two public health surveillance purposes: (1) timelier information on seasonal infectious disease outbreaks, mainly influenza, and (2) real-time situational awareness during events with potential public health impact such as environmental threats or mass gatherings. The latter also includes reassurance that an event has no public health impact. We performed two cs/QCA to identify success factors of syndromic surveillance systems for these two major areas of application.

\section{Data basis}

The data for this study was collected in the framework of the European project Triple SAGE which aimed at supporting a harmonised setup of syndromic surveillance systems across Europe (21). Based on a literature review and an inventory, 60 European syndromic surveillance systems were identified $(2,22) .36$ systems in eight countries and one European consortium were selected for a site visit based on the extent of syndromic surveillance experience. Active, pilot, planned and expired syndromic surveillance systems were visited during nine site visits between June 2011 and June 2012. Data on the visited syndromic surveillance systems were collected through presentations and 
transcripts of the discussions (23). The 18 syndromic surveillance systems initially selected for this study were chosen based on the status of the system, i.e., if it is or was active and is not only a pilot or planned system, AND on sufficient availability of data for the study, defined as results published in peer-reviewed journals AND coverage during the site visits (table 1). In February 2015, we updated the literature review purposefully on the selected systems searching in Google Scholar and PubMed and by hand-searching references. Case-based data for the QCA were compiled from scientific and grey literature and the site visits. The visits provided additional information for the QCA beyond what was reported in scientific publications. A list of the publications included for the QCA is available on request. In the course of the QCA, we chose to add another case from Germany to increase validity of the QCA. This ad-hoc system was not considered for a site visit as the plan for the site visits was already fixed. All necessary data were collected from scientific and grey literature $(24,25)$.

Table 1: Key characteristics of syndromic surveillance systems selected for QCA

\begin{tabular}{lll} 
Country & Name/description of syndromic surveillance systems & Data source \\
\hline Denmark & DMOS surveillance & Primary care \\
& BioAlarm & Emergency medical dispatch centre \\
\hline England & EDSSS & Emergency department \\
& OOH/Unscheduled care surveillance system & Primary care \\
& QSurveillance & Primary care \\
& NHS Direct & Telephone helpline \\
\hline France & SurSaUD - OSCOUR & Emergency department \\
& SurSaUD - SOS Médecins & Primary care \\
\hline Germany & O104:H4 outbreak surveillance & Emergency department \\
\hline Italy & Migrant influx surveillance & Health services at migrant centres \\
& National emergency department surveillance & Emergency department \\
& Genoa SyS system & Emergency department \\
& Lazio Region SyS system & Emergency department \\
\hline Scotland & NHS24 & Telephone helpline \\
& PiPeR / SISRS & Primary care \\
\hline SIDARTHa* & SIDARTHa-Cantabria & Emergency department \\
& SIDARTHa-Tyrol & Emergency medical dispatch centre \\
\hline Sweden & GETWELL & Web queries \\
& 1177 telephone helpline surveillance & Telephone helpline \\
\hline
\end{tabular}

* SIDARTHa = European syndromic surveillance initiative currently comprising two active systems in Cantabria/Spain and Tyrol/Austria

\section{Outcome indicators}

\section{Syndromic influenza surveillance}

It is anticipated that a syndromic surveillance system can provide timely information if it is based on data sources that are providing data earlier in the course of illness. For example, data from web searches or telephone helplines are considered to provide information on people with mild symptoms before they seek health care. In comparison, traditional surveillance data sources such as sentinel general practitioners or laboratory confirmations are providing information on patients with more severe symptoms. Further, it is anticipated that syndromic surveillance provides timelier information if it is based on pre-diagnostic clinical data such as chief complaints, in comparison to confirmed diagnoses $(2,11,26)$. The data basis was largest for seasonal influenza surveillance, therefore, we chose this case for the QCA. The cut-off for differentiating a successful from an unsuccessful case in the QCA was chosen based on the data reported in the cases and 
based on the following theoretic consideration. As traditional influenza surveillance data are usually available on a weekly basis, a successful syndromic surveillance system should provide data at least the week before. Traditional data sources were sentinel general practitioner reports or laboratory confirmations. We defined the cut-off as syndromic influenza-like-illness signal indicating the onset or peak of the influenza season before the week in which a traditional surveillance data source first indicated the same. We used the average timeliness per system if data were reported for several influenza seasons. The outcome indicator for the QCA (QCA coding: OUTCOME) was coded as 1 for successful syndromic surveillance systems with a timeliness of case detection of less than 0 weeks. The outcome was coded as 0 for unsuccessful systems with a timeliness of case detection of 0 or more weeks. Data were available on syndromic influenza surveillance for nine syndromic surveillance systems in six countries (table 2).

\section{Situational awareness}

We anticipate that successful situational awareness is given if a syndromic surveillance system can be applied to different potential public health threats (applicability) and if it provides rapid information (timeliness of reporting) $(7,27,28)$. We defined the cut-off for a successful outcome for applicability as prospective surveillance during more than one different kind of event. The kind of event could refer to four different event types: environmental threats such as the volcanic ash plume 2010, heat waves, or floods, the A/H1N1 pandemic 2009, mass gatherings such as political summits or sporting events, and industrial accidents. The definition of this outcome indicator and its cut-off were chosen based on the reported applications in the cases. The applicability outcome indicator was combined with an indicator for timeliness. Here, success was defined as reporting of syndromic surveillance results referring to the onset of the health impact of an event or to the frequency of reporting of under 3 days. We used the average timeliness if data were reported for several events. If the two outcome indicators contradicted themselves, we decided to weigh applicability higher than timeliness. Most established syndromic surveillance systems provide timely reports as can be seen from the cases but it is more difficult to apply a system to more than one kind of event. Therefore, the outcome indicator for the QCA (OUTCOME) was coded as 1 for successful syndromic surveillance systems with a timeliness of reporting under 3 days and/or with applicability during two or more different kinds of events. The outcome was coded as 0 for unsuccessful systems with a timeliness of 3 or more days and/or applicability during only one kind of event. Data were available on syndromic surveillance systems in nine countries (table 2). 
Table 2: Raw data for cs/QCA of seasonal syndromic influenza surveillance (a) and syndromic situational awareness (b) (grey shaded areas indicate successful cases)

a)

\begin{tabular}{llllllll} 
Cases / Systems & Country & NONCLIN & ACUTE & SUBNAT & AGE & Timeliness detection [weeks] & OUTCOME \\
\hline 1177 & SE & 1 & 0 & 0 & 0 & -0.9 & 1 \\
GETWELL & SE & 1 & 0 & 0 & 0 & -1.5 & 1 \\
Genoa & IT & 0 & 1 & 1 & 1 & -1.9 & 1 \\
SIDARTHa Cant. & SID & 0 & 1 & 1 & 0 & -0.5 & 1 \\
NHS24 & SC & 1 & 0 & 0 & 0 & -1.0 & 1 \\
NHSDirect & EN & 1 & 0 & 1 & 1 & -0.5 & 1 \\
QSurveillance & EN & 0 & 0 & 1 & 0 & 2.0 & 0 \\
Oscour & FR & 0 & 1 & 0 & 0 & 0,0 & 0 \\
SOS Medecins & FR & 0 & 1 & 1 & 0 & -2.5 & 1 \\
\hline
\end{tabular}

b)

\begin{tabular}{|c|c|c|c|c|c|c|c|c|c|c|}
\hline Cases & Events (Systems) & $\begin{array}{l}\text { MULT- } \\
\text { DATA }\end{array}$ & $\begin{array}{l}\text { MULT- } \\
\text { SYND }\end{array}$ & FREETEXT & AUTOM & EXIST & ELEC & $\begin{array}{l}\text { Appli- } \\
\text { cability } \\
\text { [no. } \\
\text { events] }\end{array}$ & $\begin{array}{l}\text { Time- } \\
\text { liness } \\
\text { reporting } \\
\text { [days] }\end{array}$ & OUTCOME \\
\hline $\mathrm{DE}$ & $\begin{array}{l}\text { O104:H4 (ad-hoc } \\
\text { system) }\end{array}$ & 0 & 0 & 0 & 0 & 0 & 0 & 1 & 2 & 0 \\
\hline DK & Pandemic (DMOS) & 0 & 0 & 0 & 1 & 1 & 1 & 1 & 2 & 0 \\
\hline EN & $\begin{array}{l}\text { Various* (EDSSS, } \\
\text { OOH, Qsurv, } \\
\text { NHSDirect) }\end{array}$ & 1 & 1 & 0 & 1 & 1 & 1 & 4 & 2 & 1 \\
\hline$F R$ & $\begin{array}{l}\text { Various* (Oscour, } \\
\text { SOS Medecins) }\end{array}$ & 1 & 1 & 0 & 1 & 1 & 1 & 4 & 0.63 & 1 \\
\hline IT & $\begin{array}{l}\text { Migrant influx (ad- } \\
\text { hoc system) }\end{array}$ & 0 & 1 & 0 & 0 & 0 & 0 & 1 & 4.41 & 0 \\
\hline SC & $\begin{array}{l}\text { Various* (different, } \\
\text { mainly NHS 24) }\end{array}$ & 1 & 1 & 1 & 1 & 1 & 1 & 3 & 0.91 & 1 \\
\hline SE & $\begin{array}{l}\text { Pandemic, Volcanic } \\
\text { ash plume } \\
\text { (GETWELL) }\end{array}$ & 0 & 1 & 1 & 1 & 1 & 1 & 2 & 2 & 1 \\
\hline SID & $\begin{array}{l}\text { Volcanic ash plume, } \\
\text { pandemic (Tyrol, } \\
\text { Cant.) }\end{array}$ & 1 & 1 & 0 & 0 & 1 & 1 & 1 & 14 & 0 \\
\hline
\end{tabular}

$0=$ absent, $1=$ present, $\mathrm{ACUTE}=$ acute care data sources, $\mathrm{AGE}=$ age-group analysis, AUTOM $=$ automated syndromic surveillance system components, DE = Germany, DK = Denmark, ELEC = electronic data collection, EN = England, EXIST = syndromic surveillance system existed before monitored event, FR = France, FREETEXT $=$ free text analysis, IT $=$ Italy, MULTDATA $=$ multiple data sources, MULTSYND $=$ multiple syndromes, NONCLIN $=$ non-clinical data sources, $\mathrm{SC}=\mathrm{Scotland}$, SE $=$ Sweden, SID = SIDARTHa Cantabria and/or Tyrol, SUBNAT = subnational analysis

\section{Conditions}

\section{Syndromic influenza surveillance}

Timeliness of seasonal influenza case detection is anticipated to be mainly influenced by the analysed data source. Non-clinical data sources (NONCLIN) and information collected prior to confirmed diagnoses from acute care data sources (ACUTE) were expected to be of positive influence on timelier case detection. Non-clinical data sources comprised web searches and telephone helplines while clinical sources referred to primary and acute care data sources. Acute care data sources were referring to emergency departments or outof-ours general practitioner services. After having run a first CS/QCA analysis on these 
two factors, a contradictory combination of conditions occurred. That means that the same combination resulted in a positive and a negative outcome in the observed cases. The most frequently chosen solution to resolve this contradiction is to add conditions (16). We chose the analysis of population subgroups as additional factor. The analysis of different age groups (AGE) can positively influence the timeliness of detecting cases. A similar positive effect is anticipated to come from the analysis of small-scale subnational syndromic surveillance data (SUBNAT) compared to traditional surveillance data referring to a higher administrative level. This could refer to regional syndromic compared to national traditional surveillance results, for example.

\section{Situational awareness}

Factors influencing applicability of systems for various events are flexibility and acceptance of the system. A common experience shared during the site visits was that flexibility is positively influenced by the analysis of multiple data sources (MULTDATA) and free text compared to fixed diagnostic codes (FREETEXT) (23). We added the ability to generate multiple syndromes as another possible success factor (MULTSYND) as a logical deduction from the analysis of multiple data sources. Acceptability of the system is positively affected by the collection of electronic compared to paper-based data (ELEC). Furthermore, the automation of data transfer and possibly also of data analysis and reporting (AUTOM) is expected to have a positive effect. The same applies to the existence of a system before the occurrence of the event compared to systems that are set up ad-hoc during an event (EXIST). These three conditions were also anticipated to positively influence reporting time.

\section{Quality of the data table}

In order to check for the quality of the data table for the minimisation process, we analysed the variety of values across outcome, conditions and cases. Further, we analysed the consistency of the conditions for explaining a positive outcome. A limited variety and consistency can reduce the quality and informational value of the cs/QCA results (16). As a general rule, there should be a mix of cases with a negative and a positive outcome, at least one third of the cases should represent a certain condition value and two conditions should not have the same values across the cases. There are no general rules for defining appropriate levels of consistency, this is depending on the study. However, there is a general agreement about the lower level of consistency of 0.75 (29). To increase quality of the data table, the selection of cases and conditions or the ways in defining the conditions can be reconsidered (16).

\section{Syndromic influenza surveillance}

The condition AGE did not show enough variety across all cases and was excluded from the minimisation process. The consistency levels of the three conditions left for the minimisation process were between 0.43 and 0.57 (table 3 ). There is a limitation to be taken into account when analysing the condition ACUTE. That is that only clinical data sources can also be acute data sources. The consistency level for ACUTE is rising to 0.75 when referring only to clinical instead of all cases. Despite the low consistency levels for 
the conditions NONCLIN and SUBNAT, we included the conditions in the analysis and took account of the consistency in the interpretation of the results.

\section{Situational awareness}

For three conditions MULTSYND, EXIST and ELEC, there was not enough variety over all cases and the conditions ELEC and EXIST were showing the same value pattern over all cases. To resolve the similarity of the conditions ELEC and EXIST, we have decided to exclude the condition ELEC from the QCA. Data are more and more electronically available and, thus, this factor will be of lesser relevance in the future compared to the factor of existence before an event to explain success of a system. For increasing the variety of values across cases, we had no theoretic justification to exclude any of the cases or remaining conditions or to rethink the coding of the conditions. We decided to add another case instead. We chose the system from Germany which was set up to monitor the O104:H4 outbreak in 2011. We have analysed this syndromic surveillance system in the framework of another study (28), and knew that it was qualifying for the QCA and that we could retrieve all necessary data from the broad publication coverage of this case. The consistency levels of the conditions were between 0.75 and 1.00, except for FREETEXT for which it was only 0.5. Thus, we decided to exclude FREETEXT from the minimisation process (table 3 ).

\section{Truth table and Boolean minimisation}

The cs/QCA steps of constructing the truth table and Boolean minimisation were accomplished using the software TOSMANA Version 1.302 (30).

\section{Syndromic influenza surveillance}

The truth table contained five configurations of the three conditions (table 3 ). Six cases were combined into two groups of configurations while the other three had individual configurations. The first round of Boolean minimisation including logical remainders was based on contradictory simplifying assumptions (CSA). That means that a logical remainder was used to explain both positive and negative outcome at the same time. This was resolved by applying the following procedure suggested by Delreux and Hesters (31). The CSA was assigned the likelier outcome of 1 according to case and theoretic knowledge and was included as logical remainder in the minimisation process for successful cases. The CSA was excluded as logical remainder from the minimisation process of the less likely outcome of 0 by including it as additional case.

\section{Situational awareness}

The truth table contained six configurations of the five factors (table 3 ). Three cases were combined in one configuration group while the other cases had individual configurations. The inclusion of logical remainders in the minimisation process did not lead to any contradictions. 
Table 3: Truth table for cs/QCA of seasonal syndromic influenza surveillance (a) and syndromic situational awareness (b)

\begin{tabular}{lllll}
$\begin{array}{l}\text { a) } \\
\text { NONCLIN }\end{array}$ & ACUTE & SUBNAT & Outcome & Cases \\
\hline 1 & 0 & 0 & 1 & 1177, GETWELL,NHS24 \\
0 & 1 & 1 & 1 & GENOA, SID CANT, SOSMEDECINS \\
1 & 0 & 1 & 1 & NHSDIRECT \\
0 & 0 & 1 & 0 & QSURVEILLANCE \\
0 & 1 & 0 & 0 & OSCOUR \\
0.57 & $0.43(0.75)$ & 0.57 & & Consistency (consistency only for cases NONCLIN $=0)$ \\
\hline
\end{tabular}

b)

\begin{tabular}{llllll} 
MULTIDATA & MULTISYND & AUTOM & EXIST & OUTCOME & Cases \\
\hline 0 & 0 & 1 & 1 & 0 & Denmark \\
1 & 1 & 1 & 1 & 1 & England, France, Scotland \\
0 & 1 & 0 & 0 & 0 & Italy Migrants \\
1 & 1 & 0 & 1 & 0 & SIDARTHa \\
0 & 1 & 1 & 1 & 1 & Sweden \\
0 & 0 & 0 & 0 & 0 & Germany \\
0.75 & 1.00 & 1.00 & 1.00 & & Consistency \\
\hline
\end{tabular}

$0=$ absent, 1 = present, ACUTE = acute care data sources, AUTOM = automated syndromic surveillance system components, EXIST $=$ syndromic surveillance system existed before monitored event, MULTDATA = multiple data sources, MULTSYND = multiple syndromes, NONCLIN $=$ non-clinical data sources, SUBNAT $=$ subnational analysis

\section{Results}

\section{Syndromic influenza surveillance}

More than $75 \%(n=7)$ of systems registered influenza-like-illness cases earlier compared to traditional surveillance systems (table 2). Timeliness ranged from -2.5 weeks in the SOS Medecins system to 2.0 weeks in the QSurveillance system with an average of -0.75 weeks and a median of -0.9 weeks. The minimisation process resulted in two different solution terms explaining $57 \%(n=4)$ and $43 \%(n=3)$ of the successful cases, and in two solution terms explaining the unsuccessful cases, each explaining $50 \%(n=1)$ of the cases (table 4). According to these solutions, successful syndromic surveillance systems for timely influenza case detection are analysing non-clinical data sources or are analysing acute data sources in combination with applying subnational data analysis. Unsuccessful syndromic surveillance systems in terms of timely influenza case detection are analysing clinical data sources in combination with either the analysis of non-acute care data sources or without applying subnational analysis. No solutions leading to success are necessary as the outcome also occurs in the absence of the solutions. But, the solutions are sufficient as the outcome always occurs when the solutions are present but there are also other solutions leading to the outcome. 


\section{Situational awareness}

The systems covered different kind of events ranging from one event in four cases to four events in two cases (table 2). Reporting time for the six successful systems was around one day and the cases were all very close to each other. For the two unsuccessful cases, reporting time was around 4 and 14 days. The minimisation process resulted in one solution term explaining all successful cases and two explaining $50 \%(n=2)$ and $75 \%$ $(n=3)$ of the unsuccessful cases respectively (table 4). According to the solutions, successful syndromic situational awareness systems are analysing multiple syndromes and are automated. Unsuccessful systems are analysing single syndromes and/or are not automated. The combination of analysing multiple syndromes in an automated system is a necessary condition as it is always present when the outcome occurs and the outcome does not occur when this condition is absent. The analysis of multiple data sources and the existence of the system before the event occurred were not identified as key influencing factors.

Table 4: Solution terms for explaining successful and unsuccessful seasonal syndromic influenza surveillance (a) and syndromic situational awareness (b)

a)

\begin{tabular}{|c|c|c|c|c|c|}
\hline Solution terms & Cases & RC & UC & SC & $\mathrm{C}$ \\
\hline NONCLIN+ & (1177,GETWELL,NHS24+NHSDIRECT) & 0.57 & 0.57 & & 1.0 \\
\hline ACUTE*SUBNAT & (GENOA+SID CANT, SOSMEDECINS) & $0.43(1.0)^{*}$ & $0.43(1.0)^{*}$ & & 1.0 \\
\hline$\rightarrow$ OUTCOME & & & & 1.0 & 1.0 \\
\hline nonclin*acute & (QSURVEILLANCE) & $0.5(0.5)^{*}$ & $0.5(0.5)^{*}$ & & 1.0 \\
\hline nonclin*subnat & (OSCOUR) & 0.5 & 0.5 & & 1.0 \\
\hline$\rightarrow$ outcome & & & & 1.0 & 1.0 \\
\hline
\end{tabular}

b)

\begin{tabular}{|c|c|c|c|c|c|}
\hline Solution terms & Cases & RC & UC & SC & C \\
\hline MULTSYND*AUTOM & (England,France,Scotland+Sweden) & 1.0 & 1.0 & & 1.0 \\
\hline$\leftarrow$ OUTCOME & & & & 1.0 & 1.0 \\
\hline multsynd+ & (Denmark+Germany) & 0.5 & 0.25 & & 1.0 \\
\hline autom & (Italy Migrants+SIDARTHa+Germany) & 0.75 & 0.5 & & 1.0 \\
\hline$\rightarrow$ outcome & & & & 1.0 & 1.0 \\
\hline
\end{tabular}

*coverage referring only to clinical cases, Capital letter $=$ presence, small letter $=$ absence, $+=$ logical OR, $*=$ logical $A N D$, ACUTE = acute care data sources, AUTOM = automated syndromic surveillance system components, C = consistency, NONCLIN = non-clinical data sources, MULTSYND = multiple syndromes, RC = raw coverage (number of cases covered by solution of all cases with the same outcome), SC = solution coverage (number of cases covered by all solutions of all cases with the same outcome), SUBNAT = subnational analysis, UC = unique coverage (number of cases uniquely covered by a solution of all cases with the same outcome) 


\section{Discussion}

Using cS/QCA we compared the performance and identified success factors of different European syndromic surveillance systems for two major areas of application. We found that syndromic influenza surveillance might be timelier when analysing non-clinical data sources. For timely situational awareness during various kinds of events, we identified the analysis of multiple syndromes in automated syndromic surveillance systems as a necessary condition for success.

\section{Interpretation}

\section{Syndromic influenza surveillance}

The low coverage levels of the solutions reflect the low consistency level of the data and indicate a need for a careful interpretation of the solutions and a limitation of their practical relevance. The solution for successful systems confirmed the hypothesis that the analysis of non-clinical data can support timely seasonal infectious disease surveillance. The analysis of acute care data sources seems to be of lesser importance and only in combination with another condition for timeliness such as subnational analysis.

Only clinical data based cases were unsuccessful. One of the two unsuccessful cases, QSurveillance, is based on data from general practitioners. In the course of illness or patient treatment, this data source is positioned closer to the traditional data sources of confirmed diagnoses from sentinel general practitioners or laboratories. Compared to the non-clinical data sources timeliness of general practitioner data sources must be lower. The timeliness of the second system, Oscour, which is based on emergency department data was referring to two influenza seasons in 2005 and 2006. As the system was used since then on a regular basis for influenza surveillance in France, it might as well have shown better timeliness in other seasons comparable to the other acute care data source based systems that we analysed.

While the analysis of subnational data was expected to be supportive, the analysis of age groups does not seem to have an added value. However, that does not say that subnational or age-adjusted analysis does not lead to timelier surveillance, it might just not be applied in many systems. QCA does say more about necessary or sufficient causes and combinations thereof in relation to each other than about the potential of single success factors (29).

When looking at applications of the analysed syndromic surveillance systems for other infectious disease outbreaks, the two solutions explaining successful outcome are supported. For detection of seasonal gastrointestinal outbreaks, the NHS Direct system in England could achieve a timeliness of -5.68 weeks on average by using non-clinical data (32). No subnational analysis was applied but analysis by age-group. The syndromic surveillance system in Genoa monitored two measles outbreaks with a timeliness of -3.57 weeks on average based on acute care data $(33,34)$. In this case, subnational surveillance was applied in one study and was connected to a 1.43 week advance warning (34). Age-adjusted analysis was not applied. We could only collect data on syndromic surveillance of other infectious disease outbreaks for these two systems and therefore they can only function as indication for a confirmation of our results.

Looking into other systems outside Europe for syndromic seasonal influenza surveillance, we can best look into a review by Dailey and colleagues comparing different systems and 
data sources for timeliness of syndromic seasonal influenza surveillance (11). The authors also found a general higher timeliness of non-clinical and acute care data sources. When looking into the single studies included in the review, there were no comparable nonclinical data based systems but six systems based on emergency department data. These are comparable to the acute care systems included in our study. One of these systems was associated with a negative outcome according to our definition but this system also applied subnational analysis (35). Another system applied subnational analysis which was associated with a positive outcome (36). Three other successful systems did not apply subnational analysis (37-39), one applied spatial cluster analysis but did not find any clusters associated with influenza (40) and one also applied age-adjusted analysis (39). From the six systems, five only reported results for one influenza season which reduces the representativeness of the results $(35-37,39,40)$. The results of emergency department data based systems included in our study were all based on more than one season. The sixth study did not compare syndromic with traditional surveillance results which is limiting the comparability to our results (38). For these emergency department data based systems we could not retrieve any further publications focusing on syndromic influenza surveillance reporting exact timeliness measurements.

The comparison with other systems indicates that the two conditions associated with data sources might be success factors for syndromic influenza surveillance with a trend towards non-clinical data sources. The necessity of subnational analysis in combination with acute care data sources for success might be weaker. Future QCA including other systems or further data on the included systems are expected to add to the evidence.

\section{Situational awareness}

The possibility to analyse multiple syndromes and the use of automated systems were prominent success factors which is in line with our hypothesis. Even more, the combination of these factors was identified as a necessary condition for success explaining all observed successful cases. The absence of one or both of these conditions was connected with unsuccessful syndromic situational awareness.

To our surprise, that means against the expectations formulated during the site visits, the condition of analysing multiple data sources turned out not to be a success factor for flexible situational awareness. Multiple data sources were analysed in successful and unsuccessful systems. Further, successful as well as unsuccessful systems existed before the event. Therefore, this condition could not be identified as success factor. However, all successful systems were established before the event giving some indication that this can be a characteristic of successful systems.

Systems outside Europe which were used only for one event share similar characteristics as the unsuccessful systems in our study. For example, systems that were set up 2005 in the aftermath of hurricane Katrina in different cities in the United States of America (USA) were analysing multiple databases and multiple syndromes daily. But, they were not existing before the event, and were based on manual data collection and analysis $(41,42)$. The system set up for monitoring the Kentucky Derby in Louisville, USA, for at least the years 2002-2004 was neither automated nor established prior to the event (and terminated between events). But, it was monitoring multiple syndromes on a daily basis (43). Also in these examples for unsuccessful systems, the combination of multiple syndrome analysis and automation was absent as suggested by the QCA solution. 
Turning to successful systems outside Europe, one long established system that was used during different events is the system in New York City, USA. Next to multiple other data sources, especially emergency department data were used for situational awareness (44). The emergency department data based analysis started as an ad-hoc and non-automated setup in the days after the September 2011 terrorist attacks $(45,46)$. The system was maintained by changing it into an automated system which again shows the importance of automation as a key success factor. The automated emergency department data based system was used to monitor multiple syndromes during the blackout in 2003 (47), the A/H1N1 pandemic $2009(48,49)$ and the effects of hurricane Sandy in $2012(50)$. Another system part which was analysing multiple other data sources was only used for monitoring one syndrome during the blackout in 2003 (47). This system part seemed to be automated for certain data sources but the information we could obtain from the literature was unambiguous. For at least one part of the New York City system, the combination of multiple syndrome analysis in an automated system is connected with successful situational awareness. The example also confirms that the use of multiple data sources is not a key success factor.

\section{Limitations}

The low quality of the data table and solutions of the QCA regarding seasonal infectious disease surveillance shows the importance of the quality of the data input for QCA. We tried to include representative data providing results for more than one influenza season. We decided to include two systems despite results referred only to one season: NHS24 in Scotland and QSurveillance in England. NHS24 was applied in more than one influenza season and reported timeliness data were positive but not explicitly quantified for the other seasons (51). As QSurveillance was the only case representing a primary care data based system, we decided to include it to add variety to the QCA. The QSurveillance system was changed during the influenza season for which the results were reported, so performance could also change in the future (52). The results for QSurveillance are to be interpreted carefully and transfer to other syndromic surveillance systems based on general practitioner data might not be validated.

The conditions analysed in the QCA were chosen based on theoretic considerations but also based on the available data. There might be other conditions that we did not include in our analysis such as validity or representativeness, or other cases based on other data sources which might play an important role. But, we could not include them due to limited data availability or in the case of validity because of the large diversity in measuring this indicator in the different surveillance systems.

QCA makes it necessary to differentiate successful from unsuccessful cases. We defined the cut-off points for both analyses based on the results reported for the cases and theoretic considerations. The low number of unsuccessful cases with a limited representativeness included in the QCA on infectious disease surveillance might have limited our options to define a representative cut-off. Furthermore, the distinction of successful or unsuccessful cases should not be generalised to the syndromic surveillance systems behind these cases. The syndromic surveillance systems might perform differently when they are monitoring other syndromes, for example.

Finally, the data in our study were not collected with QCA in mind. We chose the method afterwards to add value to our complex case-based data. With QCA in mind, we might 
have collected different or additional data which could have improved quality of the input and output of the QCA.

\section{Conclusions}

We identified key success factors for the two main areas of syndromic surveillance system application using cs/QCA. For syndromic influenza surveillance, a system might be timelier if it is based on non-clinical data sources. Syndromic situational awareness is fostered by automated syndromic surveillance systems capable of analysing multiple syndromes. Analysing multiple data sources is no pre-requisite for successful situational awareness. We recommend that decision makers take the success factors into account when designing or further developing a syndromic surveillance system.

We showed that the relatively new social science analysis method QCA can have a relevant added value for public health surveillance practice by comparing case-based, small-N and mixed data in a systematic way. QCA can only produce valid results if it is guided by good case and subject expertise and if it is based on varied and representative data. We propose to apply QCA to other case-based and small-N data next to more traditional analysis methods as it might yield meaningful and relevant results for policy and practice that would otherwise remain undiscovered.

\section{Acknowledgements}

This paper arises from the project Triple S-AGE which has received funding from the European Union, in the framework of the Health Programme (grant agreement number 20091112). 


\section{References}

1. Triple S Project. Assessment of syndromic surveillance in Europe. Lancet. 2011;378(9806):1833-4.

2. Ziemann A, Krafft T. Guidelines for Assessment of Data Sources; 2013 [cited 28 May 2015]. Available from: http://www.syndromicsurveillance.eu/tripleS_guidelines_datasources.pdf.

3. Fouillet A, Sala-Soler M, Conti S, Kanieff M, Rago G, Perrin JB, et al. Inventory of syndromic surveillance systems in Europe by the Triple-S project. Emerg Health Threats J. 2011;4:10946.

4. Koopmans M. Surveillance strategy for early detection of unusual infectious disease events. Curr Opin Virol. 2013;3(2):185-91.

5. Kaydos-Daniels SC, Rojas Smith L, Farris TR. Biosurveillance in outbreak investigations. Biosecur Bioterror. 2013;11(1):20-8.

6. Buehler JW, Whitney EA, Smith D, Prietula MJ, Stanton SH, Isakov AP. Situational uses of syndromic surveillance. Biosecur Bioterror. 2009;7(2):165-77.

7. Paterson BJ, Durrheim DN. The remarkable adaptability of syndromic surveillance to meet public health needs. J Epidemiol Glob Health. 2013;3(1):41-7.

8. Buehler JW, Hopkins RS, Overhage JM, Sosin DM, Tong V. Framework for evaluating public health surveillance systems for early detection of outbreaks: recommendations from the CDC Working Group. MMWR Recomm Rep. 2004;53(RR-5):1-11.

9. Triple S Project. Guidelines for designing and implementing a syndromic surveillance system. Triple S Project; 2013 [cited 28 May 2015]. Available from: http://www.syndromicsurveillance.eu/Triple-S_guidelines.pdf.

10. Rosenkötter N, Ziemann A, Riesgo LG, Gillet JB, Vergeiner G, Krafft T, et al. Validity and timeliness of syndromic influenza surveillance during the autumn/winter wave of A(H1N1) influenza 2009: results of emergency medical dispatch, ambulance and emergency department data from three European regions. BMC Public Health. 2013;13(1):905. doi: 10.1186/1471-2458-13-905.

11. Dailey $L$, Watkins RE, Plant AJ. Timeliness of data sources used for influenza surveillance. J Am Med Inform Assoc. 2007;14(5):626-31.

12. Doroshenko A, Cooper D, Smith G, Gerard E, Chinemana F, Verlander N, et al. Evaluation of syndromic surveillance based on National Health Service Direct derived data--England and Wales. MMWR Morb Mortal Wkly Rep. 2005;54 Suppl:117-22.

13. Jefferson H, Dupuy B, Chaudet H, Texier G, Green A, Barnish G, et al. Evaluation of a syndromic surveillance for the early detection of outbreaks among military personnel in a tropical country. J Public Health (Oxf). 2008;30(4):375-83.

14. Ragin CC. The comparative comparative analysis to study causal complexity. Health Serv Res. 1999;34(5 Pt 2):1225-39.

15. Ragin CC. What is Qualitative Comparative Analysis (QCA)? 2008 [cited 28 May 2015]. Available from: http://eprints.ncrm.ac.uk/250/1/What_is_QCA.pdf. 
16. Rihoux B, De Meur G. Crisp-Set Qualitative Comparative Analysis (csQCA). In: Rihoux B, Ragin CC, editors. Configurational Comparative Methods: Qualitative Comparative Analysis (QCA) and Related Techniques. Thousand Oaks: SAGE; 2009. p. 33-68.

17. Blackman T, Wistow J, Byrne D. A Qualitative Comparative Analysis of factors associated with trends in narrowing health inequalities in England. Soc Sci Med. 2011;72(12):1965-74.

18. Thomas J, O'Mara-Eves A, Brunton G. Using qualitative comparative analysis (QCA) in systematic reviews of complex interventions: a worked example. Syst rev. 2014;3:67. doi: 10.1186/2046-4053-3-67.

19. Warren J, Wistow J, Bambra C. Applying qualitative comparative analysis (QCA) in public health: a case study of a health improvement service for long-term incapacity benefit recipients. J Public Health (Oxf). 2014;36(1):126-33.

20. Thiem A, Dusa A. Boolean Minimization in Social Science Research: A Review of Current Software for Qualitative Comparative Analysis (QCA). Soc Sci Comput Rev. 2013;31(4):505-21.

21. Triple S-AGE [Internet]; 2015 [cited 28 May 2015]. Available from: www.syndromicsurveillance.eu.

22. Conti S, Kanieff M, Rago G, on behalf of the Triple-S project. Inventory of Syndromic Surveillance Systems in Europe; 2012 [cited 28 May 2015]. Available from: http://www.syndromicsurveillance.eu/images/stories/Final_material/tripleS_country_visits.pdf

23. Ziemann A, Krafft T, Sala Soler M, Sypniewska P. Country visits; 2013 [cited 28 May 2015]. Available from: http://syndromicsurveillance.eu/images/stories/Final_ material/triple-S_country_visits.pdf

24. Robert Koch Institut. Final presentation and evaluation of epidemiological findings in the EHEC 0104:H4 Outbreak, Germany 2011. Berlin: Robert Koch-Institute; 2011.

25. Wadl M, Rieck T, Nachtnebel M, Greutelaers B, an der Heiden M, Altmann D, et al. Enhanced surveillance during a large outbreak of bloody diarrhoea and haemolytic uraemic syndrome caused by Shiga toxin/verotoxin-producing Escherichia coli in Germany, May to June 2011. Euro Surveill. 2011;16(24). pii: 19893.

26. Mandl KD, Overhage JM, Wagner MM, Lober WB, Sebastiani P, Mostashari F, et al. Implementing syndromic surveillance: a practical guide informed by the early experience. J Am Med Inform Assoc. 2004;11(2):141-50.

27. Rosenkötter N, Ziemann A, Krafft T, Riesgo LG, Vergeiner G, Brand H. Non-infectious events under the International Health Regulations (2005) in Europe - a case for syndromic surveillance. J Public Health Policy. 2014;35(3):311-26.

28. Ziemann A, Rosenkötter N, Garcia-Castrillo Riesgo L, Fischer M, Krämer A, Lippert FK, et al. Meeting the International Health Regulations (2005) surveillance core capacity requirements at the subnational level in Europe: the added value of syndromic surveillance. BMC Public Health. 2015 Feb 7;15:107. doi: 10.1186/s12889-015-1421-2.

29. Schneider C, Wagemann C. Standards of Good Practice in Qualitative Comparative Analysis (QCA) and Fuzzy-Sets. Comp Sociol. 2010;9:397-418. 
30. Cronqvist L. Tool for Small-N Analysis (Version 1.302). Trier: Universität Trier; 2011. Available from: http://www.compasss.org/software.htm\#tosmana.

31. Delreux T, Hesters D. Solving contradictory simplifying assumptions in QCA: presentation of a new best practice. 2010;58:1-27 [cited 28 May 2015]. Available from: http://www.compasss.org/wpseries/DelreuxHesters2010.pdf.

32. Loveridge P, Cooper D, Elliot AJ, Harris J, Gray J, Large S, et al. Vomiting calls to NHS Direct provide an early warning of norovirus outbreaks in hospitals. J Hosp Infect. $2010 ; 74(4): 385-93$.

33. Ansaldi F, Orsi A, Altomonte F, Bertone G, Parodi V, Carloni R, et al. Syndrome surveillance and molecular epidemiology for early detection and tracing of an outbreak of measles in Liguria, Italy. J Med Virol. 2009;81(10):1807-13.

34. Orsi A, Alicino C, Patria AG, Parodi V, Carloni R, Turello V, et al. Epidemiological and molecular approaches for management of a measles outbreak in Liguria, Italy. J Prev Med Hyg. 2010;51(2):67-72.

35. Ritzwoller DP, Kleinman K, Palen T, Abrams A, Kaferly J, Yih W, et al. Comparison of syndromic surveillance and a sentinel provider system in detecting an influenza outbreak--Denver, Colorado, 2003. MMWR Morb Mortal Wkly Rep. 2005;54 Suppl:151-6.

36. Yuan CM, Love S, Wilson M. Syndromic surveillance at hospital emergency departments--southeastern Virginia. MMWR Morb Mortal Wkly Rep. 2004;53 Suppl:56-8.

37. Irvin CB, Nouhan PP, Rice K. Syndromic analysis of computerized emergency department patients' chief complaints: an opportunity for bioterrorism and influenza surveillance. Ann Emerg Med. 2003;41(4):447-52.

38. Ivanov O, Gesteland PH, Hogan W, Mundorff MB, Wagner MM. Detection of pediatric respiratory and gastrointestinal outbreaks from free-text chief complaints. AMIA Ann Symp Proc. 2003:318-22.

39. Miller B, Kassenborg H, Dunsmuir W, Griffith J, Hadidi M, Nordin JD, et al. Syndromic surveillance for influenzalike illness in ambulatory care network. Emerg Infect Dis. 2004;10(10):1806-11.

40. Heffernan R, Mostashari F, Das D, Karpati A, Kulldorff M, Weiss D. Syndromic surveillance in public health practice, New York City. Emerg Infect Dis. 2004;10(5):858-64.

41. Murray KO, Kilborn C, DesVignes-Kendrick M, Koers E, Page V, Selwyn BJ, et al. Emerging disease syndromic surveillance for Hurricane Katrina evacuees seeking shelter in Houston's Astrodome and Reliant Park Complex. Public Health Rep. 2009;124(3):364-71.

42. Centers for Disease Control and Prevention. Injury and illness surveillance in hospitals and acute-care facilities after Hurricanes Katrina And Rita--New Orleans area, Louisiana, September 25-October 15, 2005. MMWR Morb Mortal Wkly Rep. 2006;55(2):35-8. 
43. Carrico R, Goss L. Syndromic surveillance: hospital emergency department participation during the Kentucky Derby Festival. Disaster Manag Response. 2005;3(3):73-9.

44. Heffernan R, Mostashari F, Das D, Besculides M, Rodriguez C, Greenko J, et al. New York City syndromic surveillance systems. MMWR Morb Mortal Wkly Rep. 2004;53 Suppl:23-7.

45. Centers for Disease Control and Prevention. Syndromic surveillance for bioterrorism following the attacks on the World Trade Center--New York City, 2001. MMWR Morb Mortal Wkly Rep. 2002;51 Spec No:13-5.

46. Das D, Weiss D, Mostashari F, Treadwell T, McQuiston J, Hutwagner L, et al. Enhanced drop-in syndromic surveillance in New York City following September 11, 2001. J Urban Health. 2003;80(2 Suppl 1):i76-88.

47. Marx MA, Rodriguez CV, Greenko J, Das D, Heffernan R, Karpati AM, et al. Diarrheal illness detected through syndromic surveillance after a massive power outage: New York City, August 2003. Am J Public Health. 2006;96(3):547-53.

48. Plagianos MG, Wu WY, McCullough C, Paladini M, Lurio J, Buck MD, et al. Syndromic surveillance during pandemic (H1N1) 2009 outbreak, New York, New York, USA. Emerg Infect Dis. 2011;17(9):1724-6.

49. Hadler JL, Konty K, McVeigh KH, Fine A, Eisenhower D, Kerker B, et al. Case fatality rates based on population estimates of influenza-like illness due to novel H1N1 influenza: New York City, May-June 2009. PLoS One. 2010;5(7):e11677. doi: 10.1371/journal. pone.0011677.

50. Chen BC, Shawn LK, Connors NJ, Wheeler K, Williams N, Hoffman RS, et al. Carbon monoxide exposures in New York City following Hurricane Sandy in 2012. Clin Toxicol (Phila). 2013;51(9):879-85.

51. Kavanagh K, Robertson C, Murdoch H, Crooks G, McMenamin J. Syndromic surveillance of influenza-like illness in Scotland during the influenza A H1N1v pandemic and beyond. J R Stat Soc a Stat. 2012;175:939-58.

52. Green HK, Zhao H, Boddington NL, Andrews N, Durnall H, Elliot AJ, et al. Detection of varying influenza circulation within England in 2012/13: informing antiviral prescription and public health response. J Public Health (Oxf). 2015;37(2):295-304. 

CHAPTER 7

General Discussion 


\section{Main findings \& discussion}

\section{Added value of syndromic surveillance for Europe}

First of all, this thesis assessed the added value of syndromic surveillance for Europe as one way to improve Europe's surveillance capacity. In a first step, a European syndromic surveillance definition and a system concept were presented. The system concept is balancing the European principals of harmonisation, mutual recognition and subsidiarity. Secondly, the thesis showed that syndromic surveillance is useful for Europe in terms of providing timely and additional information on various kinds of health threats. Nonspecificity applies to certain data sources, syndromes or events but is not a general weakness of syndromic surveillance. And finally, the thesis showed that syndromic surveillance can support the implementation of the core surveillance capacity requirements of the International Health Regulations (2005) (IHR (2005)). Syndromic surveillance can provide timely information at the subnational level for various health threats as required by the IHR (2005). Figure 1 summarises the results of the thesis that are discussed in the following.

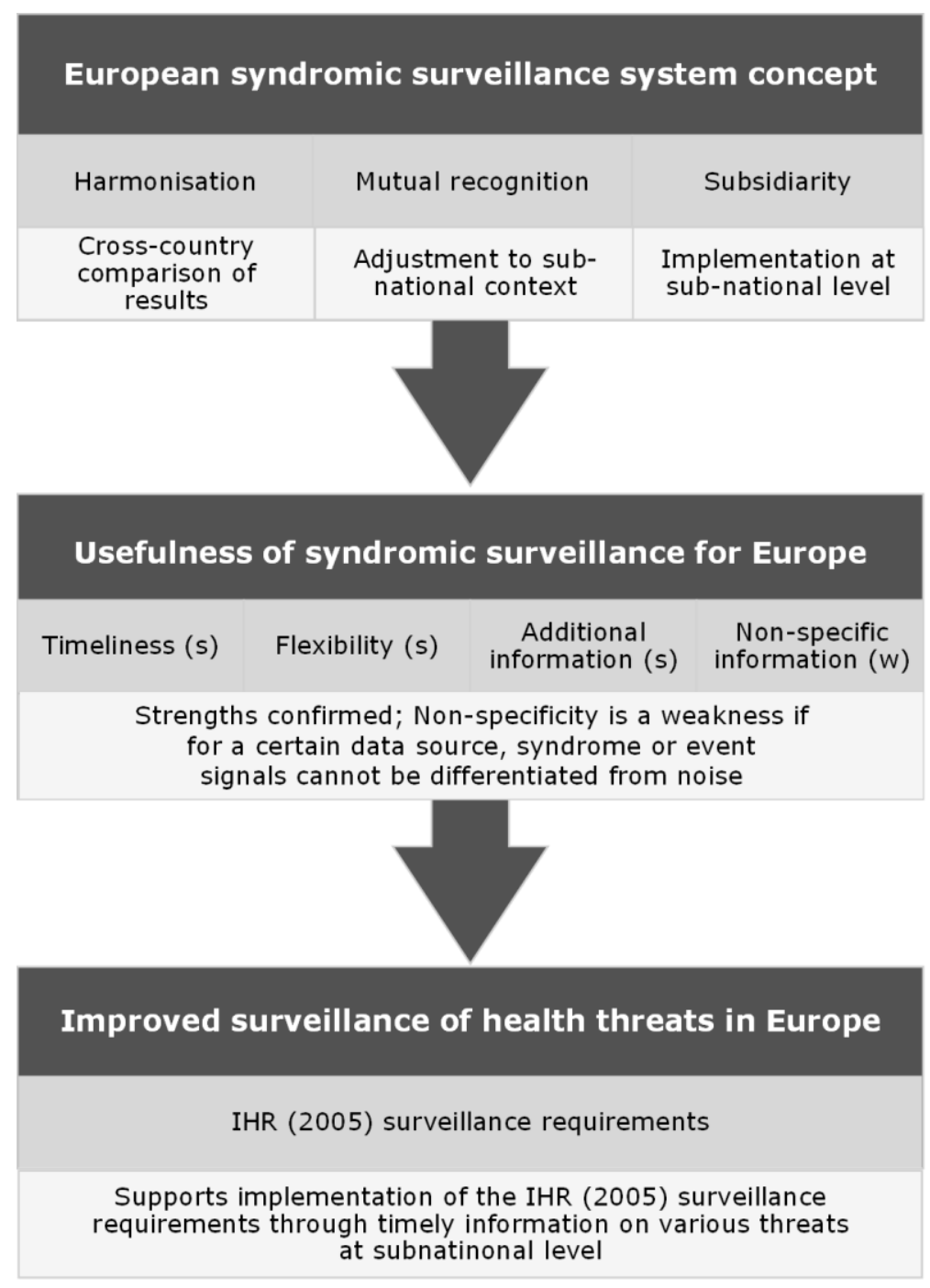

Figure 1: Summary of added value of syndromic surveillance for Europe (own concept) IHR (2005) = International Health Regulations (2005) $s=$ strength, $w=$ weakness 
In chapter 2, a new syndromic surveillance definition was presented which is not only valid in the European context. The updated definition is more comprehensive and generic than the earlier definition by the Centers for Disease Control and Prevention (1). It covers human and animal health, various data sources and features the main advantages of syndromic surveillance such as augmenting traditional surveillance systems, timeliness and flexibility. The focus on automated data collection is in line with the results presented in chapter 6 identifying automation as a key characteristic of successful syndromic surveillance systems. Until now, the definition was cited in 21 scientific articles according to ScienceDirect (2).

In chapters 3 and 5, a concept for a European syndromic surveillance system was presented. Chapter 3 focused on the design of the system while chapter 5 presented the concept for its implementation in the European context. The system balances between the principles of subsidiarity, mutual recognition, and harmonisation. Instead of recommending one harmonised European syndromic surveillance system, the concept suggests to implement a network of subnational systems. They would be embedded into the existing surveillance structure of a country, thus supporting the principle of subsidiarity. The surveillance results were anticipated to still be comparable across systems and borders. This would allow for a pragmatic balance between harmonisation and mutual recognition across Europe. As shown in chapter 4, the SIDARTHa concept performed best if adjusted to local or regional circumstances. This fosters mutual recognition of the system designs in the different countries. As chapter 5 showed, the concept is implemented in two regions in Europe at the moment, indicating its applicability in the European context. However, in order to assess the real European added value, the output of many more subnational syndromic surveillance systems in Europe needs to be assessed. Otherwise, the public health impact of an event might not be assessable for larger populations.

Chapters 3-6 assessed the usefulness of syndromic surveillance for Europe by focussing on its main purposes: surveillance of (seasonal) influenza and gastrointestinal outbreaks, and situational awareness during various events. The main strength of syndromic surveillance, timeliness, was confirmed when assessing cases for all three purposes. This became apparent especially for seasonal influenza surveillance (chapters 4,6) and for situational awareness of diverse health threats (chapters 5, 6). As chapter 6 showed, timeliness of syndromic surveillance for seasonal influenza surveillance might be increased when non-clinical data sources are analysed. The results are in line with the only other study comparing multiple systems from Dailey and colleagues (3). They found that syndromic surveillance systems provided timelier information on seasonal influenza surveillance. To my knowledge, the results presented in chapters 5 and 6 are the only other studies comparing timeliness of multiple syndromic surveillance systems.

The other main advantage of syndromic surveillance is flexibility in terms of applicability to various, including unexpected events. This strength was confirmed for situational awareness during events as diverse as the 2009 influenza pandemic, the 2010 volcanic ash plume or the influx of migrants from North Africa to Italy in 2011 (chapters 5, 6). This flexibility can also provide a useful and cost-effective support to fulfil the surveillance requirements of the IHR (2005) (chapter 5). Flexibility of syndromic surveillance can be enhanced if multiple syndromes are analysed in automated systems as shown in chapter 6 . The reviews presented in chapters 5 and 6 are to my knowledge the only studies comparing performance of multiple syndromic surveillance systems for situational 
awareness. The only other review looking into flexibility of syndromic surveillance published by Paterson et al. did not compare systems by performance but showed general changes in syndromic surveillance application over time (4).

Syndromic surveillance is especially useful at times when it can provide additional information on the public health impact of an event. This was confirmed in the case study presented in chapter 4 . Here, syndromic surveillance was the only source of information during the Christmas holidays when the traditional surveillance system based on sentinel general practitioners was not active. Also the examples presented in chapter 5 confirmed this for the 2011 O104:H4 gastrointestinal outbreak in Germany. During this outbreak, traditional surveillance was delayed and scattered (5). The other example from chapter 5 is the 2010 volcanic ash plume event. During this event, syndromic surveillance systems were the only source of information on the potential health impact of the plume.

As the case study in chapter 3 showed, syndromic surveillance can also provide additional information on population groups that are not monitored by traditional surveillance. In this case, syndromic surveillance covered a group of foreign tourists. This is also the idea behind syndromic surveillance systems covering non-clinical data sources, such as web searches, over-the-counter sales or telephone helplines. The aim is to detect outbreaks in a population which does not (yet) seek health care. People might have milder symptoms and chose self-treatment instead. Especially for gastrointestinal outbreaks which are often characterised by mild symptoms, syndromic surveillance of non-clinical data sources showed promising results to augment traditional surveillance $(6,7)$. Also the opposite, severe gastrointestinal cases treated in the acute care setting, can be covered by syndromic surveillance. As shown in chapter 5 , a syndromic surveillance system was set up during the 0104:H4 outbreak in Germany to monitor severe cases in emergency departments.

Non-specificity remains a major weakness of syndromic surveillance. But, its negative impact differs depending on the analysed syndrome, data source or event. As demonstrated during the case study presented in chapter 3, point-source gastrointestinal outbreaks were not identified by syndromic surveillance. This was basically because signals could not be differentiated from the background noise. There are various reasons related to non-specificity which can explain this effect. The size of the outbreak in terms of the number of cases must be large enough to be differentiated from the usual number of cases expected at a certain time. Other studies confirmed these findings. Outbreaks with a small number of cases were not detected as often as outbreaks with large case numbers $(6,8)$. Further, outbreaks consisting of spatially dispersed cases were difficult to be detected by syndromic surveillance as chapter 3 showed. Also the choice of the data source for syndromic surveillance can influence the impact of non-specificity. As discussed above, gastrointestinal illness is often characterised by mild syndromes. Patients might not seek emergency but primary care or self-treatment. Thus, gastrointestinal outbreaks might be easier distinguished from noise in non-emergency care data sources. As Rosenkötter et al. showed with data from the SIDARTHa action, the choice of data source made a difference also for the validity of seasonal influenza surveillance. In their study, seasonal influenza cases could not be distinguished from noise using emergency medical dispatch data but very well using emergency department data (9).

Finally, chapter 5 assessed the potential of syndromic surveillance to improve Europe's surveillance capacity according to the IHR (2005) requirements. Syndromic surveillance is considered to help detect and assess the local and regional effect of different types of 
public health emergencies in a timely manner as required by the IHR (2005). This might especially hold true for rare and non-infectious health threats for which no (timely) information on the public health impact is available from other systems. One example was the volcanic ash plume event in 2010 (chapter 5). Also Rosenkötter et al. argued for the potential of syndromic surveillance to support countries to fulfil the IHR (2005) requirements regarding monitoring of non-infectious events (10).

\section{Added value of Europe for syndromic surveillance}

The thesis also analysed the added value of Europe for gaining new knowledge about syndromic surveillance. Chapters 2-6 presented new knowledge on the usefulness of syndromic surveillance. The results were based on the work accomplished in the two European Health Programme actions SIDARTHa and Triple S-AGE. Especially, chapter 6 showed that new knowledge could be derived from a comparative analysis of syndromic surveillance systems from different European countries. The chapter identified key success factors for syndromic surveillance systems. These can inform decision makers in Europe and beyond when setting up or further developing syndromic surveillance systems.

The relatively new social science approach Qualitative Comparative Analysis was useful for generating new knowledge as shown in chapter 6 . The particularity of the approach was the structured analysis of small- $\mathrm{N}$, case-based and mixed data from different countries. Cross-country comparisons usually analyse quantitative data such as in the work of the WHO European Observatory on Health Systems and Policy (11). Qualitative Comparative Analysis might be a promising approach to enhance cross-country comparisons with a structured analysis approach for other kinds of data. Until now, Qualitative Comparative Analysis was mainly applied in the political sciences. The few health-related studies applying Qualitative Comparative Analysis were often analysing the situation in single countries $(12,13)$. There are only few other examples of studies using Qualitative Comparative Analysis for cross-country comparisons in the area of health (1417). To my knowledge, there are only two other studies comparing European countries $(18,19)$.

The learning process on syndromic surveillance in Europe can be described using the conceptual framework for cross-country learning in Europe presented in chapter 1 (figure 2). The first dimension of 'Learning in Europe' comprises the country visits for knowledge exchange and the inventory of syndromic surveillance systems of the Triple S-AGE action (20-22). Further it entails the inventory accomplished in the framework of the SIDARTHa action (chapter 3 ). The activities in this category are more descriptive. In the case of the country visits the impact in terms of knowledge gain in the participating countries is difficult to measure. Part of the second dimension 'Learning for Europe' is the syndromic surveillance definition presented in chapter 2. Also the European syndromic surveillance system concept presented in chapters 3 and 5 can be sorted into this category. Further, the Triple S-AGE action developed guidelines and a European strategy for syndromic surveillance which fit in this dimension $(23,24)$. The Triple S-AGE guidelines and the strategy provide a balance between European harmonisation and diversity by suggesting different modes of syndromic surveillance implementation. As described above, the SIDARTHa concept fits to the European principles of harmonisation, mutual recognition and subsidiarity. These concrete concepts and tools can now be applied in different contexts in Europe. The SIDARTHa concept was implemented in two 
regions and tested in different case studies described in chapters 3 and 4 and by Rosenkötter et al $(25,26)$. The dimension 'Learning from Europe' refers to the Qualitative Comparative Analysis of syndromic surveillance systems presented in chapter 6 . New knowledge on syndromic surveillance was gained from a structured cross-country comparison in Europe. This knowledge can now be implemented in different contexts in Europe and beyond.

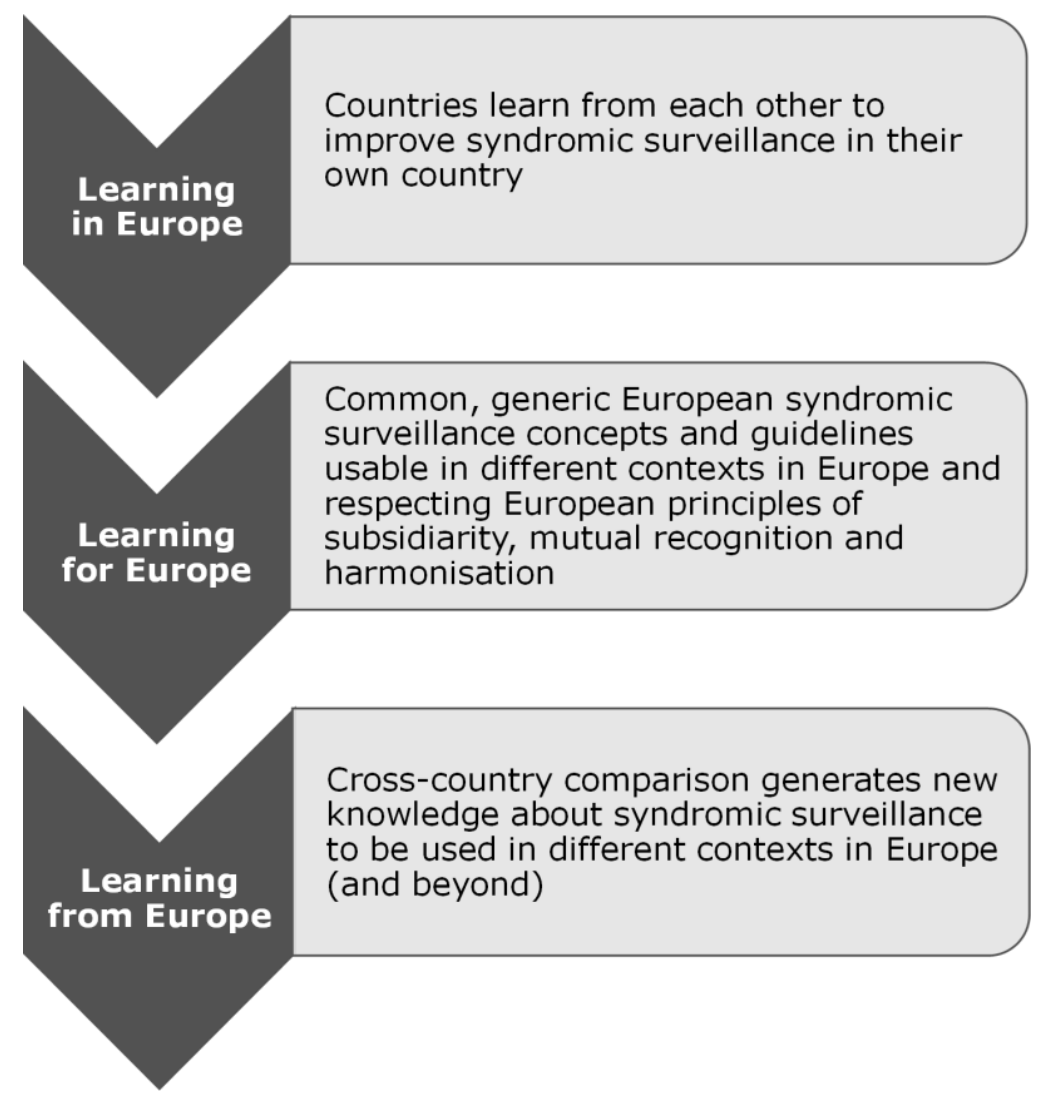

Figure 2: Cross-country learning about syndromic surveillance in the European context (own concept)

\section{Limitations}

\section{Added value of syndromic surveillance for Europe}

The syndromic surveillance concept was assessed for its European fit according to the European principles harmonisation, mutual recognition and subsidiarity. These principles were a selection of a couple of mainly legal principles that are underlying the functioning of the European Union (EU) (27). The other principles such as country of origin for products or precedence and direct effect of EU law were not applicable to the interventions analysed here. The use of the principles to describe the European fit was meant as a conceptual framework for the work in this thesis. It is not a proposal for the assessment or evaluation of EU actions. Future work might show if the principles are suitable for describing the European fit of other actions.

The assessment of the usefulness of syndromic surveillance in Europe was based on a selection of health threats and performance indicators. The health threats are the major purposes of syndromic surveillance. But, there are other syndromes and health threats which are monitored by syndromic surveillance. Examples comprise other infectious 
diseases such as measles (28), or non-infectious health problems such as poisonings (29). For many years, the approach has also used as a substitute for traditional surveillance in developing countries (30). And, there are many syndromic surveillance applications based on animal health data that were not included in the present research (22). Especially for the use in the comparative studies presented in chapters 5 and 6, the selection of health threats was also based on data availability.

The performance indicators are considered to be the key strengths and weakness of syndromic surveillance. However, there are many more indicators for evaluating syndromic surveillance systems (1). For example, cost-effectiveness is considered another advantage of syndromic surveillance but there is hardly any data available on this indicator $(31,32)$. Also other indicators related to experience of syndromic surveillance systems such as acceptability or stability are not often described in syndromic surveillance evaluations (32-34). Validity on the other hand is reported in many cases, also in those presented in chapters 3 and 4 . However, the indicator was excluded from the comparative studies in chapters 5 and 6 . Validity was measured in so many different ways that it made a comparison a too complex endeavour. In the future, there might be more evaluations reporting on other indicators to include them in comparative studies.

Syndromic surveillance might support the implementation of the IHR (2005) core surveillance capacity requirements. This suggestion was only focusing on the requirement of providing immediate information on various health threats. The implementation of the IHR (2005) or its surveillance requirements in Europe can depend on many other factors, the availability of resources for example (35). There might also be other measures that help countries improve their capacity to monitor diverse health threats. One example is enhancing traditional surveillance as it was done during the FIFA Worldcup in Germany 2006, for example (36). Another strategy is to set up ad-hoc surveillance systems during unfolding events. Such systems can be of added value as seen during the 0104:H4 gastrointestinal outbreak in Germany, for example (39). However, as chapters 5 and 6 showed, such ad-hoc systems might also be prone to delayed reporting, lower validity and higher costs compared to already established syndromic surveillance systems.

\section{Added value of Europe for syndromic surveillance}

The proposed conceptual framework for cross-country learning in Europe is a first attempt to capture cross-country learning in the unique European context, the proclaimed 'laboratory' (38). The concept was tested only for the knowledge gained in EU Health Programme actions. There might be other kinds of European cross-country learning beyond EU Health Programme actions that could be included in the concept. For example, there might be unique European situations or events with a health impact such as the recent financial crisis (39). These events might yield lessons that can only be learnt in the European context. This example might fit into the third dimension of Yearning from Europe' in the proposed conceptual framework.

Another question that arises is if the generation of new knowledge as described in the conceptual framework is only possible in the European context. The conceptual framework might be applicable also to learning processes involving countries and regions outside Europe. What this thesis showed is that the European context definitely provided a unique opportunity to gain new knowledge on syndromic surveillance. There is a legal framework to collaborate in the fight against health threats with the Decision on health 
threats (40). There is the political will and financial support of the EU to support crosscountry learning in the framework of the Health Programme. And finally, collaboration of European countries in such actions might be easier through joint aims, values and already existing linkages in many areas.

\section{Implications for policy and practice \& future research}

Despite the implementation of many syndromic surveillance systems, there is still doubt about the usefulness of the approach among many public health professionals and decision makers $(41,42)$. As shown in this thesis, syndromic surveillance can augment traditional surveillance with timely information on various kinds of health threats. It especially provides also information on the health impact of unexpected and noninfectious threats for which no other surveillance system can provide information. This way, syndromic surveillance can support countries to meet the core surveillance capacity requirements of the IHR (2005).

In the framework of this thesis and the two European Health Programme actions SIDARTHa and Triple S-AGE, a range of concepts and tools have been developed. These can support syndromic surveillance operators in Europe (and beyond) to set up or further develop state-of-the-art syndromic surveillance systems. The syndromic surveillance definition presented in chapter 2 and the generic syndromic surveillance system concept presented in chapters 3 and 5 provide the basis. The guidelines developed as part of the Triple S-AGE project provide developers with a detailed step-by-step handbook on setting up or improving a syndromic surveillance system (23). And, the success factors identified in chapter 6 of this thesis provide operators and decision makers with a priority list of factors to focus on when designing a syndromic surveillance system.

For the European policy level, the Triple S-AGE action has proposed a syndromic surveillance strategy proposing tree implementation models (24). The first model describes a fully decentralised landscape of syndromic surveillance without any connection across borders. This represents the current status of morbidity syndromic surveillance systems. The second model foresees a decentralised data collection but harmonised reporting of findings. This is also what the SIDARTHa concept aims at (chapters 3, 5). And the third model describes a single European syndromic surveillance system. This can only be established for either easily standardised data sources or syndromes such as for mortality, for example (43). The strategy suggests that the three models can be implemented in parallel (24). The Triple S-AGE definition, guidelines and strategy as well as the SIDARTHa system concept provide the framework to set up a locally adjusted but Europe-wide comparable network of syndromic surveillance systems. The concepts are respecting the EU principles of subsidiarity and mutual recognition. At the same time, they foster a certain degree of harmonisation to allow for cross-country comparison of syndromic surveillance output.

The question remains if new knowledge such as generated now for syndromic surveillance actually finds its way into policy and practice. In 2009, the first phase of the Health Programme was evaluated by the European Court of Auditors. They found 'no demonstrable take-up of project results' to a next stage of the research-developmentimplementation cycle and national policy makers not being aware of projects or produced results (44, p.41). Two following evaluations of the last two Public Health Programmes 2003-13 supported these findings. They stated that little dissemination and translation of 
results into policies 'seriously limit the impact of the Health Programme' (45, 46, p.10). To date, the European syndromic surveillance system concept is implemented and active in two regions. Only the future can show if the guidelines produced by the Triple S-AGE action or the key success factors derived from the Qualitative Comparative Analysis are actually picked-up by syndromic surveillance operators. The Triple S-AGE consortium mainly consisted of national institutions responsible for public health surveillance and operators of syndromic surveillance systems. Maybe the chances are good that the concepts are used and spread via these actors.

There is also a lot we do not yet understand about the pick-up of new knowledge in policy and practice. In recent years, the field of knowledge translation research evolved with the aim to enhance the use of evidence in policy and practice (47-49). Tools were developed to enhance knowledge translation targeting policy makers (50) and researchers (51). However, the usefulness and impact of such tools still needs to be evaluated. Furthermore, there is still a lack of understanding about the actual process of the use of new knowledge by policy makers and professionals (52). Future research has also to establish under which circumstances knowledge transfer from one country to another is accomplished and which factors influence transfer and implementation (53).

In order for new knowledge or approaches to be picked-up by policy and practice, it might also be relevant to have high-level policy endorsement. For syndromic surveillance, the WHO assessed the approach in 2001 as not applicable for a global regulation to support countries to meet the IHR (2005) (54). Based on the evidence on the usefulness of syndromic surveillance as presented in this thesis, it might be time for a reassessment. An expansion of syndromic surveillance can position European countries to timely assess the public health impact of potential public health emergencies of international concern, especially rare and non-communicable events. However, in order to achieve a wider rollout of syndromic surveillance in Europe, political endorsement by the national and European levels might be necessary (55).

\section{Conclusions}

Syndromic surveillance can support Europe to timely assess various infectious and noninfectious health threats. In this way, the approach can help European countries to meet the new IHR (2005) surveillance requirements. The thesis presented a syndromic surveillance system concept that fits into the European context by balancing harmonisation efforts and diversity. The thesis also identified key success factors to inform the design of syndromic surveillance systems.

The relatively new approach of syndromic surveillance is still contested. Before a broader implementation could be achieved, more evidence about the usefulness of the approach is necessary - beyond single cases reports. Such evidence was provided in this thesis based on comparisons of multiple syndromic surveillance systems and across countries. The unique European 'laboratory' provided the opportunity to accomplish such comparative research and to generate new knowledge for action. 


\section{References}

1. Buehler JW, Hopkins RS, Overhage JM, Sosin DM, Tong V. Framework for evaluating public health surveillance systems for early detection of outbreaks: recommendations from the CDC Working Group. MMWR Recomm Rep. 2004;53(RR-5):1-11.

2. ScienceDirect. Assessment of syndromic surveillance in Europe. Citing articles [Internet]; 2015 [cited 22 July 2015]. Available from: http://www.sciencedirect.com/science/article/pii/S0140673611608349.

3. Dailey $L$, Watkins RE, Plant AJ. Timeliness of data sources used for influenza surveillance. J Am Med Inform Assoc. 2007;14(5):626-31.

4. Paterson BJ, Durrheim DN. The remarkable adaptability of syndromic surveillance to meet public health needs. J Epidemiol Glob Health.2013;3(1):41-7.

5. Robert Koch Institut. Final presentation and evaluation of epidemiological findings in the EHEC 0104:H4 Outbreak, Germany 2011. Berlin: Robert Koch Institut; 2011.

6. Andersson T, Bjelkmar P, Hulth A, Lindh J, Stenmark S, Widerstrom M. Syndromic surveillance for local outbreak detection and awareness: evaluating outbreak signals of acute gastroenteritis in telephone triage, web-based queries and over-the-counter pharmacy sales. Epidemiol Infect. 2014;142(2):303-13.

7. Loveridge P, Cooper D, Elliot AJ, Harris J, Gray J, Large S, et al. Vomiting calls to NHS Direct provide an early warning of norovirus outbreaks in hospitals. J Hosp Infect. $2010 ; 74(4): 385-93$.

8. Balter S, Weiss D, Hanson H, Reddy V, Das D, Heffernan R. Three years of emergency department gastrointestinal syndromic surveillance in New York City: what have we found? MMWR Morb Mortal Wkly Rep. 2005;54 Suppl:175-80.

9. Rosenkötter N, Ziemann A, Riesgo LG, Gillet JB, Vergeiner G, Krafft T, et al. Validity and timeliness of syndromic influenza surveillance during the autumn/winter wave of A (H1N1) influenza 2009: results of emergency medical dispatch, ambulance and emergency department data from three European regions. BMC Public Health. 2013;13:905. doi: 10.1186/1471-2458-13-905.

10. Rosenkötter N, Ziemann A, Krafft T, Riesgo LG, Vergeiner G, Brand H. Non-infectious events under the International Health Regulations (2005) in Europe - a case for syndromic surveillance. J Public Health Policy. 2014;35(3):311-26. doi: 10.1057/jphp. 2014.

11. Mackenbach J, McKee M, editors. Successes and failures of health policy in Europe Four decades of divergent trends and converging challenges. Maidenhead: Open University Press; 2013.

12. Blackman T, Wistow J, Byrne D. A Qualitative Comparative Analysis of factors associated with trends in narrowing health inequalities in England. Soc Sci Med. 2011;72(12):1965-74.

13. Warren J, Wistow J, Bambra C. Applying qualitative comparative analysis (QCA) in public health: a case study of a health improvement service for long-term incapacity benefit recipients. J Public Health (Oxf). 2014;36(1):126-33. 
14. Kuruvilla S, Schweitzer J, Bishai D, Chowdhury S, Caramani D, Frost L, et al. Success factors for reducing maternal and child mortality. Bull World Health Organ. 2014;92(7):533-44.

15. Glatman-Freedman A, Cohen ML, Nichols KA, Porges RF, Saludes IR, Steffens K, et al. Factors affecting the introduction of new vaccines to poor nations: a comparative study of the Haemophilus influenzae type B and hepatitis B vaccines. PLoS One. 2010;5(11):e13802. doi: 10.1371/journal.pone.0013802.

16. Svevo-Cianci KA, Hart SN, Rubinson C. Protecting children from violence and maltreatment: a qualitative comparative analysis assessing the implementation of U.N. CRC Article 19. Child abuse \& neglect. 2010;34(1):45-56.

17. Brucker DL. Social construction of disability and substance abuse within public disability benefit systems. Int J Drug Policy. 2009;20(5):418-23.

18. Ozegowski S. Effective policy mechanisms for an equitable geographical distribution of general practitioners: a qualitative comparative analysis of the accessibility of primary care in Europe. J Health Serv Res Policy. 2013;18(3):151-9.

19. Melinder KA, Andersson R. The impact of structural factors on the injury rate in different European countries. Eur J Public Health. 2001;11(3):301-8.

20. Conti S, Kanieff M, Rago G, on behalf of the Triple-S project. Inventory of Syndromic Surveillance Systems in Europe; 2012 [cited 26 June 2015]. Available from: http://www.syndromicsurveillance.eu/images/stories/Final_material/tripleS_country_visits.pdf.

21. Ziemann A, Krafft T, Sala Soler M, Sypniewska P. Country visits; 2013 [cited 26 June 2015]. Available from: http://syndromicsurveillance.eu/images/stories/ Final_material/triple-S_country_visits.pdf

22. Dupuy C, Bronner A, Watson E, Wuyckhuise-Sjouke L, Reist M, Fouillet A, et al. Inventory of veterinary syndromic surveillance initiatives in Europe (Triple-S project): current situation and perspectives. Prev Vet Med. 2013;111(3-4):220-9.

23. Triple S Project. Guidelines for designing and implementing a syndromic surveillance system. Triple S Project; 2013 [cited 26 June 2015]. Available from: http://www.syndromicsurveillance.eu /Triple-S_guidelines.pdf

24. Medina S, Fouillet A, Ziemann A, Krafft T, Cooper D, Dupuy C, et al. Proposal for a European strategy for syndromic surveillance. Toward comparability of reporting from syndromic surveillance systems in Europe; 2013 [cited 26 June 2015]. Available from: http://syndromicsurveillance.eu/Triple-S_proposal.pdf.

25. Rosenkötter N, Ziemann A, Riesgo LG, Gillet JB, Vergeiner G, Krafft T, et al. Validity and timeliness of syndromic influenza surveillance during the autumn/winter wave of $A(H 1 N 1)$ influenza 2009: results of emergency medical dispatch, ambulance and emergency department data from three European regions. BMC Public Health. 2013;13(1):905. doi: 10.1186/1471-2458-13-905.

26. Rosenkötter N, Ziemann A, Garcia-Castrillo Riesgo L, Vergeiner G, Fischer M, Krafft T, et al. SIDARTHa Volcanic Ash Cloud Rapid Public Health Impact Assessment. Regional public health impact of volcanic ash cloud covering Europe after eruption of Eyjafjallajoekull, Iceland starting April 14th, 2010. Results as of May 15th, 2010. Bad Honnef: SIDARTHa Scientific-Technical Coordination Office; 2010 [cited 28 June 
2015]. Available from: http://ec.europa.eu/chafea/documents/ health/SIDARTHa_rapid_assessment_volcanic_ash_cloud_15MAY2010.pdf.

27. Greer SL, Fahy N, Elliott HA, Wismar M, Jarman H, Palm W. Everything you always wanted to know about European Union health policies but were afraid to ask. London: World Health Organization European Observatory on Health Systems and Policies; 2014.

28. Ansaldi F, Orsi A, Altomonte F, Bertone G, Parodi V, Carloni R, et al. Syndrome surveillance and molecular epidemiology for early detection and tracing of an outbreak of measles in Liguria, Italy. J Med Virol. 2009;81(10):1807-13.

29. Friedman LS. Real-time surveillance of illicit drug overdoses using poison center data. Clin Toxicol (Phila). 2009;47(6):573-9.

30. May L, Chretien JP, Pavlin JA. Beyond traditional surveillance: applying syndromic surveillance to developing settings--opportunities and challenges. BMC Public Health. 2009;9:242. doi: 10.1186/1471-2458-9-242.

31. Kirkwood A, Guenther E, Fleischauer AT, Gunn J, Hutwagner L, Barry MA. Direct cost associated with the development and implementation of a local syndromic surveillance system. J Public Health Manag Pract. 2007;13(2):194-9.

32. Doroshenko A, Cooper D, Smith G, Gerard E, Chinemana F, Verlander N, et al. Evaluation of syndromic surveillance based on National Health Service Direct derived data--England and Wales. MMWR Morb Mortal Wkly Rep. 2005;54 Suppl:117-22.

33. Jefferson H, Dupuy B, Chaudet H, Texier G, Green A, Barnish G, et al. Evaluation of a syndromic surveillance for the early detection of outbreaks among military personnel in a tropical country. J Public Health (Oxf). 2008;30(4):375-83.

34. Flamand C, Larrieu S, Couvy F, Jouves B, Josseran L, Filleul L. Validation of a syndromic surveillance system using a general practitioner house calls network, Bordeaux, France. Euro Surveill. 2008;13(25). pii: 18905.

35. Ijaz K, Kasowski $\mathrm{E}$, Arthur RR, Angulo FJ, Dowell SF. International Health Regulations-What Gets Measured Gets Done. Emerg Infec Dis. 2012;18(7):1054-7.

36. Schenkel K, Williams C, Eckmanns T, Poggensee G, Benzler J, Josephsen J, et al. Enhanced surveillance of infectious diseases : the 2006 FIFA World Cup experience, Germany. Euro Surveill. 2006;11(12):234-8.

37. Wadl M, Rieck T, Nachtnebel M, Greutelaers B, an der Heiden M, Altmann D, et al. Enhanced surveillance during a large outbreak of bloody diarrhoea and haemolytic uraemic syndrome caused by Shiga toxin/verotoxin-producing Escherichia coli in Germany, May to June 2011. Euro Surveill. 2011;16(24). pii: 19893.

38. Groenewegen PP. Analyzing European health systems: Europe as a research laboratory. Eur J Public Health. 2013;23(2):185-6.

39. Karanikolos M, Mladovsky P, Cylus J, Thomson S, Basu S, Stuckler D, et al. Financial crisis, austerity, and health in Europe. The Lancet. 2013;381(9874):1323-31.

40. European Parliament and Council of the European Union. Decision on serious crossborder threats to health and repealing Decision No 2119/98/EC (1082/2013/EU). Off J Eur Union. 2013;L 293:1-15. 
41. Kaydos-Daniels SC, Rojas Smith L, Farris TR. Biosurveillance in outbreak investigations. Biosecur Bioterror. 2013;11(1):20-8.

42. Koopmans M. Surveillance strategy for early detection of unusual infectious disease events. Curr Opin Virol. 2013;3(2):185-91.

43. EuroMOMO [Internet]; 2013 [cited 26 June 2015]. Available from: http://www.euromomo.eu.

44. European Court of Auditors. The European Union's Public Health Programme (200307). An effective way to improve health? Special Report 2/2009. Luxembourg: European Communities; 2009.

45. COWI. DG SANCO Ex-post evaluation of the Public Health Programme (PHP) 20032008. Final Report. Kongens Lyngby; 2011.

46. Public Health Evaluation and Impact Assessment Consortium. Specific contract: MidTerm Evaluation of the Health Programme (2008-2013). Final Report; 2011.

47. Graham I, Logan J, Harrison M, Straus S, Tetro J, Caswell W. Lost in knowledge translation: Time for a map? J Contin Educ Health Prof. 2006;26:13-24.

48. Moat KA, Lavis JN. 10 best resources for ... evidence-informed health policy making. Health Policy Plan. 2013;28(2):215-8.

49. Lomas J. The in-between world of knowledge brokering. BMJ. 2007;334:129-32.

50. Lavis J, Oxman A, Lewin S, Fretheim A. SUPPORT Tools for evidence-informed health Policymaking (STP). BMC Health Res Policy Syst. 2009;7(Suppl 1):I1. doi:10.1186/1478-4505-7-S1-I1.

51. BRIDGE series [Internet]; 2015 [cited 26 June 2015]. Available from: http://www.euro.who.int/en/about-us/partners/observatory/bridge-series.

52. Oliver K, Lorenc T, Innvaer S. New directions in evidence-based policy research: a critical analysis of the literature. BMC Health Res Policy Syst 2014;12:34. doi:10.1186/1478-4505-12-34

53. Hansen J, de Jong J, Groenewegen P, Ricciardi W. Building sustainable and resilient health care systems: How ERA-NETs in Horizon 2020 can help. Eurohealth. 2015;21(1):28-31.

54. Revision of the International Health Regulations. Progress report, February 2001. Wkly Epidemiol Rec. 2001;8:61-3.

55. Ziemann A, Rosenkötter N, Garcia-Castrillo Riesgo L, Fischer M, Krämer A, Lippert FK, et al. Meeting the International Health Regulations (2005) surveillance core capacity requirements at the subnational level in Europe: the added value of syndromic surveillance. BMC Public Health. 2015;15:107. doi:10.1186/s12889-0151421-2. 

Valorisation 


\section{Relevance of research results}

The innovative aspect of the research results is the provision of new knowledge on the application of syndromic surveillance in Europe. There is doubt about the usefulness of syndromic surveillance among public health professionals and decision makers $(1,2)$. The research results add clarity on the strengths and weaknesses of the approach and its applicability and usefulness in the European context. The research was accomplished based on the work of the two Health Programme actions SIDARTHa and Triple S-AGE and as such was from the beginning oriented towards relevance for practice and decision making in public health surveillance. The analysis of actually applied syndromic surveillance systems or as part of case studies support the practical relevance of the results. Especially, the new definition, the generic syndromic surveillance system concept, and the success factors provide concrete input for public health surveillance. The SIDARTHa system concept was implemented at the regional level in Austria and Spain. During the Triple S-AGE action, some of the research results regarding the SIDARTHa project were included in the European guidelines for syndromic surveillance systems (3). These form a handbook for public health authorities who intend to implement or improve syndromic surveillance systems and are available from the Triple S-AGE website (4).

There are three valorisation areas for which the research results are relevant beyond science:

1. the improvement of the surveillance and early warning capacity of public health authorities,

2. the potential to enhance general public health monitoring based on syndromic data sources, and

3. the support of timely management of resources in health services.

Figure 1 provides an overview of the different aspects of the valorisation process for each area that is explained in more detail in the following.

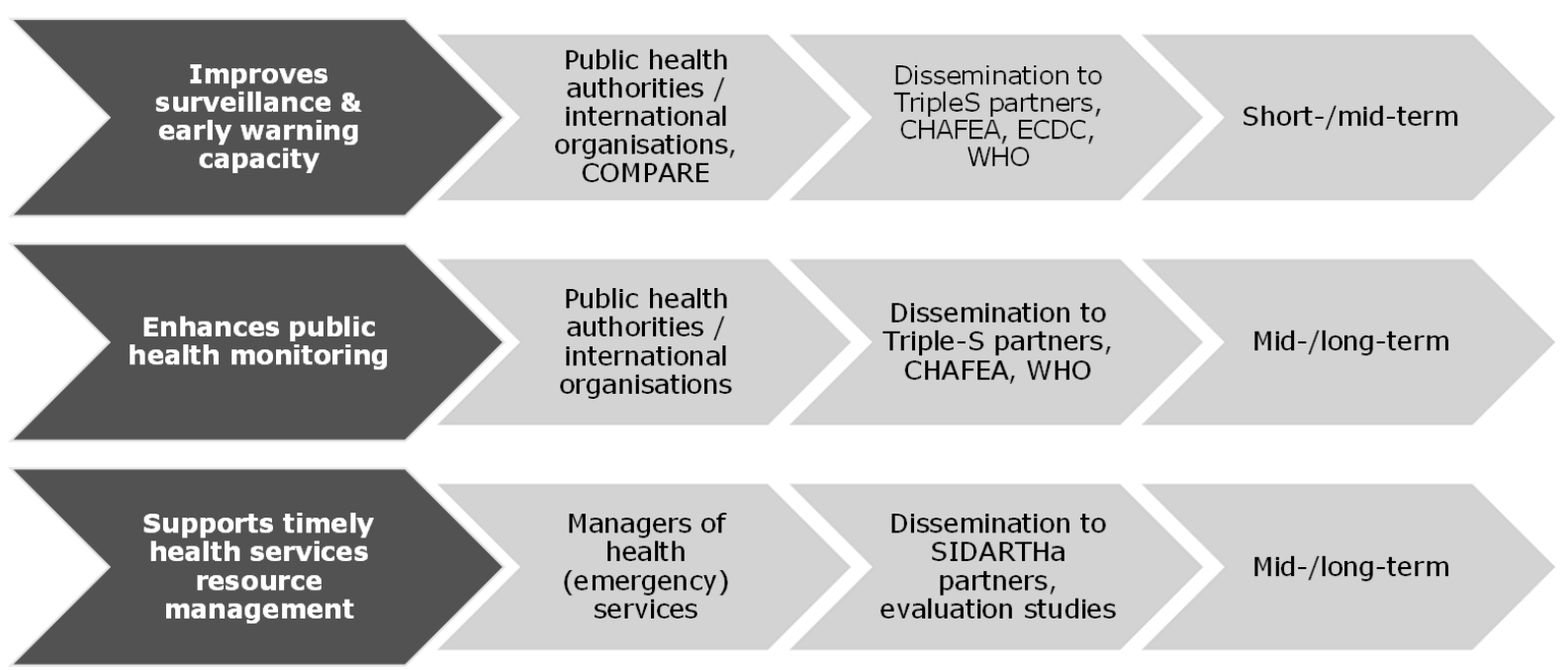

Figure 1: Main aspects of the valorisation process

CHAFEA = Consumers, Health, Agriculture and Food Executive Agency (European Commission); ECDC = European Centre for Disease Prevention and Control; WHO = World Health Organization (Regional Office for Europe) 


\section{Improves surveillance and early warning capacity}

The research provides additional knowledge relevant for improving the surveillance capacity of public health authorities. The new syndromic surveillance definition, system concept and success factors can support syndromic surveillance operators to set up or improve syndromic surveillance systems. An increased and improved use of syndromic surveillance can enhance the ability of public health authorities at local, regional, and national level to timelier assess expected or emerging health threats. The exploitation of existing data sources for public health surveillance through syndromic surveillance adds an additional piece to the health information puzzle that can help identify and track health threats.

First concrete valorisation activities in this area would focus on the dissemination of the research results. The main target group would be public health authorities and European organisations that can influence the transfer of knowledge and innovations in this area. As the research was based on the work of the Triple S-AGE action, the partner organisations involved in the action, mainly national public authorities, would be the primary target group for disseminating the research results. The partners could use the research results to improve their own syndromic surveillance systems. Further, the European Centre for Disease Prevention and Control, the European Commission's Health Threat Unit and the World Health Organization Regional Office for Europe were part of the project. Awareness about the research results among these institutions could help to further disseminate the research results. We will send a copy of this thesis in 2015 to all project partners and the European Commission's Consumers, Health, Agriculture and Food Executive Agency (CHAFEA) that handled the Triple S-AGE action. We think it very likely that those Triple S-AGE partners who are operating syndromic surveillance systems will use the research results in short to mid-term.

Furthermore, there is a new European research project called COMPARE, co-funded by the European Commission as part of the Horizon 2020 programme (5). The COMPARE project intends to integrate different information sources for a timelier detection of and response to disease outbreaks among humans and animals. The author of this thesis and the supervisor Thomas Krafft are involved as advisors on syndromic surveillance in the project. To raise awareness on the research results, we will send the thesis also to the co-coordinator of the project, Marion Koopmans. The project just started and there is a chance that the research results inform the project activities in the next couple of years.

Finally, the e-book version of this thesis could be included on the web-platform set up by the Triple S-AGE action, which can be seen as the primary information source on syndromic surveillance in Europe (4). Furthermore, it could be included also on the primary web-platform worldwide, hosted by the International Society for Disease Surveillance, that was involved as advisor in the Triple S-AGE action (6). Also the CHAFEA could be asked to include the thesis or a link to the thesis on their website. We will approach the three organisations in 2015. It is likely that public health authorities who intend to setup a syndromic surveillance system in the next couple of years are visiting these web-platforms and might include the research results in their work. 


\section{Enhances public health monitoring}

The research has shown the use of syndromic information to monitor various health threats, especially also non-communicable threats. This yields the potential of syndromic data sources and indicators to be used also for general public health monitoring. Some of the partner organisations of the SIDARTHa action, including the author and the supervisor Thomas Krafft, were involved in another Health Programme Action, the "European Emergency Data Project" (EED Project). The EED Project partners defined and tested key health monitoring indicators based on routine emergency care data - the same data source used in the SIDARTHa action for syndromic surveillance. They have defined five key indicators, which were included in the European Community Health Indicators long list $(7,8)$. The author and the supervisor Thomas Krafft were also involved in a case study in Germany on the added value and feasibility of pre-hospital emergency care data for the European Injury Data Base (9). The study showed that many indicators, which are required for the Injury Data Base were retrievable from pre-hospital emergency care data and even provided additional data compared to the usually registered data from hospitals. Recently, the World Health Organization has acknowledged the potential of non-traditional data sources such as electronic patient records for public health monitoring in their 2015 European Health Report (10).

The focus of the valorisation activities in this area is also on disseminating the research results. As part of the dissemination activities described above, we will inform the same target groups about the potential of the syndromic data sources for other areas of public health surveillance and public health monitoring. We will ask our contacts at the organisations to send the link to the e-book version of the thesis to the colleagues in their organisations who are responsible for public health monitoring. We will also disseminate the e-book version to the Division of Information, Evidence, Research and Innovation of the WHO Regional Office for Europe, who are the editors of the European Health Report, and to which the supervisor Helmut Brand serves as advisor. The research results can provide awareness about the approach and its potential use for health monitoring but further research is warranted to build a sound evidence base. Therefore, the valorisation of the research results in this area is rather indirect and mid to long-term.

\section{Enables timely health services resource management}

Syndromic surveillance information might also be useful for resource management in health services. As indicated in chapter 4 , the SIDARTHa syndromic surveillance system that is implemented based on emergency department data in Spain was the only source of timely information on the peak of the influenza season during the Christmas holidays in one season. The head of the emergency department used the information provided by the syndromic surveillance system in that season to call further staff members into the emergency department to cope with the increasing number of patients. The SIDARTHa syndromic surveillance systems are intended to be implemented in the institutions that are providing data such as emergency service providers, not in the public health authorities. This allows for better awareness of and direct access of managers in these institutions to the syndromic surveillance information.

Also for this area, the focus of the valorisation activities is on dissemination. The major target group are the partners of the SIDARTHa action, which were mainly representing regional-level emergency care institutions from different countries. We will send the 
thesis to all SIDARTHa partners and the CHAFEA, with an enclosed letter asking for further spread of the link to the e-book-version to other emergency care institutions in their networks. In 2015, the e-book-version will also be included for download on the websites of the SIDARTHa action (11) and the European Emergency Data Research Network (8), combined with a news item posted on the home pages. In the following years, the two active SIDARTHa system implementations in Austria and Spain could be evaluated for this purpose. This could be done as part of placements of Bachelor or Master students of Maastricht University's European Public Health programme, in which both supervisors are involved. We think that the use of the research results in this area will depend on the demonstration of the usefulness in the two implementation sites or other institutions involved in the SIDARTHa action. Therefore, the valorisation of the research results in this area will rather be mid- to long-term. 


\section{References}

1. Koopmans M. Surveillance strategy for early detection of unusual infectious disease events. Curr Opin Virol. 2013;3(2):185-91.

2. Kaydos-Daniels SC, Rojas Smith L, Farris TR. Biosurveillance in outbreak investigations. Biosecur Bioterror. 2013;11(1):20-8.

3. Triple S Project. Guidelines for designing and implementing a syndromic surveillance system; 2013 [cited 29 September 2015]. Available from: http://www.syndromicsurveillance.eu/Triple-S_guidelines.pdf.

4. Triple S-AGE [Internet]; 2015 [cited 29 September 2015]. Available from: www.syndromicsurveillance.eu.

5. COMPARE [Internet]; 2015 [cited 29 September 2015]. Available from: www.compare-europe.eu.

6. International Society for Disease Surveillance [Internet]; 2015 [cited 29 September]. Available from: www.syndromic.org.

7. Krafft T, Garcia Castrillo-Riesgo L, Edwards S, Fischer M, Overton J, RobertsonSteel I, et al. European Emergency Data Project (EED Project): EMS data-based health surveillance system. Eur J Public Health. 2003;13(3 Suppl):85-90

8. European Emergency Data Research Network [Internet]; 2015 [cited 29 September 2015]. Available from: www.eed-network.eu.

9. Krafft T, Ziemann A. Notfallmedizin und öffentliche Gesundheit - Einsatz notfallmedizinischer Daten für Fragen der öffentlichen Gesundheit in Europa In: Neumayr A, Schinnerl A, Baubin M, editors. Qualitätsmanagement in der prähospitalen Notfallmedizin. Heidelberg: Springer; 2013. p. 31-42.

10. World Health Organization Regional Office for Europe. The European Health Report 2015. Targets and beyond - reaching new frontiers in evidence. Copenhagen: World Health Organization; 2015.

11. SIDARTHa [Internet]; 2015 [cited 29 September 2015]. Available from: http://www.sidartha.eu. 


\section{Summary}

Samenvatting

Zusammenfassung 


\section{Summary}

\section{Background}

Our globalised world faces new, unprecedented and diverse challenges for public health from new emerging diseases, natural disasters, or man-made threats. They call for quick decisions and actions, which can only be taken based on rapid information about the public health impact of events. Until now, traditional public health surveillance was focusing on specific pre-defined diseases or agents causing certain diseases. It cannot provide information rapidly or on various kinds of health threats. Syndromic surveillance can fill this gap. It aims at augmenting traditional public health surveillance systems with (near) real-time information on the public health impact of events. Syndromic surveillance analyses existing, possibly electronic data that usually were not collected for surveillance purposes such as from web searches or patient records. The approach is relatively new and there is still much doubt about its added value.

Europe or the European Union is a unique structure, which supports member states who are actually responsible for health issues, to join forces for fighting health threats and to learn from each other to generate new knowledge. Three dimensions of learning can be differentiated, with the European context playing an increasing role from the first to the third dimension. In the first dimension, Europe provides a context in which countries can learn from each other to gain new knowledge for their own situation ('Learning in Europe'). In the second dimension, knowledge for Europe is compiled by harmonisation, standardisation, generalisation or identification of a common denominator ('Learning for Europe'). Here, the European Union principles such as harmonisation, subsidiarity and mutual recognition should be taken into account in order for the knowledge to fit into the European context. The third dimension is focusing on generating new knowledge, based on a structured analysis of the differences between countries ('learning from Europe').

This thesis aimed at analysing (1) the added value of syndromic surveillance for improving Europe's surveillance capacity, and (2) the added value of the European context to gain new knowledge about syndromic surveillance.

\section{Methods}

For the first aim, the thesis first explored how a specific European definition for syndromic surveillance and a syndromic surveillance system should look like by respecting the European principles of harmonisation, mutual recognition and subsidiarity. This was achieved by applying a consensus method approach, which was informed by the results of a semi-structured survey and a literature review. Secondly, the usefulness of syndromic surveillance for Europe was analysed by assessing the major strengths and weakness of syndromic surveillance: timeliness, flexibility, additional information provision, and non-specificity. The analysis focused on three main purposes of syndromic surveillance: surveillance of (seasonal) influenza and gastrointestinal outbreaks, and situational awareness during various events. Finally, the thesis assessed how the application of syndromic surveillance can foster Europe's capacity to meet the new International Health Regulation's (2005) surveillance core capacity requirements. These two analysis steps were based on quantitative data analyses in a case study design, using secondary data from emergency care and a narrative review based on mixed data collected from the literature. For the second aim, the thesis analysed how the European context provides an added value for learning about syndromic surveillance. The focus of 
this analysis was on a cross-country comparison for identifying success factors of syndromic surveillance systems. For this step, the mixed methods approach Qualitative Comparative Analysis was applied, analysing mixed data collected from the literature and from country visits.

\section{Results}

The new syndromic surveillance definition is more comprehensive and generic than the earlier definition by the Centers for Disease Control and Prevention. The syndromic surveillance system concept is balancing the European principals of harmonisation, mutual recognition and subsidiarity. Instead of recommending one harmonised European syndromic surveillance system, the concept suggests to implement a network of subnational systems. The concept performed best if adjusted to local or regional circumstances. The surveillance results were anticipated to still be comparable across systems and borders. The system is implemented in two regions in Europe at the moment, indicating its applicability in the European context.

Syndromic surveillance is useful for Europe in terms of providing timely and additional information on various kinds of health threats. The main strength of syndromic surveillance, timeliness, was confirmed when assessing cases for all three purposes. The strength of flexibility was confirmed for situational awareness during diverse events such as the 2009 influenza pandemic or the influx of migrants from North Africa to Italy in 2011. Syndromic surveillance is especially useful at times when it can provide additional information on the public health impact of an event or on population groups that are not monitored by traditional surveillance. Non-specificity applies to certain data sources, syndromes or events but is not a general weakness of syndromic surveillance. Further, syndromic surveillance can support the implementation of the core surveillance capacity requirements of the International Health Regulations (2005). It can help detect and assess the local and regional impact of different types of public health emergencies in a timely manner. This might especially hold true for rare and non-infectious health threats for which no (timely) information on the public health impact is available from other systems.

Regarding the added value of the European context for gaining new knowledge about syndromic surveillance, the thesis showed that the new definition and the system concept were generated as a result of 'learning for Europe'. The thesis especially showed that new syndromic surveillance knowledge could be derived by 'learning from Europe', based on the comparative analysis of syndromic surveillance systems from different European countries. Here, key success factors for syndromic surveillance systems were identified which can inform decision makers in Europe and beyond when setting up or further developing syndromic surveillance systems. The analysis showed that timeliness of syndromic influenza surveillance might be increased when non-clinical data sources are analysed. Furthermore, syndromic situational awareness can be enhanced if multiple syndromes are analysed in automated systems.

\section{Conclusions}

Syndromic surveillance can support Europe to timely assess various infectious and noninfectious health threats. In this way, the approach can help European countries to meet 
the new International Health Regulation's (2005) surveillance requirements. The thesis presented a syndromic surveillance system concept that fits into the European context by balancing harmonisation efforts and diversity. The thesis also identified key success factors to inform the design of syndromic surveillance systems.

Before a broader implementation of syndromic surveillance could be achieved, more evidence about the usefulness of the approach was necessary - beyond single cases reports. The thesis provided such evidence based on comparisons of multiple syndromic surveillance systems and across countries. The unique European 'laboratory' provided the opportunity to accomplish such comparative research and to generate new knowledge for action.

\section{Samenvatting}

\section{Achtergrond}

Onze geglobaliseerde wereld wordt geconfronteerd met nieuwe, onbekende en verschillende uitdagingen voor de volksgezondheid door nieuw ontstane ziekten, natuurrampen of door de mens gemaakte gevaren. Deze vragen om snelle beslissingen en acties, die alleen gebaseerd op snelle informatie over de effecten van incidenten op de volksgezondheid kunnen worden genomen. Tot nu toe was traditionele volksgezondheidssurveillance gericht op specifieke, vooraf gedefinieerde ziekten of verwekkers van specifieke ziekten. Informatie is niet snel en ook niet voor verschillende soorten van bedreigingen van de gezondheid beschikbaar. Syndroomsurveillance kan deze lacune opvullen. Het is gericht op het aanvullen van traditionele surveillance met (bijna) realtime informatie over de impact van een incident op de volksgezondheid. Syndroomsurveillance analyseert bestaande, zo mogelijk elektronische data, die gewoonlijk niet voor surveillance reden zijn verzameld, bijvoorbeeld van zoekacties op internet of patientendossiers. Deze aanpak is relatief nieuw en er bestaat nog steeds twijfel over de meerwaarde ervan.

Europa of de Europese Unie is een unieke constructie die hun lidstaten, die eigenlijk verantwoordelijk zijn voor gezondheidszaken, steunen om gezamenlijk gezondheidsgevaren te bestrijden of van elkaar te leren om nieuwe kennis te genereren. Drie dimensies van leren kunnen worden onderscheiden met een toenemende rol van de Europese context van de eerste tot de derde dimensie. In de eerste dimensie voorziet Europa een omgeving waarin staten van elkaar kunnen leren om nieuwe kennis voor hun eigen situatie te verzamelen ('leren in Europa'). In de tweede dimensie wordt kennis voor Europa verzameld door harmonisatie, standaardisatie, generalisatie of identificatie van een gemeenschappelijke basis ('leren voor Europa'). Hier zouden de principes van de Europese Unie zoals harmonisatie, subsidiariteit en wederzijdse erkenning worden gehanteerd zodat daarmee de kennis in de Europese context past. De derde dimensie is gericht op het genereren van nieuwe kennis gebaseerd op een gestructureerde analyse van verschillen tussen landen ('leren van Europa').

Dit proefschrift was gericht op het analyseren van (1) de meerwaarde van syndroomsurveillance voor het verbeteren van de Europese surveillance capaciteit, en (2) de meerwaarde van de Europese context voor het genereren van nieuwe kennis over syndroomsurveillance. 


\section{Methode}

Voor het eerste doel is in het proefschrift ten eerste verkent hoe een specifieke Europese definitie voor syndroomsurveillance en een syndroomsurveillancesysteem eruit kan zien die de Europese principes harmonisatie, wederzijdse erkenning en subsidiariteit respecteren. Dit is bereikt door toepassing van consensusmethoden gebaseerd op de resultaten van een semi-gestructureerde survey en literatuuronderzoek. Ten tweede werd de bruikbaarheid van syndroomsurveillance voor Europa geanalyseerd door beoordeling van de sterkten en zwakte van syndroomsurveillance: actualiteit, flexibiliteit, extra informatie voorziening en niet-specificiteit. De analyse was gefocust op de drie belangrijkste doelen: surveillance van (seizoens) influenza en gastro-enteritis uitbraken, en situationele bewustzijn of situational awareness tijdens diverse incidenten. Ten slotte heeft dit proefschrift beoordeeld hoe het gebruik van syndroomsurveillance de Europese capaciteit kan verbeteren om aan de kernvoorwaarden voor surveillancecapaciteiten van de nieuwe Internationale Gezondheidsregelingen (2005) te voldoen. Deze twee analysestappen waren gebaseerd op kwantitatieve data analyses van routinematig verzamelde data uit de acute zorg in een casestudie opzet en een narratieve analyse van mixed data uit de literatuur. Voor het tweede doel is geanalyseerd hoe de Europese context een meerwaarde toevoegt aan het leren over syndroomsurveillance. De focus van deze analyse lag op een vergelijking van meerdere landen voor het identificeren van succesfactoren van syndroomsurveillancesysteemen. Voor deze stap werd de mixedmethod aanpak Qualitative Comparative Analysis toegepast, waarbij mixed data verzameld uit de literatuur en gegevens door het bezoeken van verschillende landen geanalyseerd werden.

\section{Resultaten}

De nieuwe syndroomsurveillance definitie is uitgebreider en generieker dan de vroegere definitie van de Centers for Disease Control and Prevention. Het syndroomsurveillancesysteem concept balanceert tussen de Europese principes harmonisatie, wederzijdse erkenning en subsidiariteit. In plaats van het aanbevelen van één gestandaardiseerd Europees syndroomsurveillancesysteem stelt het concept de implementatie van een netwerk van regionale systemen voor. Dit concept werkt het best indien het toegepast wordt op lokale of regionale omstandigheden. De verwachting is dat de surveillanceresultaten nog steeds over landsgrenzen en systemen heen vergelijkbaar zijn. Het systeem is op dit moment geïmplementeerd in twee regio's in Europa waardoor de toepasbaarheid van het concept voor de Europese context wordt aangetoond.

Syndroomsurveillance is bruikbaar voor Europa met betrekking tot het aanleveren van actuele en aanvullende informatie voor diverse soorten van gezondheidsgevaren. Het belangrijkste sterke punt van syndroomsurveillance, actualiteit, werd bevestigd bij het onderzoeken van situaties voor alle drie doeleinden. Het sterke punt flexibiliteit werd bevestigd voor situational awareness tijdens diverse incidenten zoals bijvoorbeeld de influenza pandemie in 2009 of de instroming van immigranten uit Noordafrika naar Italie in 2011. Syndroomsurveillance is van bijzondere meerwaarde indien het extra informatie kan leveren over de impact van een incident of over specifieke populaties die niet worden gemonitord door traditionele surveillance. Niet-specificiteit geldt voor specifieke bronnen van data, syndromen of incidenten maar is geen algemeen zwak punt van syndroomsurveillance. Verder kan syndroomsurveillance de implementatie van de kernvoorwaarden voor surveillancecapaciteit van de Internationale 
Gezondheidsregelingen (2005) steunen. Het kan het detecteren en beoordelen van lokale en regionale effecten van verschillende soorten van volksgezondheidsgevaren op een actuele manier ondersteunen. Dit geldt vooral voor ongewone en niet-besmettelijke gevaren waarvoor (actuele) informatie over de impact op de volksgezondheid op basis van andere systemen niet beschikbaar is.

Met betrekking tot de meerwaarde van de Europese context voor het genereren van nieuwe kennis over syndroomsurveillance heeft dit proefschrift laten zien dat de nieuwe definitie en het systeemconcept als resultaat van 'leren voor Europa' zijn ontstaan. Dit proefschrift heeft vooral aangetoond dat nieuwe kennis over syndroomsurveillance werd genereerd door 'leren van Europa', gebaseerd op de vergelijkende analyse van syndroomsurveillancesystemen in verschillende landen. Hier werden sleutelsuccesfactoren voor syndroomsurveillancesystemen geïdentificeerd die besluitnemers in Europa en daaroverheen kunnen gebruiken indien deze een syndroomsurveillancesysteem willen inrichten of doorontwikkelen. De analyse heeft laten zien dat de actualiteit van syndroomsurveillance voor influenza geoptimaliseerd kan worden door het analyseren van niet-klinische data bronnen. Verder kan de situational awareness verbeterd worden door het analyseren van meerdere syndromen in geautomatiseerde systemen.

\section{Conclusie}

Syndroomsurveillance kan Europa steunen door een actuele beoordeling van verschillende besmettelijke en niet-besmettelijke gezondheidsgevaren. Daardoor kan deze aanpak Europese landen helpen aan de surveillance voorwaarden van de nieuwe Internationale Gezondheidsregelingen (2005) te voldoen. Dit proefschrift heeft een concept voor een syndroomsurveillancesysteem gepresenteerd dat in de Europese context past door het balanceren tussen het streven naar harmonisatie en diversiteit. Het proefschrift heeft ook sleutelsuccesfactoren geïdentificeerd die gebruikt kunnen worden in het design van syndroomsurveillancesystemen.

Voordat een bredere implementatie van syndroomsurveillance kan worden bereikt was meer bewijs nodig over de meerwaarde van de aanpak - over enige casestudies heen. Dit proefschrift heeft dit bewijs geleverd gebaseerd op het vergelijken van meerdere syndroomsurveillancesystemen en meerdere landen. Het unieke Europese 'laboratorium' heeft de mogelijkheid voor dit soort vergelijkend onderzoek geboden en heeft nieuwe kennis voor vervolgactiviteiten opgeleverd.

\section{Zusammenfassung}

\section{Hintergrund}

Unsere globalisierte Welt steht vor neuen, unbekannten und vielschichtigen Herausforderungen für die öffentliche Gesundheit, durch neu auftretende Krankheiten, Naturkatastrophen oder von Menschenhand geschaffene Gefahren. Diese fordern schnelle Entscheidungen und Maßnahmen, die nur basierend auf schnell verfügbaren Informationen zu den Auswirkungen der Ereignisse auf die Gesundheit der Bevölkerung getroffen werden können. Bisher war die traditionelle Gesundheitsüberwachung oder Surveillance auf spezielle vordefinierte Krankheiten oder Krankheitserreger gerichtet. Diese kann Informationen weder schnell noch $\mathrm{zu}$ verschiedenen Arten von 
Gesundheitsgefahren bereitstellen. Syndromsurveillance kann diese Lücke füllen. Sie hat das Ziel, die traditionelle Surveillance mit Informationen zur Auswirkung von diversen Gesundheitsgefahren auf die öffentliche Gesundheit (beinahe) in Echtzeit anzureichern. Syndromsurveillance analysiert bestehende, möglichst elektronische Daten, die gewöhnlich nicht für Surveillancezwecke gesammelt wurden, wie beispielsweise von Internetsuchanfragen oder aus Patientenakten. Der Ansatz ist relativ neu und es besteht noch Zweifel über seinen Mehrwert.

Europa bzw. die Europäische Union ist ein einzigartiges Konstrukt, das die Mitgliedstaaten, die eigentlich für Gesundheitsfragen verantwortlich sind, dabei unterstützt, ihre Kräfte für die Bekämpfung von Gesundheitsgefahren zu bündeln oder voneinander zu lernen, um neues Wissen zu generieren. Es lassen sich drei Dimensionen des Lernens unterscheiden, wobei der europäische Kontext von der ersten bis zur dritten Dimension eine zunehmende Rolle spielt. In der ersten Dimension bietet Europa eine Umgebung, in der Länder voneinander lernen, um neues Wissen für ihre eigene Situation zu kreieren (,Lernen in Europa'). In der zweiten Dimension wird Wissen für Europa zusammengetragen durch Harmonisierung, Standardisierung, Generalisierung oder Identifikation eines gemeinsamen Nenners (,Lernen für Europa'). Hier sollten die europäischen Prinzipien wie Harmonisierung, Subsidiarität und gegenseitige Anerkennung mitbetrachtet werden, damit das Wissen in den europäischen Kontext passt. Die dritte Dimension konzentriert sich auf das Generieren neuen Wissens basierend auf strukturierten Analysen der Unterschiede zwischen Ländern (,Lernen von Europa').

Diese Dissertation hatte zum Ziel (1.) den Mehrwert von Syndromsurveillance für die Verbesserung von Europas Surveillancekapazität und (2.) den Mehrwert des europäischen Kontextes zur Generierung neuen Wissens über Syndromsurveillance zu untersuchen.

\section{Methoden}

Für das erste Ziel wurde in der Dissertation zunächst untersucht wie eine spezifisch europäische Definition für Syndromsurveillance und ein spezifisch europäisches Syndromsurveillancesystem aussehen könnten, indem die europäischen Prinzipien Harmonisierung, gegenseitige Anerkennung und Subsidiarität berücksichtigt werden. Dies wurde erreicht durch das Anwenden eines Konsensusmethodenansatzes und diesem zugrunde liegenden Ergebnissen einer halbstandardisierten Befragung und einer Literaturanalyse. Zweitens wurde der Nutzen von Syndromsurveillance für Europa durch die Bewertung ihrer hauptsächlichen Stärken und Schwäche analysiert: Aktualität, Flexibilität, Bereitstellung zusätzlicher Informationen und nicht-Spezifität. Die Analyse konzentrierte sich auf die drei Hauptanwendungsgebiete von Syndromsurveillance: Surveillance von (saisonalen) Influenza- und Gastroenteritisausbrüchen, und Lagebewußtsein oder Situational Awareness während unterschiedlicher Ereignisse. Zuletzt wurde in der Dissertation untersucht wie die Anwendung von Syndromsurveillance Europa darin unterstützen kann, die neuen Anforderungen für Surveillancekernkapazitäten der Internationalen Gesundheitsvorschriften (2005) einzuhalten. Diese zwei Analyseschritte basierten auf der quantitativen Analyse von routinemäßig erhobenen Daten der Notfallversorgung und einem narrativen Review von gemischten Daten aus der Literatur. Für das zweite Ziel wurde in der Dissertation untersucht wie der europäische Kontext einen Mehrwert für das Lernen über Syndromsurveillance liefert. Der Fokus dieser Analyse lag auf einem Ländervergleich zur Identifikation von Schlüsselerfolgsfaktoren für Syndromsurveillancesysteme. Für diesen 
Schritt ist die gemischte Methode Qualitative Comparative Analysis zur Anwendung gekommen, mit der gemischte Daten aus der Literatur und von Standortbesichtigungen in verschiedenen Ländern untersucht wurden.

\section{Ergebnisse}

Die neue Syndromsurveillancedefinition ist umfassender und allgemeiner anwendbar als die frühere Definition der Centers for Disease Control and Prevention. Das Syndromsurveillancesystemkonzept findet einen Ausgleich zwischen den europäischen Prinzipien Harmonisierung, gegenseitige Anerkennung und Subsidiarität. Statt ein harmonisiertes europäisches Syndromsurveillancesystem zu empfehlen, sieht das Konzept die Implementierung eines Netzwerks regionaler Systeme vor. Das Konzept funktionierte am besten, wenn es an die lokalen oder regionalen Umstände angepasst wurde. Die Surveillanceergebnisse sind voraussichtlich dennoch über Systeme und Landesgrenzen hin vergleichbar. Das System ist momentan in zwei Regionen in Europa implementiert, wodurch die Anwendbarkeit im europäischen Kontext demonstriert wird.

Syndromsurveillance ist für Europa im Hinblick auf die Bereitstellung von kurzfristigen und zusätzlichen Informationen zu verschiedenen Gesundheitsgefahren von Nutzen. Die hauptsächliche Stärke von Syndromsurveillancesystemen, Aktualität, wurde in der Untersuchung von Fällen aus allen drei Hauptanwendungsgebieten bestätigt. Die Stärke Flexibilität wurde für Situational Awareness während verschiedener Ereignisse, wie beispielsweise die Influenzapandemie 2009 oder die Einreisewelle von Flüchtlingen aus Nordafrika 2011 bestätigt. Syndromsurveillance ist besonders nützlich, wenn es zusätzliche Informationen zu den Auswirkungen auf die öffentliche Gesundheit oder auf bestimmte Bevölkerungsgruppen liefern kann, die nicht durch traditionelle Surveillancesysteme überwacht werden. Nicht-Spezifität kommt nur für bestimmte Datenquellen, Syndrome oder Ereignisse zum Tragen, ist aber keine generelle Schwäche von Syndromsurveillance. Des Weiteren kann Syndromsurveillance die Umsetzung der Kernanforderungen zur Surveillancekapazität der Internationalen Gesundheitsvorschriften (2005) erleichtern. Sie kann bei der schnelleren Entdeckung und Bewertung von lokalen und regionalen Auswirkungen verschiedener Arten von Gefahren für die öffentliche Gesundheit Unterstützung leisten. Dies gilt vor allem für seltene und nicht-infektiöse Gesundheitsgefahren, für die keine aktuellen Informationen zu den Auswirkungen auf die öffentliche Gesundheit durch andere Systeme vorliegen.

Bezüglich des Mehrwerts des europäischen Kontextes für die Erwerbung neuen Wissens über Syndromsurveillance hat die Dissertation gezeigt, dass die neue Definition und das Systemkonzept aus einem ,Lernen für Europa' heraus generiert wurden. Die Dissertation hat insbesondere zeigen können, dass basierend auf der vergleichenden Analyse von Syndromsurveillancesystemen verschiedener Länder neues Wissen durch ,Lernen von Europa' abgeleitet werden konnte. Dabei sind Schlüsselerfolgsfaktoren für Syndromsurveillancesysteme identifiziert worden, die Entscheidungsträger in Europa und darüber hinaus bei der Implementierung oder Weiterentwicklung von Syndromsurveillancesystemen berücksichtigen können. Die Analyse hat ergeben, dass die Aktualität von syndromischer Influenzasurveillance durch die Analyse nicht-klinischer Daten gesteigert werden könnte. Des Weiteren kann Situational Awareness durch die Analyse mehrerer Syndrome in automatisierten Systemen verbessert werden. 


\section{Schlussfolgerungen}

Syndromsurveillance kann Europa dabei unterstützen, in kurzer Zeit verschiedenste infektiöse und nicht-infektiöse Gesundheitsgefahren zu beurteilen. Dadurch unterstützt der Ansatz europäische Länder dabei, die neuen Kernanforderungen zur Surveillancekapazität der Internationalen Gesundheitsvorschriften (2005) einzuhalten. In der Dissertation wurde ein Syndromsurveillancesystemkonzept vorgestellt, das durch das Ausbalancieren von Harmonisierungsbemühungen und Diversität in den europäischen Kontext passt. Zudem wurden in der Dissertation Schlüsselerfolgsfaktoren identifiziert, die in die Gestaltung von Syndromsurveillancesystemen einfließen können.

Vor einer breiteren Implementierung von Syndromsurveillancesystemen war mehr Evidenz zum Nutzen des Ansatzes notwendig - über einzelne Fallstudien hinaus. Die Dissertation hat, basierend auf dem Vergleich verschiedener Syndromsurveillancesysteme und verschiedener Länder, solche Evidenz geliefert. Das einzigartige europäische ,Labor' hat diese Art vergleichende Forschung und das Generieren neuen Wissens für Folgeaktivitäten ermöglicht. 

Acknowledgements 
The journey of becoming a Doctor was as exiting and instructive as I hoped it would be - I would not want to have missed it for the world! I would like to thank my supervisors, Helmut Brand and Thomas Krafft, for enabling me to go on this journey and accompanying me all the way. Dear Helmut, already at the very beginning, before joining the Department of International Health as a researcher, I remember travelling back to Germany from our meetings in Maastricht (fittingly with the 'euregiobahn'), being inspired by your vision and ideas, which made me eagerly wanting to become a part of the European Public Health community. Thank you for being there for me with a signpost full of possible directions whenever I could not see the way ahead!

Dear Thomas, we have been travelling together for more than 13 years now and I am truly grateful for all the new worlds you opened to me. Thank you for the Foro Romano, the Old Trafford, the Deogarh Mahal, the Great Lakes, and the Jean-Monnet Building. Thank you for DFG-Schnittchen, Finnish potatoes, and Chinese I-have-absolutely-noidea-what-it-was. Thank you for medical geography, health information and emergency care, for global changes and healthy cities, for strategic thinking and the depths of project management. I would not be anywhere near where I find myself now without you!

Thank you very much Jouke van der Zee, Kasia Czabanowska, Anette Hulth, Thomas Kistemann, and David Townend, for agreeing to review my work as a member of my assessment and supervisory committee and for taking the effort to also join the defence!

This dissertation would not have been possible without the two Health Programme Actions SIDARTHa and Triple S-AGE. Many thanks for the support, expertise and company of the people involved in these two actions, especially the coordinators, Luis Garcia-Castrillo Riesgo and Anne Fouillet and the members of the respective steering committees. Thank you for more than six great years in the European project world!

I especially owe my co-authors who were either also involved in the SIDARTHa or Triple S-AGE action or in the subsequent work for the articles included in this thesis: Helmut Brand, Alex Elliot, Matthias Fischer, Anne Fouillet, Luis Garcia-Castrillo Riesgo, Loic Josseran, Alexander Krämer, Thomas Krafft, Freddy Lippert, Javier Llorca, Jim McMenamin, Daniela Popa, Gillian Smith, Marta Sala Soler, Gernot Vergeiner, and AnneCatherine Viso. Thank you for the good collaboration, fruitful discussions and support in all stages from idea to published article! My special thanks go to Boris Kauhl, Nicole Rosenkötter and Sabrina Schrell for their invaluable input to the publications included as chapters 3, 4 and 5 in this thesis! I would also like to thank the reviewers of the published articles included in this thesis from whom I have really learned a lot.

Through the time of my PhD, I had great colleagues who accompanied and supported me in so many ways - professionally and personal. My big thanks to those who created the unreproducable GEOMED-time, to my colleagues at INTHEALTH and especially my fellow $\mathrm{PhD}$ candidates for sharing most of the PhD-path with me - step by step, article by article, experience by experience; and to the Team of Acute Zorg Euregio for their support on the last mile!

Tremendously more difficult, much less instructive and far more boring would those last years have been without you, Nicole. Since 2010, I am privileged to call you my colleague, co-author, flatmate, mirror, inspiration and very good friend. Thank you for all kitchen table talks, email exchanges, Skype calls, joint travels, beers and pasta and for standing by my side on the last day of my PhD journey. To many joint years to come! 
Thank you so much, Tine, for becoming my paranymph, for always being there - even over large distances in space and time - since we were six years old, for talks in a language only we can understand, for convincing me that acting, Bon Jovi, taking a night train to Paris or getting red hair (...among many other things) would be valuable experiences (and for being right with that). To life-long friendship which is the most indestructible!

Last but not least, a big thank you to my family and friends who supported me one way or the other, may that have been by designing and printing this book, by spending valuable time-off with me when it was most needed, by patiently waiting weeks and months for me to return calls and by celebrating this milestone with me! Especially, I would like to thank my Mom for letting me chase my dreams while supporting me every second, for leaving me alone and for nudging me at the right times, for being the best role model while letting me become who I am, for every advice from how to make my favourite dishes to dealing with life crises. You ground me and give me wings! 

Curriculum Vitae 


\section{Curriculum Vitae}

Alexandra Ziemann was born on 15 September 1979 in Osterholz-Scharmbeck, Germany. She graduated from Gymnasium Osterholz-Scharmbeck in 1999. In the same year, she pursued her studies of geography, history, public and international law, and public health at the universities of Greifswald, Bonn and Munich, Germany. In 2006, she graduated from Munich University with a diploma in human geography. Her diploma thesis dealt with the implementation of the healthy settings approach in Delhi, India. During her studies, Alexandra Ziemann worked for four years as student assistant and freelancer at the Scientific Secretariat of the German National Committee on Global Change Research and GEOMED Research Group. Her work focused on emergency medical services research, health geography, environmental and public health. At this time, she also got involved in her first European Commission co-funded action, the European Emergency Data Project, which explored the use of routinely collected emergency care data for public health monitoring. After graduation, she worked for one year as researcher and lecturer in Geography at Cologne University, Germany, before joining a spin-off of the GEOMED Research Group. From 2007 until 2010, Alexandra Ziemann worked as researcher and authorised representative at GEOMED Research Forschungsgesellschaft $\mathrm{mbH}$ in Bad Honnef, Germany. During this time, she worked as project officer on the European Commission co-funded SIDARTHa action, which aimed at developing an emergency databased syndromic surveillance system. From 2010 until 2014, she worked as researcher at the Department of International Health, CAPHRI School of Public Health and Primary Care, Maastricht University, The Netherlands. During that time, she coordinated a work package as part of the European Commission co-funded action Triple S-AGE focusing on inventorying and harmonising syndromic surveillance in Europe. Since 2014, Alexandra Ziemann works as policy advisor on regional cross-border emergency care between Germany and the Netherlands at Bureau Acute Zorg Euregio in Enschede, The Netherlands, where she is also involved in emergency medical services research. In 2014, she won a Young Forum Gastein Scholarship, which was awarded by the International Forum Gastein and the European Commission. In 2015, she served as temporary consultant to the World Health Organization Regional Office for Europe on evidenceinformed health policy.

\section{Publications}

Ziemann A, Fouillet A, Brand H, Krafft T. Success factors of European syndromic surveillance systems: A Qualitative Comparative Analysis. [Submitted]

Ziemann A, Rosenkötter N, Garcia-Castrillo Riesgo L, Fischer M, Krämer A, Lippert FK, Vergeiner G, Brand $H$, Krafft T. Meeting the International Health Regulations (2005) surveillance core capacity requirements at the subnational level in Europe: The added value of syndromic surveillance. BMC Public Health 2015;15:107.

Ziemann A, Rosenkötter N, Garcia-Castrillo Riesgo L, Schrell S, Kauhl B, Vergeiner G, Fischer M, Lippert FK, Krämer A, Brand H, Krafft T. A concept for routine emergency-care data-based syndromic surveillance in Europe. Epidemiology and Infection $2014 ; 142: 2433-2446$. 
van Asten L, Fanoy EB, Hooiveld M, Koopmans MP, Kretzschmar ME, Enserink R, de Jong S, Koppeschaar C, Reusken C, van Steenbergen JE, Veldman-Ariesen MJ, van Wuyckhuise LA, Ziemann A, van den Wijngaard CC, Bijkerk P. Syndroomsurveillance: een vinger aan de pols van de volksgezondheid [Syndromic surveillance: a finger on the pulse of public health]. Nederlands Tijdschrift voor Geneeskunde 2014;158(0):A7415. Dutch.

Rosenkötter N, Ziemann A, Krafft T, Garcia-Castrillo Riesgo L, Vergeiner G, Brand H. Noninfectious event detection under the International Health Regulations (2005) in Europe a case for syndromic surveillance. Journal of Public Health Policy 2014;35(3):311-26.

Krafft T, Ziemann A. Notfallmedizin und öffentliche Gesundheit - Einsatz notfallmedizinischer Daten für Fragen der öffentlichen Gesundheit in Europa [Emergency medicine and public health - Application of emergency data for public health questions in Europe]. In: Neumayr A, Schinnerl A, Baubin M, editors. Qualitätsmanagement in der prähospitalen Notfallmedizin. Heidelberg: Springer; 2013. p. 31-42. German.

Rosenkötter N, Ziemann A, Garcia-Castrillo Riesgo L, Gillet JB, Vergeiner G, Krafft T, Brand $\mathrm{H}$, for the SIDARTHa project consortium. Validity and timeliness of syndromic influenza surveillance during the autumn/winter wave of $A(H 1 N 1)$ influenza 2009. Results of emergency medical dispatch, out-o f-hospital emergency physician service and emergency department data from three European regions. BMC Public Health 2013;13(1):905.

Schrell S, Ziemann A, Garcia Castrillo-Riesgo L, Rosenkötter N, Llorca, J, Krafft T. Local implementation of a syndromic influenza surveillance system using emergency department data in Santander, Spain. Journal of Public Health (Oxford) 2013;35(3):397403.

Fischer M, Kamp J, Garcia-Castrillo Riesgo L, Robertson-Steel I, Overton J, Ziemann A, Krafft T; EED Group. Comparing emergency medical service systems - a project of the European Emergency Data (EED) Project. Resuscitation 2011;82(3):285-93.

Sala Soler M, Fouillet A, Viso AC, Josseran L, Smith GE, Elliot AJ, McMenamin J, Ziemann A, Krafft T. Triple S Project. Assessment of syndromic surveillance in Europe. Lancet 2011, Nov 26;378(9806):1833-4.

Ziemann A, Krafft T, Rosenkötter N, Garcia-Castrillo Riesgo L, Vergeiner G, Fischer M, Lippert F, Krämer A, Pinheiro P, Brand H, Rosenberg M, Overton J. Syndromic Surveillance: Enhancing Public Health Responsiveness to Global Change - A European Perspective. IHDP Update $2011 ; 1: 12-18$.

Krafft T, Ziemann A, Tenelsen T, Garcia-Castrillo Riesgo L, Fischer M, Krämer A, Lippert F, Vergeiner G. Raum-zeitliche Analysealgorithmen zur Früherkennung von Gesundheitsgefahren - Der SIDARTHa-Ansatz [Spatio-temporal analysis algorithms for early detection of health threats - The SIDARTHa approach]. In: Strobl J, Blaschke T, Griesebner G, editors. Angewandte Geoinformatik 2009. Beiträge zum 21. AGITSymposium Salzburg; 2009. p. 238-243. German. 


\section{Presentations}

Ziemann A, Fouillet A, Sala Soler M, Krafft T. Review of morbidity syndromic surveillance activities: Use and usefulness of different syndromic data sources in Europe and around the world. European Public Health Conference, Brussels/Belgium, 13-16 November 2013.

Ziemann A, Krafft T, Fouillet A, Garcia-Castrillo Riesgo L. Syndromic surveillance in Europe. 18th World Congress on Disaster and Emergency Medicine, Manchester/United Kingdom, 28-31 May 2013.

Ziemann A, Krafft T, Sala Soler M, Fouillet A, Hulth A, Müller L, Conti S, Kanieff M, Dupuy $C$, Medina $S$, the Triple-S project consortium. Use of different data sources for syndromic surveillance in Europe. International Meeting on Emerging Diseases and Surveillance 2013, Vienna/Austria, 15-18 February 2013.

Ziemann A, Krafft T, Brand H, Sala Soler M, Fouillet A, Hulth A, Müller L, Molbak K, Conti S, Kanieff M, Rago G, Perrin JB, Dupuy C, Medina S, the Triple-S project consortium. Identifying good practice for syndromic surveillance in Europe - A comparative study based on site visits in eight countries. European Public Health Conference, St. Julians/Malta, 7-10 November 2012.

Ziemann A, Garcia-Castrillo Riesgo L, Kauhl B, Schrell S, Rosenkötter N, Fischer M, Vergeiner G, Gillet JB, Meulemans A, Krafft T. Added value of routine emergency medical data for detecting clusters of acute gastrointestinal illness in Europe. European Resucitation Congress, Vienna/Austria, 18-20 October 2012.

Ziemann A, Pinheiro P, Rosenkoetter N, Garcia-Castrillo Riesgo L, Krafft T, Kraemer A, Fischer M, Gillet JB, Meulemans A, Vergeiner G, Lippert F, Brand H, the SIDARTHa project consortium. Evaluation of the European syndromic surveillance approach SIDARTHa. European Public Health Conference, Copenhagen/Denmark, 9-12 November 2011.

Ziemann A, Krafft T, the SIDARTHa project consortium. Spatial-temporal syndromic surveillance in Europe: results from the SIDARTHa project. International Medical Geography Symposium, Durham/United Kingdom, 10-15 July 2011.

Ziemann A, Krafft T, for the SIDARTHa project consortium. Syndromic Surveillance I: A European Approach to Enhance Early Warning and Outbreak Detection. 10th International Seminar on Medical Geography, Rome/Italy, 19 December 2010.

Ziemann A, Krafft T, Garcia-Castrillo Riesgo L, Fischer M, Kraemer A, Lippert F, Vergeiner G. The SIDARTHa approach - European emergency data-based syndromic surveillance. European Public Health Conference, Lodz/Poland, 25-28 November 2009.

Ziemann A, Krafft T, Garcia-Castrillo Riesgo L, Fischer M, Kraemer A, Lippert F, Vergeiner $G$, on behalf of the SIDARTHa consortium. A European syndromic surveillance approach based on routine emergency care data - First results from the SIDARTHa project. International Meeting on Emerging Diseases and Surveillance, Vienna/Austria, 14 February 2009.

Ziemann A, Krafft T, Garcia Castrillo Riesgo L, Fischer M, Krämer A, Lippert F, Vergeiner $G$, for the SIDARTHa project group. "Syndromic Surveillance" - State-of-the-Art und Umsetzungsmöglichkeiten in Europa ["Syndromic surveillance" - State-of-the-art and application options in Europe]. Annual Meeting of the German Society of Social Medicine and Prevention, Hannover/Germany, 17-19 Sepbember 2008. German. 